\title{
Past, present and future of the Biginelli reaction: a critical perspective
}

\author{
Suresh and Jagir S. Sandhu* \\ Department of Chemistry, Punjabi University, Patiala-147 002, Punjab, India \\ E-mail:__sandhu2002@yahoo.com
}

\begin{abstract}
This review covers up to 2010 and some available references of 2011 of synthetic advances in the Biginelli reaction, including recent mechanistic advances, new building blocks, new pharmacological disclosures and asymmetric syntheses. Also present account is covering all aspects of the reaction whereas some of previous ones emphasized one aspect and others had passing reference.
\end{abstract}

Keywords: Biginelli reaction, multicomponent reactions, green process

\section{Table of Contents}

1. Introduction

2. Discovery

3. Mechanism

4. Pharmacology

4.1 Antihypertensive agents

4.2 Potassium channel antagonists

4.3 Anti-HIV agents

4.4 Antitumor activity

4.5 Anti-epileptics

4.6 Anti-malarials

4.7 Anti-microbials

4.8 Anti-inflammatories

4.9 Anti-tubercular activity

4.10 Anti-bacterial activity

4.11 Miscellaneous activities

5. Scope of reaction; developments in structural variants

5.1 Aldehydes 
5.2 Urea

5.3 Active hydrogen components

5.4 Use of alcohols in place of aldehydes

6. Catalyst variations

6.1 Brønsted acids

6.2 Lewis acids

6.3 Ionic liquids

6.4 Biocatalysts

6.5 Organocatalysts

7. Rate enhancements

7.1 Sonication

7.2 Microwave irradiation

7.3 Miscellaneous processes

8. Biginelli scaffold variations and Biginelli like reactions

9. Asymmetric Biginelli reaction

10. Conclusions; future outlook

11. Acknowledgements

12. References

\section{Introduction}

Hantzsch, ${ }^{1}$ Knoevenagel, ${ }^{2}$ and Biginelli reactions, ${ }^{3}$ have some similarity; as each one of these employs aldehyde, acetoacetic ester (active methylene compound). The earliest of these seems to be the discovery of the Hantzsch reaction which was reported in 1881, ${ }^{1}$ wherein Hantzsch heated acetoacetic ester, an ammonia source, and an aldehyde, to obtain the now well-known dihydropyridines or Hantzsch pyridines 1. A decade later the Italian chemist P. Biginelli, ${ }^{3}$ reacted same two components in equimolar ratio viz. acetoacetic ester, aldehyde and third component as urea in acidic alcoholic solution to obtain a new compound, the now well-known 3,4-dihydropyrimidin-2(1H)-ones or Biginelli compounds, ${ }^{4} 2$ which are obvious aza-analogues of the Hantzsch dihydropyridines. Biginelli did not detect any Hantzsch dihydropyridines $\mathbf{1}$ as byproducts. 5 
<smiles>CCOC(=O)C1=C(C)NC(C)=C(C(=O)OCC)C1c1ccccc1</smiles>

1

Hantzsch pyridines

(Dihydropyridine)<smiles>CCOC(=O)C1=C(C)NC(=O)NC1c1ccccc1</smiles>

2

Biginelli compound

(Dihydropyrimidine)

He apparently did this reaction in a multicomponent way, and currently the development of multicomponent reactions (MCRs) is an integral part of numerous research efforts around the world involved in the drug development programs to achieve synthetic targets in expeditious way. It seems old discoveries are new fashions of the present times. Subsequent investigators have tried to understand the course of reaction and they invoke the participation of the Knoevenagel reaction.

In the initial years there was not much synthetic activity in this reaction but during last 100 years or so this reaction received much attention and as a result there were nearly five hundred research publications, mostly involving catalyst changes. During these years from its discovery emphasis was on understanding the course of reaction, with some emphasis on structural variants. Subsequent to these academic developments the Biginelli scaffold was shown to be of great value from a pharmaceutical point of view; because of this importance, investigations were very fast, and virtually every major journal was flooded with papers on the Biginelli reaction. ${ }^{6}$ Major emphasis being on process streamlining mainly Lewis acid catalyst etc. In this account, update on catalyst variations, asymmetric synthesis, scaffold variations and Biginelli like reactions aspect of this reaction is presented. During the past decade or so publications have been so fast and numerous, some of these may get missed incidently so authors feel sorry for that if it happens.

\section{Discovery}

Historically, Italian chemist Pietro Biginelli (University of Florence) reported this reaction for the first time which is taken as the birth of this reaction; it is popularly named after him i.e. Biginelli Reaction. ${ }^{3}$ Classically, he did it as acid-catalyzed condensation of ethyl acetoacetate, benzaldehyde, and urea in ethanol by refluxing the mixture and on cooling he obtained a solid crystalline product 3,4-dihydropyrimidin-2(1H)-one which apparently was a three component reaction (Scheme 1), the acid used here was hydrochloric acid. Though it is more than 100 years acidic catalysts continue to be used though the number of variation done in this catalysts system 
run into hundreds so is the case of solvent systems and several heating mode changes have been carried in a flood of research publications in the following pages these developments shall be discussed and catalysts used changes made are from mild Bronsted acids to strong Lewis acid what so ever was at hand rather it raise questions is catalyst really needed. ${ }^{6(1)}$

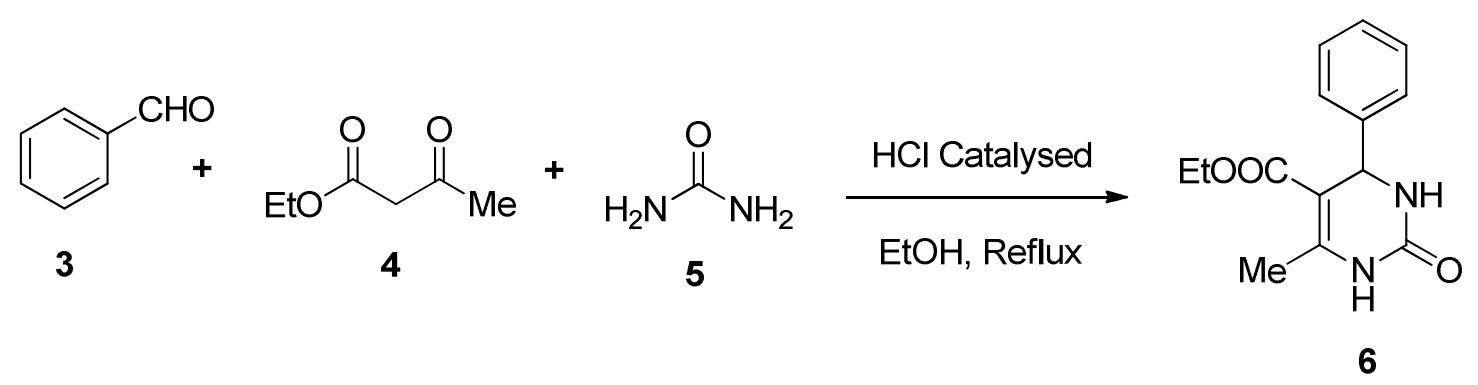

Scheme 1. Classical synthesis of Biginelli product.

The most attractive part for this motif is biological activity and asymmetric synthesis of compounds which will be discussed in separate sections. When one goes through all this research activity other than asymmetric synthesis or the motif modification remaining seems to be of academic interest only. These two, namely asymmetric synthesis and motif modifications required a good amount of effort, good chemistry and also are of applied in nature since this may lead to some applications.

\section{Mechanism}

As a normal curiosity of chemists after the discovery/observation of a reaction there were studies to find the expected pathway followed by this reaction some possibilities are as under. As Biginelli reaction involves condensation of three component aldehydes 3, 1,3-carbonyl compounds 4 and (thio)urea $\mathbf{5}$, keeping these reactants reactivity in mind plausibly the reaction could proceed in following ways.

1. Condensation of aldehyde $\mathbf{3}$ with 1,3-carbonyl compounds $\mathbf{4}$ via aldol condensation followed by nucleophilic attack of urea 5 molecule.

2. Condensation of aldehyde $\mathbf{3}$ with 1,3-carbonyl compounds $\mathbf{4}$ via Knoevenagel subsequently nucleophilic addition of urea 5.

3. Condensation of aldehyde $\mathbf{3}$ with urea molecule $\mathbf{5}$ (via N-benzylidene-urea) and then nucleophilic addition of 1,3-carbonyl compound 4.

4. Condensation of aldehyde $\mathbf{3}$ with two urea molecules 5 (via N,N-benzylidenebisurea) \& further nucleophilic addition of 1,3-carbonyl compound 4.

5. Nucleophilic condensation of urea $\mathbf{5}$ on 1,3-carbony compound $\mathbf{4}$ (via 3-ureido-crotonates) and after that again nucleophilic attack of this condensate to aldehyde $\mathbf{3}$. 


\section{Folkers and Johnson (1933)}

The first attempt made to understand the correct pathway of this reaction was by Folkers et al. in 1933. ${ }^{7}$ Under acidic conditions they could prove an intermediate 1,1'-(phenylmethanediyl)diurea 7 to transform to end product Biginelli compound (as shown in route 4); they proposed the intermediacy of $\mathbf{9}$ and $\mathbf{1 0}$ (see scheme 2).<smiles>NC(=O)NC(NC(N)=O)c1ccccc1</smiles>

7<smiles>CCOC(=O)/C(=C/c1ccccc1)C(C)=O</smiles>

9<smiles>CCOC(=O)/C=C(\C)NC(N)=O</smiles>

10

Scheme 2. Intermediate proposed by Folkers and Johnson.

\section{Sweet and Fissekis (1973)}

After several decades the reaction was reinvestigated by Sweet and Fissekis ${ }^{8}$ who advocated contradictory mechanism to Folkers suggestion as indicated above in route 1, proceeding through aldol reaction (through carbenium ion intermediate 8) (Scheme 3).

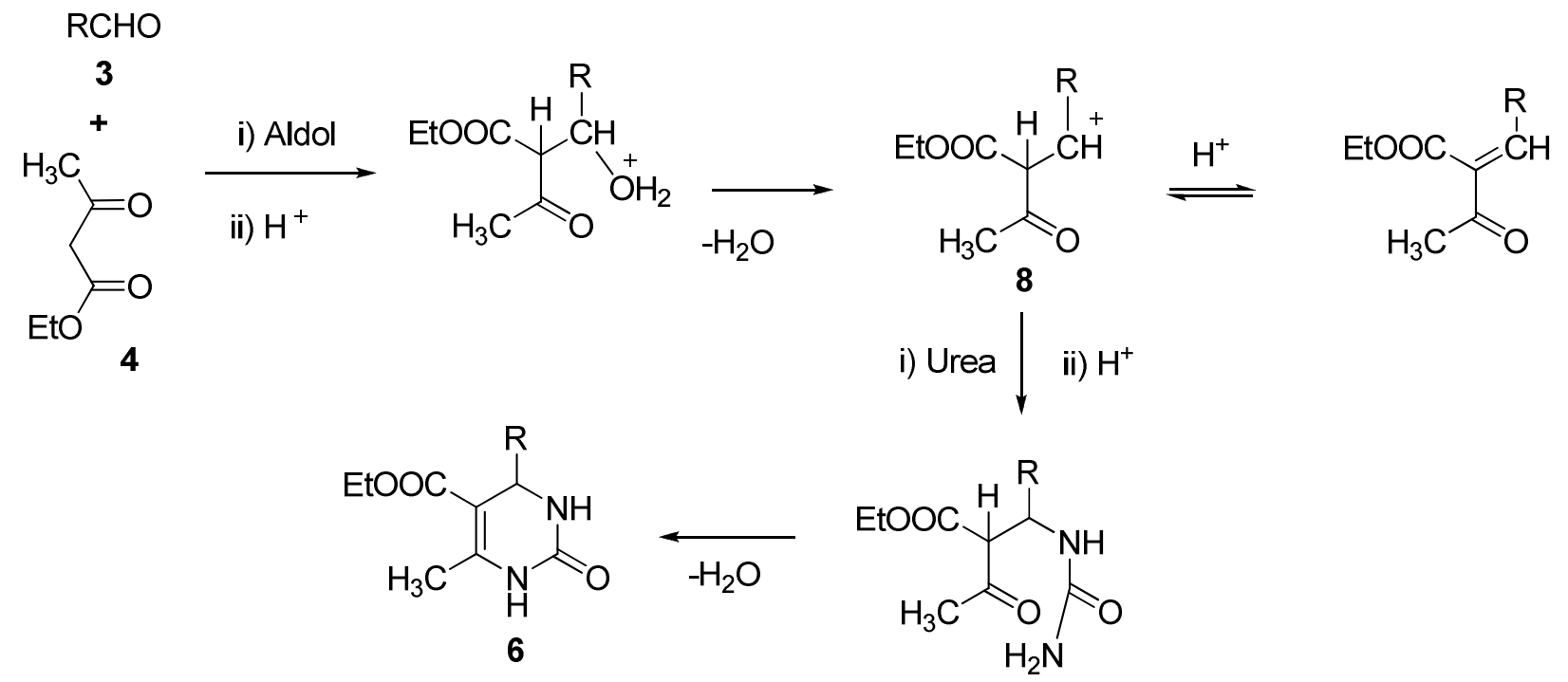

Scheme 3. Formation of 3,4-dihydropyrimidinone via aldol condensation.

\section{Atwal and O'Reilly (a two step process) (1987)}

Atwal and his associates, ${ }^{9-11}$ gave a proposal to surmount troubles linked with poor yield of the typical Biginelli compounds mainly in the case of aliphatic aldehydes and aldehydes having a slightly hindered carbonyl function by ortho-substituents. This new approach involving two steps, 
first step concerned with separate synthesis of unsaturated carbonyl compound $\mathbf{9}$ via Knoevenagel condensation and second step involved the base catalyzed addition of substituted ureas as shown in Scheme 4, as in route 2 .

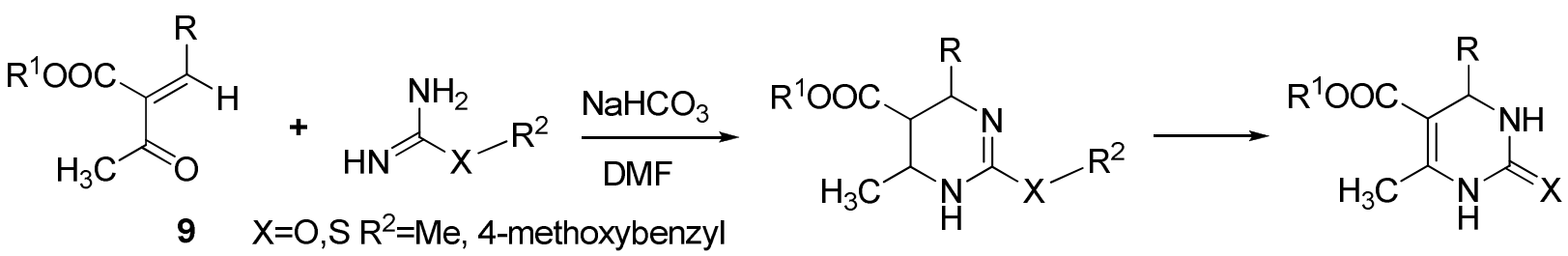

Scheme 4. Formation of 3,4-dihydropyrimidinone via Knoevenagel condensation.

It is pertinent to mention here that this modification of the Biginelli reaction has rarely been used in recent years, since it involves two steps.

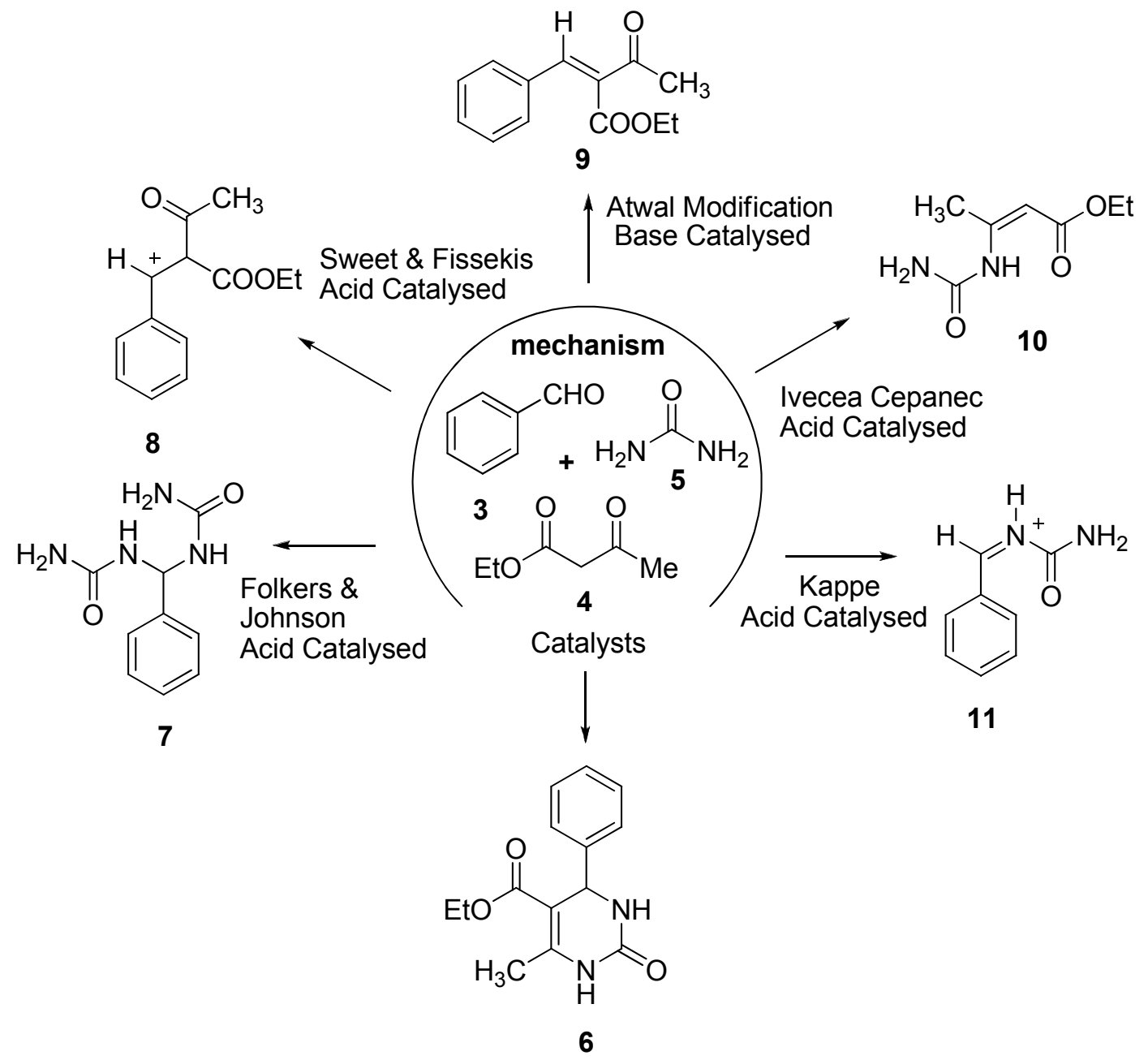

Scheme 5. Investigated intermediates by various workers. 
Depicting of all these mechanistic proposals so far advanced were necessary to be presented here because there are very few synthetic studies on mechanism aspect of this reaction prior to or after Kappe's mechanistic proposal though this reaction has attracted the attention of large number of chemists around the world as far as number of publications are concerned clearly these were catalysts efficacy/ catalyst development investigations only. It is not out of point to mention here every research journal was flooded describing these changes i.e. catalysts and every paper made a mention of Kappe's mechanism which was based upon spectroscopic evidence only and is given below.

\section{O. Kappe (1997)}

On the basis of spectral techniques like $\mathrm{H}^{1} / \mathrm{C}^{13}$ NMR spectroscopy, Kappe, ${ }^{12}$ further reexaminated the mechanism of this multicomponent reaction. In this proposed mechanism, the first step involved nucleophilic attack of urea on the electron deficient carbon of the aldehyde function under acidic conditions results formation of $\mathrm{N}$-acyliminium ion intermediate 11 takes place at the expense of acid catalysed dehydration. In the next step, active methylene compound adds onto this intermediate in a Michael fashion as in route 3. He found that in this reaction dihydropyridines were always formed in minor quantities, ${ }^{6}$ which had not been observed by earlier research groups (Scheme 2).

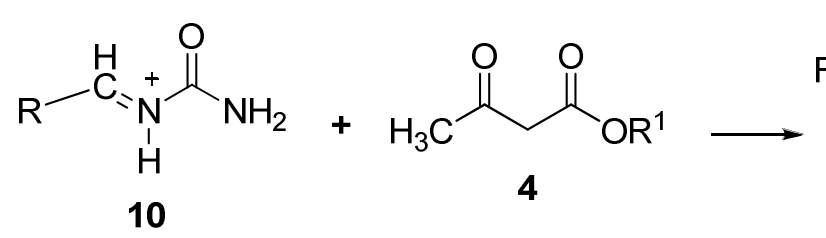<smiles>[R20]OC(C(C)=O)C([R])NC(N)=O</smiles>

12<smiles>[R20]OC1C([R])NC(=O)NC1(C)O</smiles>

13

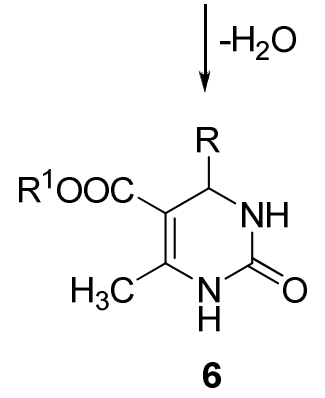

Scheme 6. Formation of 3,4-dihydropyrimidinone via $N$-acyliminium ion.

When Saloutina and co-workers ${ }^{13}$ used $\mathrm{CF}_{3} \mathrm{COCH}_{2} \mathrm{CO}_{2} \mathrm{Et}$ in place of acetoacetic ester they isolated the intermediate $\mathbf{1 3}$ and the dehydration is done in the next step using $p$-toluenesulfonic acid. Using $\mathrm{GaCl}_{3}$ as Lewis acid the dehydration part in this mechanism was also investigated by us. ${ }^{14} \mathrm{We}$ observed that anhydrous $\mathrm{GaCl}_{3}$ yields final products in excellent yields in contrast to hydrated $\mathrm{GaCl}_{3}{ }^{15}$ which does not perform well in this reaction. 


\section{Cepanec (experimental evidence) 2007}

Using antimony trichloride, ${ }^{16}$ a typical Lewis acid catalyst reaction mechanism was in real terms studied rather it was found under these conditions that reaction proceeds via intermediate $\mathbf{1 0}$ and not iminium formation as proposed by Kappe authors are reproducing actual scheme see below:
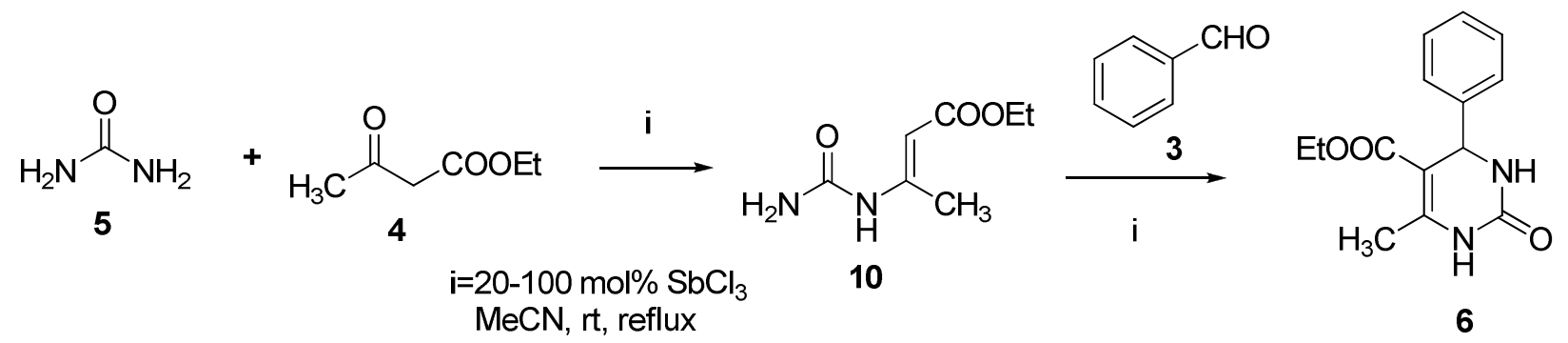

Scheme 7. Formation of 3,4-dihydropyrimidinone via 3-ureido-crotonates.

This Lewis acid behaviour places questions on several reports describing the Biginelli reaction and proposing Biginelli mechanism similar to Kappe without working out actual details.

\section{Jian-Hua Zhou (DFT Study) 2008}

DFT study is reported via condensation of benzaldehyde, urea, and ethyl acetoacetate is investigated under classical reaction conditions and these authors ${ }^{17}$ also confirmed Kappe's proposal.

\section{De Souza 2009}

Lately of course there are mechanistic investigations De Souza et al. ${ }^{18}$ investigated Biginelli reaction and concluded in favour of Folkers and Johnson proposal, they based their conclusions on density functional theory calculations (DFT), they have used a mass spectrometer having accessories for various ionizations: for details see reference 18 .

Under acidic condition (formic acid was used) found their experimental and theoretical investigations regarding reaction the mechanism in favor intermediate $\mathrm{N}, \mathrm{N}$-benzylidenebisurea 7 as proposed by Folkers and Johnson. They examined the reaction pathway using direct infusion electrospray ionization mass spectrometry (ESI-MS) and density functional theory (DFT). In this way, these workers support the formation of $\mathbf{7}$ with traces of intermediate 9 and 10. As mentioned by us chase for easy publications is slowing down and trend is there for actual path determinations. So another research group Boumoud et al. also reported their investigation using catalyst nickel(II) nitrate hexahydrate and they concluded in favour of Folkers mechanism. 
<smiles>CCOC(=O)/C=C(/C)OC</smiles><smiles>CCOC(=O)C1=C(C)NC(=O)NC1c1ccccc1</smiles><smiles>CCOC(=O)C(NC(N)=O)C(C)=O</smiles><smiles>CCOC(=O)C(C(C)=O)C(NC(N)=O)c1ccccc1</smiles>

6

Scheme 8. Formation of 3,4-dihydropyrimidinone via iminium mechanism 11.

\section{Shun-Jun Ji 2010 (base catalysed)}

A large number of investigations have been on the use Lewis acid acid like catalysts, and only very few papers describe basic catalysts. Very recently, Chinese workers, ${ }^{19}$ have described the use of strong bases and proposed different pathways as shown below.<smiles>[R]C1=C([R])C([R])NC(=O)N1</smiles><smiles>[R]C(NC(N)=O)NC(N)=O</smiles><smiles>[R]CC([R])=O</smiles>

$\mathrm{i}=t-\mathrm{BuOK}(20 \mathrm{~mol} \%), \mathrm{EtOH}, 70^{\circ} \mathrm{C}$

Scheme 9. Base catalysed synthesis of 3,4-dihydropyrimidinone.

All the above mentioned processes and mechanism reinvestigations with each catalysts used seems to be an healthy trend in this reaction in contrast to earlier reports casually mentioning that our reaction seems to follow Kappe mechanism. In mechanism advancements yet another mechanism report is there using hexaaquo- $\mathrm{Al}(\mathrm{III}) \mathrm{BF}_{4}{ }^{20}$ 


\section{Litvic 2010 (Brønsted acid)}

The mechanism of this reaction reported to via 'ureido-crotonate' formation $\mathbf{1 0}$ as reported by Cepanec in contrast to acylimino intermediate 11 (Kappe suggestion). ${ }^{20}$

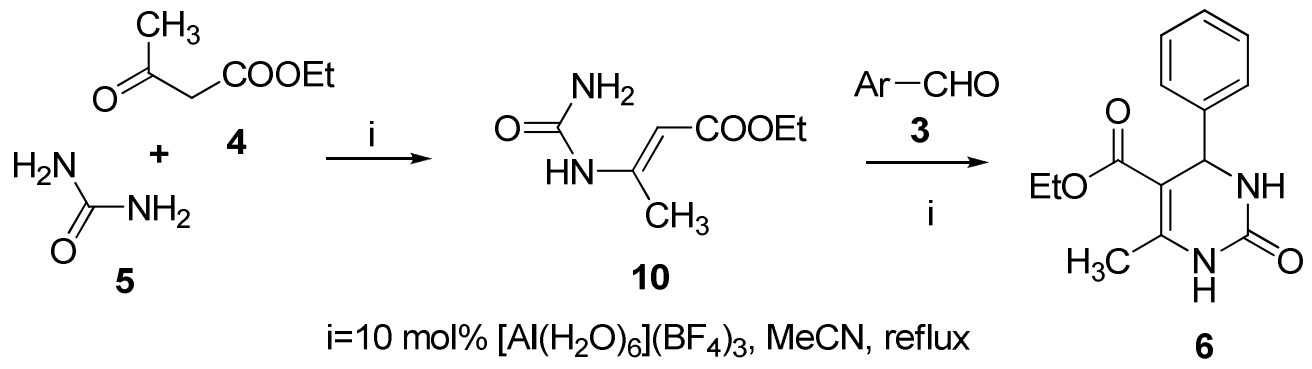

Scheme 10. Acid catalysed synthesis of 3,4-dihydropyrimidinone.

\section{Pharmacology}

In this part of this account biological aspects of this motif are discussed. A biologist needs readily available, stable molecules for evaluation/study which this scaffold fulfills. In 1930, wool protection activity of these molecules was patented. ${ }^{21}$ There followed further intensive investigation because of their resemblance to clinically used nifedipine 14-16 Biginelli being their aza-analogue 17-18 further they had resemblance to marine natural alkaloids batzelladine B 19 (for this complete comparison see below). ${ }^{22,23}$

Variation of all three building blocks, viz. active methylene, ureas, aldehyde component lead to extension of the scope original multi-component resulting in large molecular diversity of dihydropyrimidines. The biological investigation of these various molecules via molecular manipulation showed activities like antiproliferative, antiviral, antitumor, anti-inflammatory, antibacterial, antifungal, and antitubercular activity. Similarly, the structural core of quinoline is frequently associated with medicinal applications such as anticancer, antimicrobial, HIV-1 integrase inhibition, HIV protease inhibitors, antileishmanial activity, NK-3 receptor antagonists, PLT antagonists, and antimalarial activity.

In search of more potent and effective medicinal important molecules numerous Biginelli dihydropyrimidine related annulated or multifunctionallized pyrimidines heterocyclic have been investigated or tested against different dangerous diseases which is arise due to stress or pollution. It is worth mentioning here that these new dihydropyrimidines are synthesized in classical fashion or employing different reaction condition which are discussed in the catalyst section. In the following paragraphs only selective molecules are presented which are have significant activity and they are examined with clinically used drugs in vivo/in vitro and establishing QSAR. 
<smiles>CCOC(=O)C1=C(C)NC(C)=C(C(C)=O)C1c1cccc(Cl)c1Cl</smiles>

Felodipine

14<smiles>CC1=C(C(=O)OC(C)C)C(c2cccc([N+](=O)[O-])c2)N(C(N)=O)C(=O)N1</smiles>

17<smiles>COC(=O)C1=C(C)NC(C)=C(C(OC)OC)C1c1ccccc1[N+](=O)[O-]</smiles>

15

16<smiles>CC1=C(C(=O)OC(C)C)C(c2ccccc2C(F)(F)F)N(C(=O)OC2CCN(Cc3ccc(F)cc3)CC2)C(=S)N1</smiles>

18

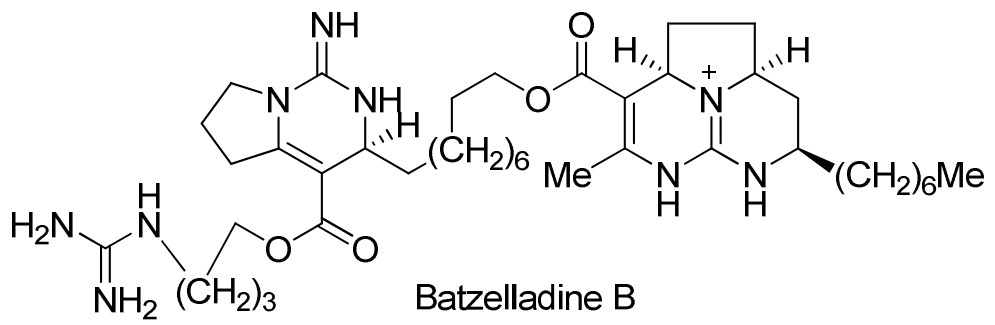

19

\subsection{Antihypertensive agents}

As a usual temptation biologists saw Biginelli products resemblance to Hantzsch 1,4dihydropyridine (indeed these were side products in original experiment) as being aza-analogues of nifedipine and other related molecules which are well-known calcium channel modulators and Biginelli compounds viz SQ 32926 17, SQ 3254718 (effective orally active antihypertensive agents) are promising targets for bringing them to actual use. Hetero-substituted DHPMs 20 with a branched ester (e.g. isopropyl, sec-butyl) and an alkylthio group (e.g. SMe) was found to be optimal for biological activity. In these compound $\mathbf{2 1}$ is potent mimic of dihydropyridine calcium channel blockers. ${ }^{24}$ 
<smiles>[R2]OC(=O)C1=C(C)NC([R])=NC1c1cccc([R])c1</smiles>

$\mathrm{R}=2-\mathrm{NO}_{2}, 3-\mathrm{NO}_{2}, 2-\mathrm{CF}_{3}, 2,3-\mathrm{Cl}$.

$\mathrm{R}^{1}=\mathrm{Me}, \mathrm{CH}_{2} \mathrm{CH}=\mathrm{CH}_{2}, \mathrm{CH}_{2}\left(\mathrm{CH}_{2}\right)_{3} \mathrm{CH}_{3}$,

$\mathrm{CH}_{2} \mathrm{C}_{6} \mathrm{H}_{5}, \mathrm{CH}_{2} \mathrm{CH}_{2} \mathrm{~N}(\mathrm{Me}) \mathrm{Bn}, \mathrm{CH}_{2} \mathrm{CH}_{2} \mathrm{NMe}_{2}$.

$\mathrm{R}^{2}=\mathrm{Et}$, $i-\mathrm{Pr}, \mathrm{Me}, \mathrm{SBu}, \mathrm{CH}_{2} \mathrm{CH}_{2} \mathrm{~N}(\mathrm{Me}) \mathrm{Bn}$.

$\mathrm{X}=\mathrm{O}, \mathrm{S}$.

$20(20)$<smiles>CCOC(=O)C1=C(C)NC(SCc2ccccc2)=NC1c1cccc([N+](=O)[O-])c1</smiles>

21

Other significant molecules like 22-27 are under serious investigation.<smiles>[R]c1cccc(C2NC(=S)NC(CCCC)=C2C(=O)OC)c1</smiles>

$\mathrm{R}=2-\mathrm{Cl}$, 3-Cl, 4-Cl, 3-Br, 4-Br, $2-\mathrm{CH}_{3}, 3-\mathrm{CH}_{3}, 4-\mathrm{CH}_{3}, 2-\mathrm{OCH}_{3}$, 2- $\mathrm{NO}_{2}, 3-\mathrm{NO}_{2}, 2-\mathrm{F}, 3-\mathrm{F}, 2-\mathrm{OC}_{2} \mathrm{H}_{5}$, $2-\mathrm{OH}-5-\mathrm{Br}$.

$22(16)$<smiles>[R][R]COC</smiles>

24 (11)<smiles>CCOC(=O)C1=C(CC)NC(=S)NC1c1ccccc1OCC</smiles>

23<smiles>[R][R]c1cccc(CSC2=NC(c3cccc([R])c3)C(C(=O)Nc3ccc([R])cc3)=C(C)N2)c1</smiles>

$\mathrm{R}=\mathrm{F}, \mathrm{Br}, \mathrm{NO}_{2}$.

$\mathrm{R}^{1}=2,4-\mathrm{Cl}, 4-\mathrm{Br}, 3-\mathrm{NO}_{2}, 4-\mathrm{NO}_{2}$ $3,4-\mathrm{OCH}_{3}, 4-\mathrm{OCH}_{3}$. $\mathrm{R}^{2}=\mathrm{H}, 2-\mathrm{Cl}, 3-\mathrm{Cl}, 4-\mathrm{Cl}, 4-\mathrm{F}$.

25 
<smiles>CC1=C(C(=O)Nc2ccc3[nH]ncc3c2)C(c2ccc(F)cc2)NC(c2ccccc2)N1</smiles><smiles></smiles>

Numericals in brackets show the number of molecules derived from respective structure investigated.

\subsection{Potassium channel antagonists}

Annulation of benzimidazole ring 28 with this Biginelli showed potassium channel antagonists activity and these are at preclinical developments $\mathbf{2 8 - 3 1}$. $^{25}$<smiles>COCC1=C(c2nc3cc(F)ccc3n2C)C(c2ccc(Cl)c(Cl)c2)n2nccc2N1</smiles>

28<smiles>CC1=C(C(=O)Nc2ccccc2)C(c2ccc(Cl)c(Cl)c2)n2nccc2N1</smiles>

29<smiles>CCCC(CCC)NC(=O)C1=C(C)Nc2ccnn2C1c1ccc(Cl)c(Cl)c1</smiles>

30<smiles>CC1=C(C(=O)N2CCC[C@H]2c2ccc(F)cc2)[C@@H](c2ccc(Cl)c(Cl)c2)n2nccc2N1</smiles>

31

\subsection{Anti-HIV agents}

Batzelladine A 32 and B 19 derivatives of DHPMs obtained from marine natural source have promising anti HIV activity. These low molecular weight derivatives inhibit the binding of HIV gp-120 to $\mathrm{CD}_{4}$ cells. $^{22}$ 


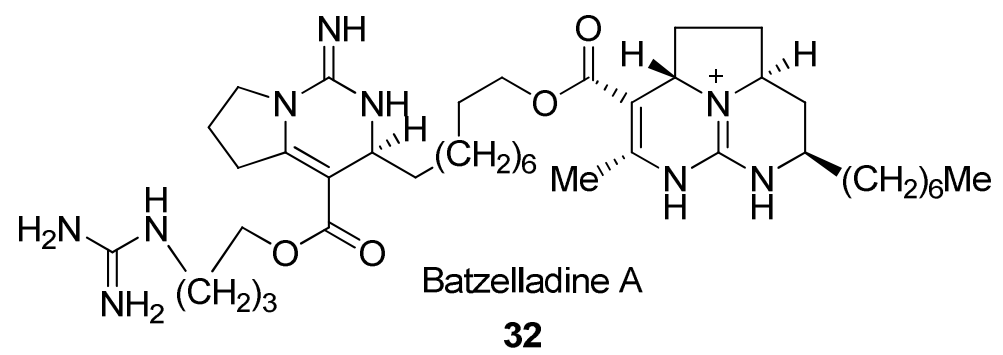

\subsection{Antitumor activity}

Human kinesin Eg5, an interesting drug target for the development of cancer chemotherapeutics. Monastrol 33 is the first Biginelli compound which has excellent anticancer activity, further a series of compounds for their ability to inhibit Eg5 activity has been investigated using two in vitro steady-state ATPase assays (basal and microtubule-stimulated) as well as a cell-based assay. In an attempt, another dihydropyrimidine i.e. furyl derivative $\mathbf{3 4}$ appeared more potent than monastrol by a fivefold factor. Reported compounds enastron 35, mon-97 36, dimethylenastron $\mathbf{3 5}$, and fluorastrol, ${ }^{26 \mathrm{~b}} \mathbf{3 6}$ potency of these new inhibitors, have been compared with the monastrol which are better fit of the ligand to the allosteric binding site and the addition of fluorine atoms. ${ }^{26}$<smiles>CCOC(=O)C1=C(C)NC(=S)N[C@H]1c1cccc(O)c1</smiles>

33<smiles>CC1=C(C(=O)c2ccco2)C(c2cccc(O)c2)NC(=S)N1</smiles>

34<smiles>[R]C1([R])CC(=O)C2=C(C1)NC(=S)N[C@H]2c1cccc(O)c1</smiles>

$\mathrm{R}=\mathrm{H}$ : (S)-enastron

$\mathrm{R}=\mathrm{Me}$ : (S)-dimethylenastron

35<smiles>[R]c1ccc(C(=O)C2=C(C)N(C)C(=S)NC2c2cccc(O)c2)cc1[R]</smiles>

$\mathrm{R}=\mathrm{R}^{1}=\mathrm{H}:(\mathrm{R})-\mathrm{mon}-97$.

$\mathrm{R}=\mathrm{R}^{1}=\mathrm{F}$ : (R)-fluorastrol.

$\mathrm{R}=\mathrm{H}, \mathrm{R}^{1}=\mathrm{F}$ : rac-1.

$\mathrm{R}=\mathrm{F}, \mathrm{R}^{1}=\mathrm{H}$ : rac-2.<smiles></smiles>

dihydropyrimidinone-peptoid 
Pyrimidinone-peptoid hybrid molecules $\mathbf{3 7}$ are also identified as Hsp70 modulators that inhibit cell proliferation. Trifluoromethylated hexahydropyrimidine and tetrahydropyrimidine derivatives 38-42 represent promising new leads for the development of highly potent and selective anticancer compounds and also their in vitro cytotoxic activities were determined in colon cancer cell line.<smiles>COc1ccc([C@@H]2NC(=S)N[C@](O)(C(F)(F)F)[C@H]2C(=O)c2ccco2)cc1</smiles><smiles>CCOC(=O)C1=C(C(F)(F)F)NC(=S)N[C@H]1c1ccc2c(c1)OCO2</smiles>

41<smiles>O=C(c1cccc2ccccc12)[C@H]1[C@H](c2ccc(F)cc2)NC(=S)N[C@@]1(O)C(F)(F)F</smiles>

39<smiles>O=C(c1cccc2ccccc12)[C@H]1[C@H](c2ccc3c(c2)OCO3)NC(=S)N[C@]1(O)C(F)(F)F</smiles>

40

Other structures 43-47 also have significant activity.<smiles>[X]C1=[X]=S(=O)=[X]C(C(=O)/C=C/c2ccccc2)NC(C)=C1C(=O)Nc1ccccc1Cl</smiles>

43<smiles>[Y][Y]1=C([X])C(C)=C(C(=O)NC)C(c2cccc([N+](=O)[O-])c2)N1</smiles>

44

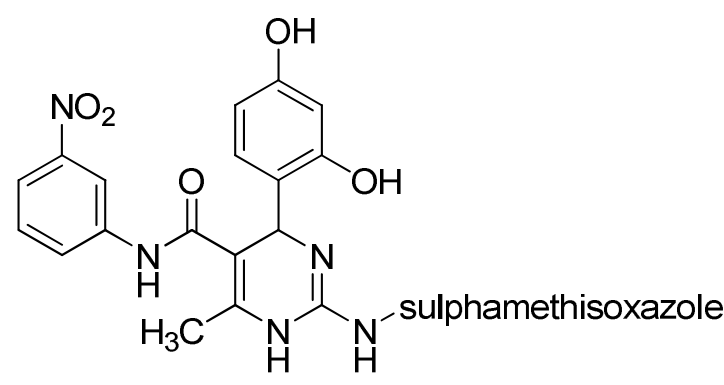

45 
<smiles>CC1=C(C(=O)Nc2cccc([N+](=O)[O-])c2)C(c2ccc(O)cc2O)N=C(S(=O)(=O)Cc2ccccc2)N1</smiles><smiles>[R]C=C1SC(=S)N(NC2=NC(c3ccc(O)cc3O)C(C(=O)Nc3cccc([N+](=O)[O-])c3)=C(C)N2)C1=O</smiles>

$\mathrm{R}=2$-furyl, 2-thienyl

47

\subsection{Anti-epileptics}

Phenobarbital 48 is well known drug for epilepsy when one sees Biginelli compounds it has similar structural framework and as a natural tendency when compounds of the type $\mathbf{4 9}$ were examined for epilepsy they have shown promising anti-epilepsy activity. ${ }^{27}$<smiles>CCC1(c2ccccc2)C(=O)NC(=O)NC1=O</smiles>

48<smiles>[R]C(=O)C1=C(C)N([R])C([X])=NC1[R]</smiles>

49

$\mathrm{R}=\mathrm{OMe}, \mathrm{Me}, \mathrm{OH}, \mathrm{NH}_{2}$, OMePh.

$\mathrm{R}^{1}=\mathrm{Ph}, 4-\mathrm{Br}-\mathrm{C}_{6} \mathrm{H}_{4}, 3-\mathrm{OMe}-\mathrm{C}_{6} \mathrm{H}_{4}$, 4- $\mathrm{NO}_{2}-\mathrm{C}_{6} \mathrm{H}_{4}, 3-\mathrm{OMe}-4-\mathrm{OH}-\mathrm{C}_{6} \mathrm{H}_{3}$, $\mathrm{C}_{3} \mathrm{H}_{7}, 3-\mathrm{OH}-\mathrm{C}_{6} \mathrm{H}_{4}$.

$\mathrm{R}^{2}=\mathrm{H}, \mathrm{Me}$.

$\mathrm{X}=\mathrm{O}, \mathrm{S}$.

\subsection{Anti-malarials}

Pyrimidinone-amides derivatives of DHPMs 50-58, a new class of Hsp70 modulators, could inhibit the replication of the pathogenic $P$. falciparum stages in human red blood cells. Nine compounds are selected as anti-malarial agents and are being investigated further. ${ }^{28}$<smiles>CCCCNC(=O)C(c1ccc(-c2ccccc2)cc1)N(CCN1CCOCC1)C(=O)CCCCN1C(=O)NC(c2ccc([N+](=O)[O-])cc2)C(C(=O)OCc2ccccc2)=C1C</smiles>

50 

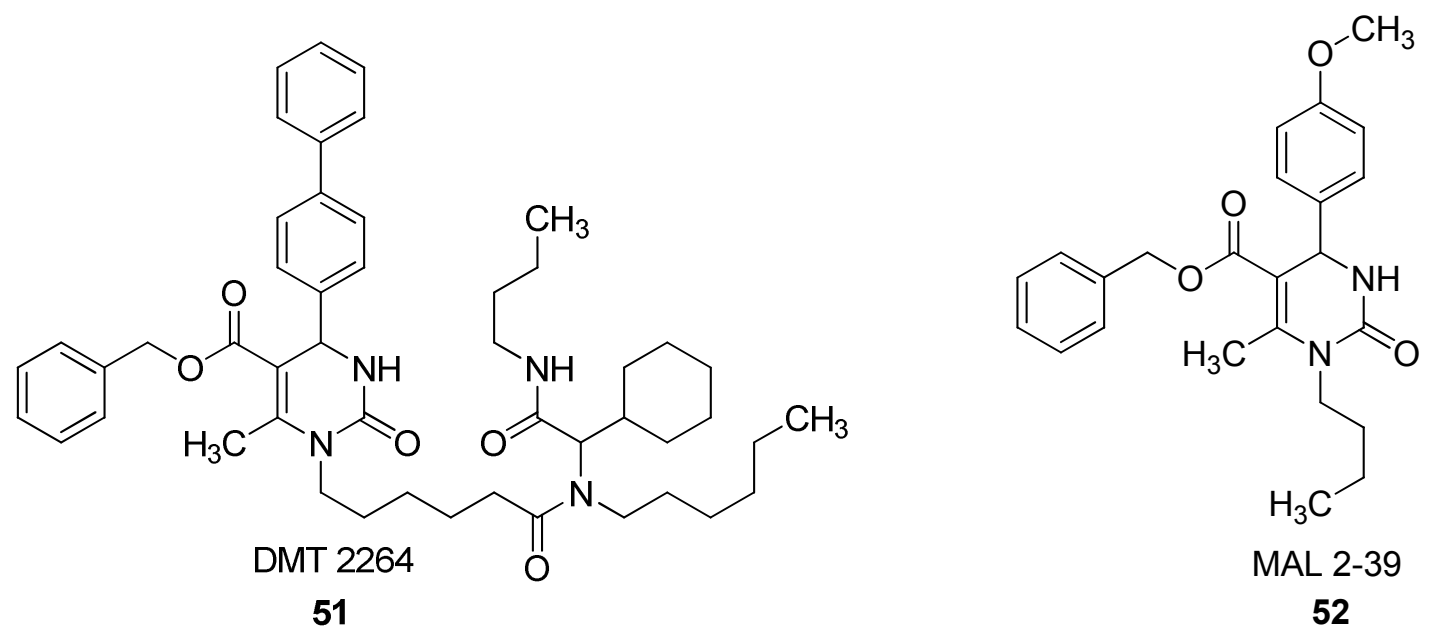

MAL 2-39

52

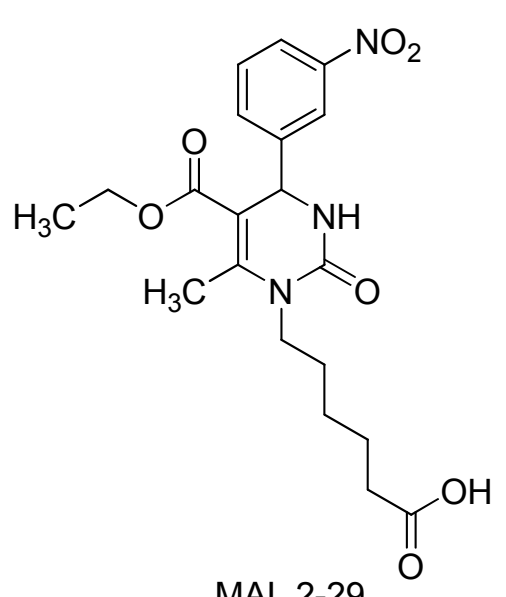

MAL 2-29

53

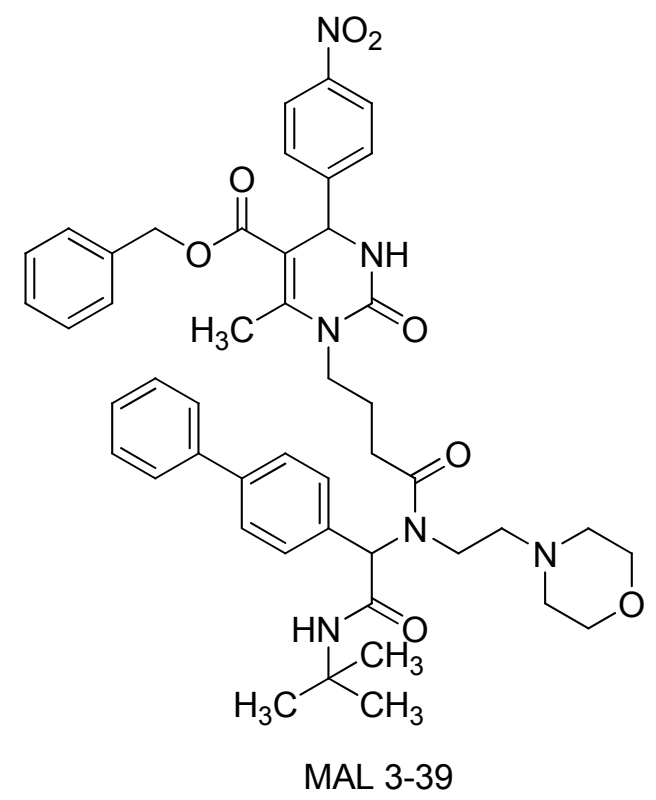

54 


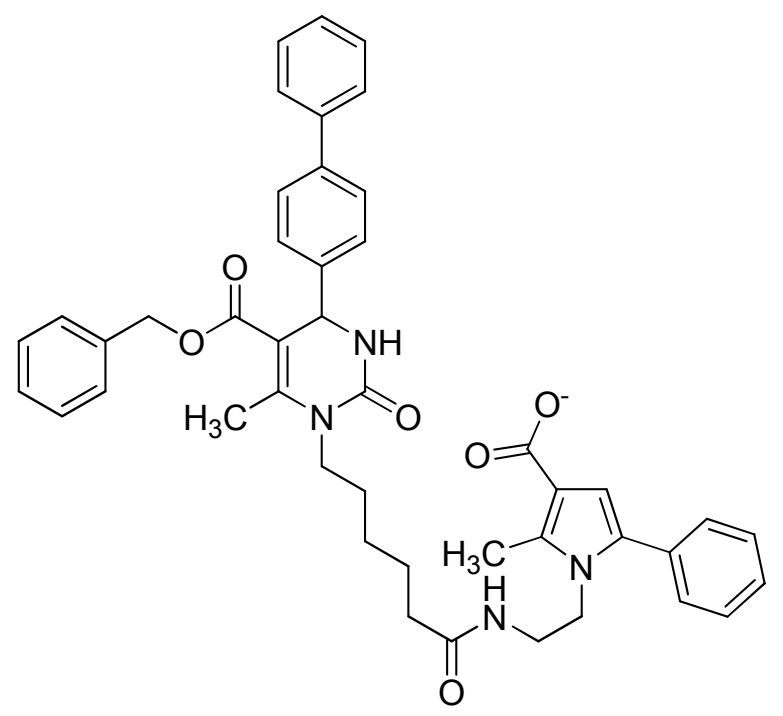

MAL 2-215

55<smiles>CCOC(=O)C1=C(C)N(CCCC(=O)NCCn2c(-c3ccccc3)cc(C(=O)[O-])c2C)C(=O)NC1c1ccc([N+](=O)[O-])cc1</smiles>

MAL 2-213

56<smiles>CCOC(=O)C1=C(C)N(Cc2ccccc2)C(=O)NC1c1ccccc1OS(=O)(=O)c1ccccc1[N+](=O)[O-]</smiles>

MAL 2-61

57<smiles>COC(=O)C1=C(Cc2ccccc2)NC(=O)NC1c1ccc2ccccc2c1</smiles>

J AB 75

58 


\subsection{Anti-microbials}

Biginelli compounds multi-functionalized with isoxazole amines i.e. 1-aryl-4-methyl-3,6-bis-(5methylisoxazol-3-yl)-2-thioxo-2,3,6,10b-tetrahydro- $1 \mathrm{H}$-pyrimido[5,4-c] quinolin-5-ones showed anti microbial also apart from antibacterial, antifungal, and antimalarial activities. ${ }^{29}$

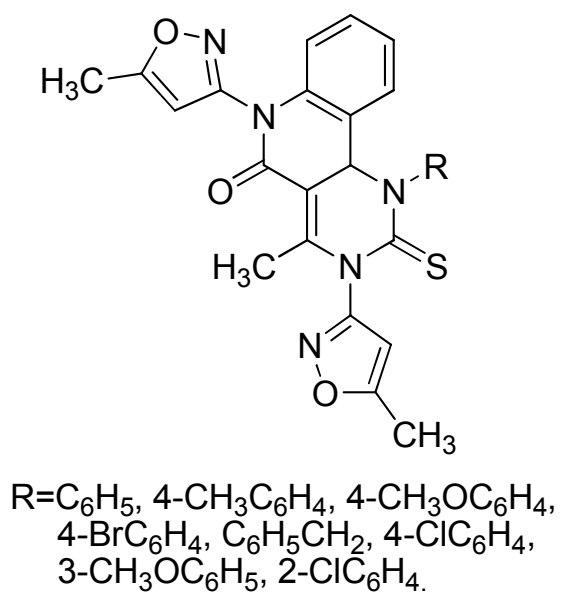

59

\subsection{Anti-inflammatories}

A series of compounds 3-(4,6-disubtituted-2-thioxo-1,2,3,4-tetrahydropyrimidin-5-yl) propanoic acid derivatives 60-62 were screened for their anti-inflammatory activity using rat paw edema method. Most of the compounds from the series showed significant anti-inflammatory activity. ${ }^{30}$<smiles>COc1cc(C2NC(=S)NC(c3ccc(C)cc3)=C2CCC(=O)O)cc(OC)c1OC</smiles><smiles>[R]c1ccc(C2=C(C=[R])C(c3ccco3)NC(=S)N2)cc1</smiles>

61<smiles>[R]OC1NC(=S)NC(c2ccccc2O)C1CCC(=O)O</smiles>

62

\subsection{Anti-tubercular activity}

Dihydropyrimidines (30 examples) also were evaluated for their antitubercular activity against Mycobacterium tuberculosis H37Rv. This study was in vitro only. Only two compounds, ethyl 4[3-(4-fluorophenyl)-1-phenyl-1H-pyrazol-4-yl]-6-methyl-2-oxo-1,2,3,4-tetrahydropyrimidine-5 carboxylate and ethyl 4-[3-(4-nitrophenyl)-1-phenyl-1H-pyrazol-4-yl]-6-methyl-2-oxo-1,2,3,4tetrahydropyrimidine-5-carboxylate $\mathbf{6 3}$ were shown to be the most active compounds and found to be more potent than isoniazid. Compounds 64 and 65 with 2,3-dimethylphenyl and 3,4- 
dimethyl carbamoyl side chain, respectively, showed $65 \%$ and $63 \%$ inhibition against Mycobacterium tuberculosis H37Rv. ${ }^{31}$<smiles>[R]OC(=O)C1=C(C)NC(=O)NC1c1cn(-c2ccccc2)nc1-c1ccc([R])cc1</smiles>

63<smiles>CC1=C(C(=O)Nc2cccc(C)c2C)C(c2cccc(Oc3ccccc3)c2)NC(=O)N1</smiles>

64<smiles>CC1=C(C(=O)Nc2ccc(C)c(C)c2)C(c2cccc([N+](=O)[O-])c2)NC(=O)N1</smiles>

65

\subsection{Anti-bacterial activity}

Ester, cyanide and some other substituted Biginelli 66-71 are reported to be promising antibacterial agents. ${ }^{32}$<smiles>CC1=C(C(=O)OC(C)C)C(c2ccccc2C)NC(=O)N1</smiles>

66<smiles>[R]c1ccc(C2NC(=O)NC(C)=C2C(=O)OC(C)C)cc1</smiles>

$\mathrm{R}=\mathrm{Cl}, \mathrm{NO}_{2}, \mathrm{~F}$.

67<smiles>[R]c1ccccc1-c1nc(N)nc(O)c1C#N</smiles>

68<smiles>[X]C(=[X])[Y]#[X]</smiles><smiles>[R]COC(=O)C1=C(C)NC2S/C(=C\c3ccccc3)C(=O)N2C1c1ccc([Si])cc1</smiles>

70<smiles>[R][R]=C([R])c1ccc(/C=C2\SC3NC(C)=C(C(=O)OCC)C(c4ccc([Si])cc4)N3C2=O)o1</smiles> 


\subsection{Miscellaneous activities}

Since our major objective in this account is to keep present description brief following structure are presented and given below them is given their activities.<smiles>[R][N+](=O)[O-]</smiles>

72<smiles>[X][Y](=O)=O</smiles>

74<smiles>CC1=C(C(=O)OC/C=C/c2ccccc2)C(c2ccccc2)NC(=O)N1</smiles>

73<smiles>CCCSC1=NC(c2ccc(F)cc2)C(C(=O)OCC)=C(C)N1</smiles>

75

Anti-oxidants $^{33} \mathbf{7 2 - 7 4}$ and Anti-filarial agents $\mathbf{7 5}$. $^{34}$<smiles>COc1cc2nc(N3CCN(C(=O)C4CCCO4)CC3)nc(C)c2cc1OC</smiles>

76<smiles>COCCCN1CCC(c2ccc(F)cc2)CC1</smiles><smiles>CNC(=O)N1C(=O)NC(COC)=C(C(=O)OC)[C@H]1c1ccc(F)c(F)c1</smiles>

78

$\alpha-1 \mathrm{~A}$ Adrenergic receptor antagonists ${ }^{35}$ 76-78. 
<smiles>COC(=O)C1=C(C)NC(c2ccccn2)=N[C@H]1c1ccc(F)cc1Cl</smiles>

79<smiles>COC(=O)C1=C(C)NC(c2ncc(F)cc2F)=N[C@H]1c1ccc(F)cc1Cl</smiles>

Bay41-4109

80<smiles>COC(=O)C1=C(C)NC(c2nccs2)=N[C@H]1c1ccc(F)cc1Cl</smiles>

Bay39-5493

81<smiles>CC1(C)CC(=O)C2=C(C1)NC(c1cccnc1)=NC2c1ccc(F)cc1Cl</smiles>

Anti-HBV (hepatitis B virus) agents ${ }^{36} \mathbf{7 9 - 8 2}$.

\section{Scope of reaction/developments in structural variants}

To develop/explore Biginelli chemistry all commonly available as well as other variants of typical reactants aldehydes, ureas and active hydrogen components have been used so far.

\subsection{Aldehyde}

In case of aldehydes all the available aliphatic, aromatic, heterocyclic and rare aldehydes have been used including sugars aldoses. Biginelli reactions of formyl- and 1,10-diformylferrocene is also reported (see Table 1).

Table 1. Aldehydes Building Blocks Used in Biginelli Reaction

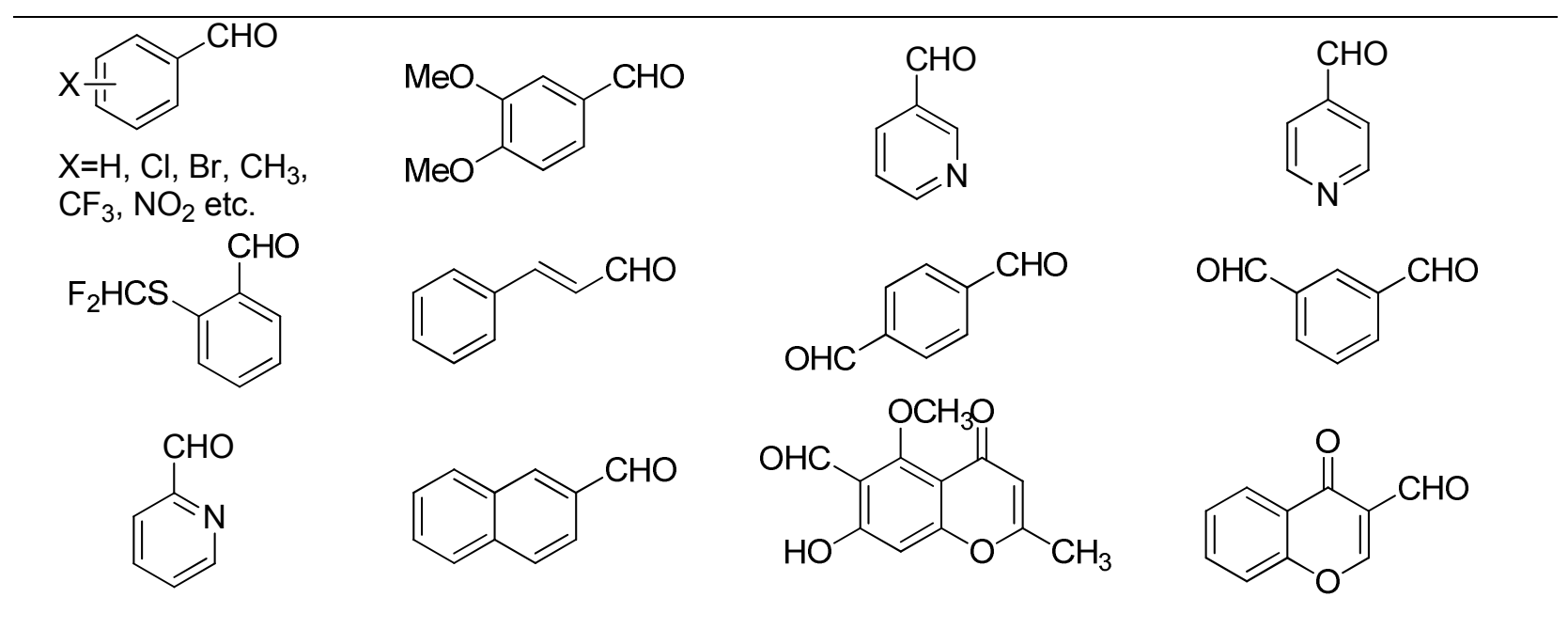




\subsection{Urea}

Regarding urea component, thiourea, and resin bonded urea and other related systems like guanidine are very successfully used. Various N-mono/di substituted ureas have been employed in this reaction to obtained pharmacologically potent molecules see Table 2 .

Table 2. Urea and thiourea building blocks used in Biginelli reactions

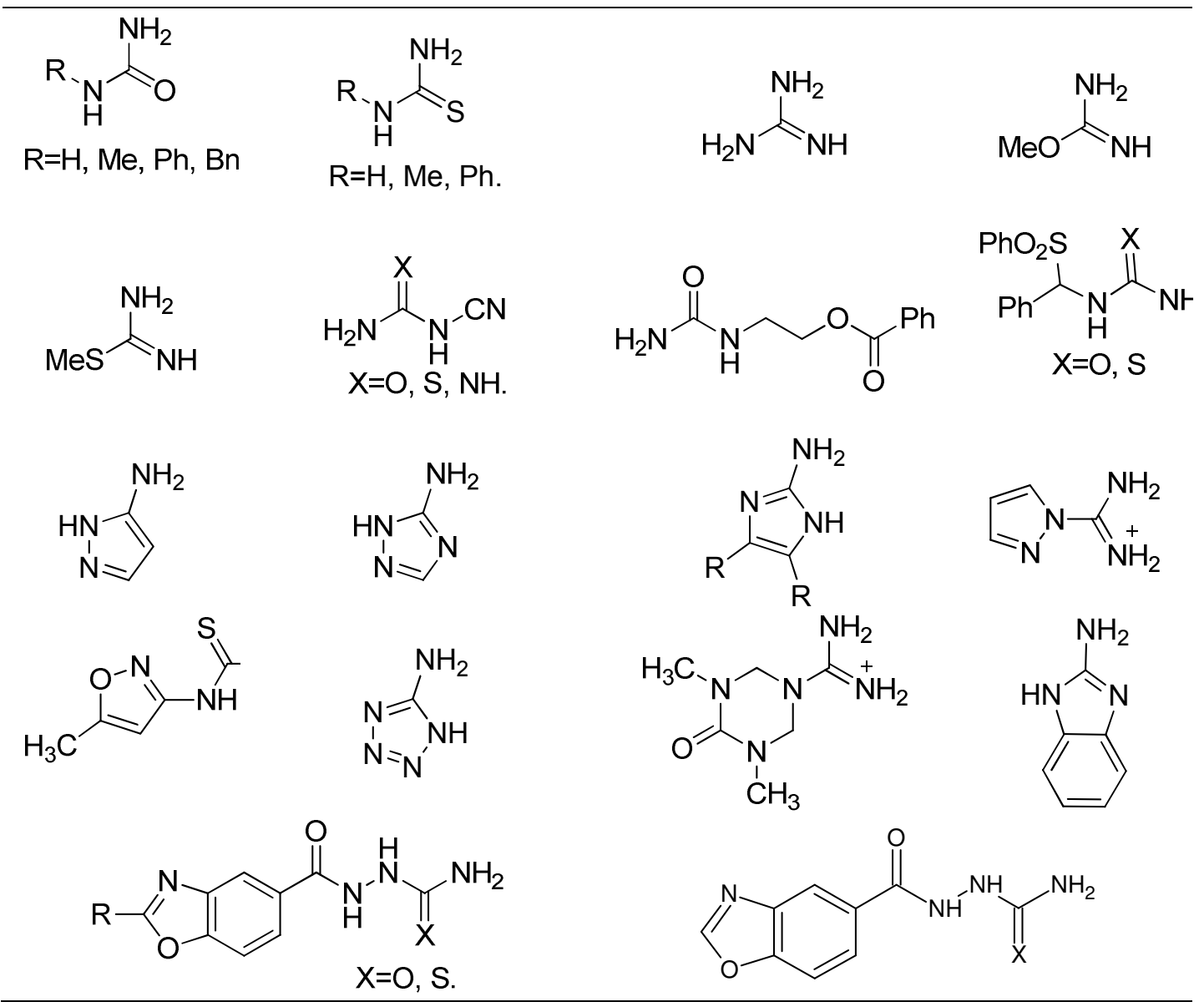

\subsection{Active hydrogen component}

In active hydrogen family varies conventional and unconventional (which can be activated) active methylene compounds have been utilized in this reaction: see Table 3. Even then there are major gaps regarding this structural partner which will be discussed in an appropriate section. Now some cases are presented below for clarity and understanding of the readership. 
Table 3. Active hydrogen building blocks used in Biginelli reactions<smiles>[R]CC(=O)c1ccc(C(=O)C=C[Al])cc1</smiles>

\subsection{Use of alcohols in place of aldehydes}

Recently, alternative to the classical synthesis of Biginelli compounds has been reported directly from aromatic alcohols under mild conditions are also reported using ionic liquid 1methylimidazolium hydrogen sulfate $[\mathrm{Hmim}] \mathrm{HSO}_{4}$. In this method aromatic aldehydes formed in situ via oxidation of aromatic alcohols with $\mathrm{NaNO}_{3}$ (Scheme 11). ${ }^{37}$ 


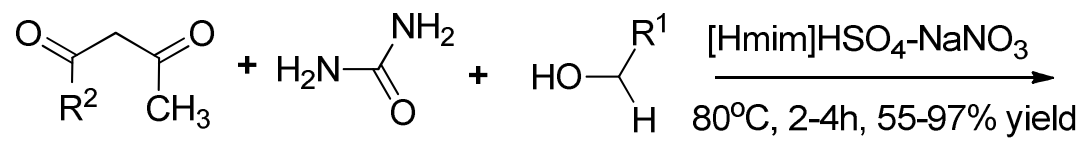<smiles>[R]C(=O)C1=C(C)NC(=O)NC1[R]</smiles>

Scheme 11. Using alcohols instead of aldehydes; synthesis of 3,4-dihydropyrimidinones.

Classically the above mentioned three components are involved in this reaction and fourth is catalyst. Medium employed in this reaction in the original report is alcohol at reflux temperature. As a natural curiosity variations have been investigated to have access to these structurally diverse Biginelli compounds. All these variables are presented in the following pages.

\section{Catalyst Variations}

Because the "privileged" nature of this scaffold is of prime importance, academic institutions were busy keeping this property as a driving force in the generation of a large number of reports in pursuit of efficient processes and procedures for these molecules. Essentially, these reports reported catalyst variations only on the following types of catalysts so far. Since several workers reported catalyst variations for the effective production of this motif authors are presenting this aspect from the latest to the earlier ones over the past decade or so: see Table 4.

Table 4. Variety of catalysts used

\section{1}

$\mathrm{I}_{2} / \mathrm{MWI},{ }^{38}$ sulfonated $\beta$-cyclodextrine, ${ }^{39} \mathrm{Cu}(\mathrm{OTf})_{2} / \mathrm{MWI},{ }^{40}$ Sulfated tungstate, ${ }^{41}$ Melamine trisulfonic acid, ${ }^{42} \mathrm{Yb}(\mathrm{PFO})_{3},{ }^{43} \mathrm{HCl} / \mathrm{EtOH},{ }^{44} \mathrm{~N}, \mathrm{~N}^{\prime}$-Dichlorobis(2,4,6-trichlorophenyl)urea (CC$2),{ }^{45} \mathrm{H}_{3} \mathrm{PO}_{3} / \mathrm{Pd}-C a t .,{ }^{46} h v / \mathrm{DMSO},{ }^{47} \mathrm{TFA} / \mathrm{THF} / \mathrm{MWI},{ }^{48} \mathrm{TMSCl} / \mathrm{CAN},{ }^{49} \mathrm{HCl}$ or $\mathrm{H}_{2} \mathrm{SO}_{4}$ or TEBA, ${ }^{50} \mathrm{FeCl}_{3} / \mathrm{Al}-\mathrm{MCM},{ }^{51}$ silica immobilized $\mathrm{Ni}(\mathrm{II}),{ }^{52} \mathrm{Ca}(\mathrm{OCl})_{2},{ }^{53} \mathrm{BPAT}-\mathrm{TfOH},{ }^{54} L-(+)-$ tartaric acid-urea mixtures, ${ }^{55} \mathrm{AcOH},{ }^{56} \mathrm{Mg}\left(\mathrm{NO}_{3}\right)_{2},{ }^{57}$ chloroacetic acid, ${ }^{58} \mathrm{PPA}-\mathrm{SiO}_{2},{ }^{59}$ gypsum, ${ }^{60}$ Nano- $\mathrm{BF}_{3} \cdot \mathrm{SiO}_{2},{ }^{61}$ Organocatalytic, ${ }^{62}$

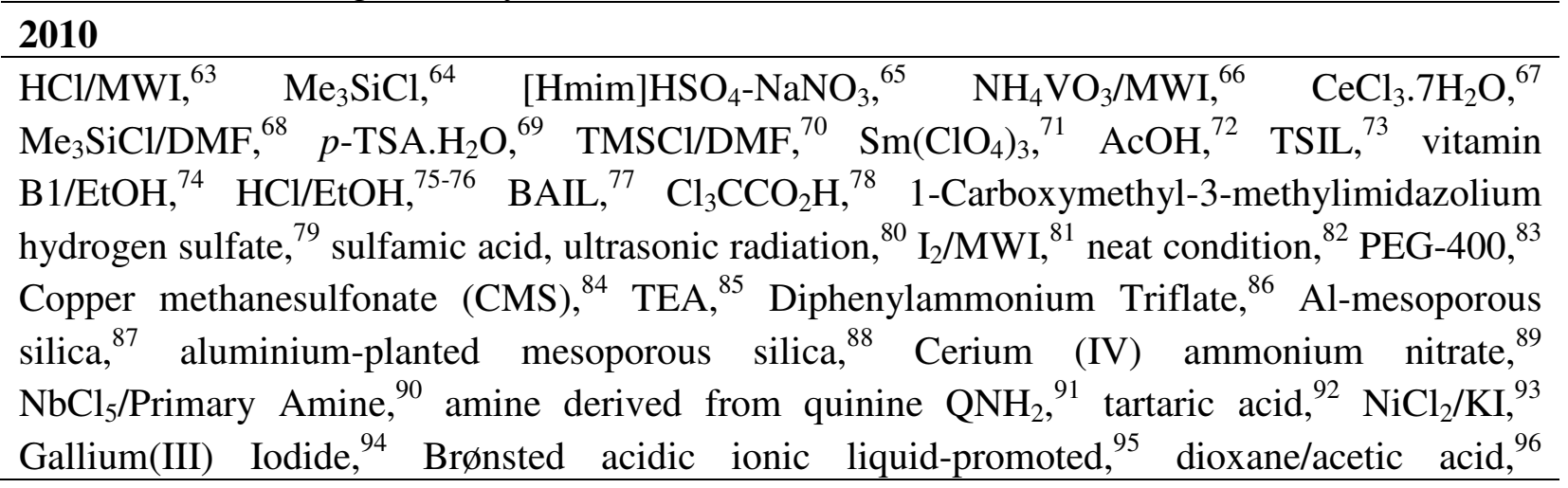


$\mathrm{NaHSO}_{4}{ }^{97}$ (diacetoxyiodo)-benzene, ${ }^{98} \quad \mathrm{TMSCl}^{99}{ }^{99} \mathrm{TiO}_{2},{ }^{100} \quad \mathrm{HBF}_{4}-\mathrm{SiO}_{2},{ }^{101} \mathrm{HCl} / \mathrm{EtOH},{ }^{102}$ $\mathrm{MgSO}_{4} \cdot 7 \mathrm{H}_{2} \mathrm{O},{ }^{103}\left[\mathrm{Bmim} \mathrm{HSO}_{4},{ }^{104}\right.$ 1,3-Dichloro-5,5-dimethylhydantoin, ${ }^{105} \mathrm{Y}(\mathrm{OAc})_{3},{ }^{106}$ Nickel Nanoparticles, ${ }^{107}$ Tributyl Borate, ${ }^{108} \mathrm{~V}\left(\mathrm{HSO}_{4}\right)_{3},{ }^{109} \mathrm{Nafion}-\mathrm{H},{ }^{110} \mathrm{CuCl}_{2},{ }^{111}$ Dodecylphosphonic Acid, ${ }^{112} p$-aminobenzene sulfonic acid, ${ }^{113}$ Phosphoric Acids, ${ }^{114}$ ammonium carbonate, ${ }^{115}$ PEGembedded thiourea dioxide, ${ }^{116}$ thiamine hydrochloride, ${ }^{117}$ hexaaquaaluminium(III) tetrafluoroborate, ${ }^{20}$ piperidinium triflate, ${ }^{118}$ aluminium-planted mesoporous silica, ${ }^{119}$ Borax, ${ }^{120}$ $\mathrm{FeCl}_{3} \cdot 6 \mathrm{H}_{2} \mathrm{O},{ }^{121} \mathrm{Cu}\left(\mathrm{NO}_{3}\right)_{2} \cdot 3 \mathrm{H}_{2} \mathrm{O},{ }^{122} \mathrm{Ce}\left(\mathrm{SO}_{4}\right)_{2}-\mathrm{SiO}_{2},{ }^{123} \mathrm{Fe}\left(\mathrm{NO}_{3}\right)_{3} \cdot 9 \mathrm{H}_{2} \mathrm{O},{ }^{124}$ aluminium-planted mesoporous silica, ${ }^{125} \mathrm{H}_{3} \mathrm{PMo}_{12} \mathrm{O}_{40},{ }^{126}$ Silica-supported tin chloride and titanium tetrachloride, ${ }^{127}$ Lewis acid, ${ }^{128}$

\section{9}

PS-PEG-SO $\mathrm{SH}_{3}{ }^{129}$ trifluoro acetic acid, ${ }^{130}$ trifluoromethane sulfonic acid, ${ }^{131}$ PS-AFDPAT, ${ }^{132}$ Yttria-Zirconia-Based Lewis Acid, ${ }^{133} \mathrm{TMSCl}^{134}{ }^{13 \mathrm{TSCl}}$ and $\mathrm{Co}(\mathrm{OAc})_{2} \cdot 4 \mathrm{H}_{2} \mathrm{O},{ }^{135}$ ytterbium chloride, ${ }^{136}$ Calcium fluoride, ${ }^{137}$ mesoporous aluminosilicate, ${ }^{138} \mathrm{Al}_{2} \mathrm{O}_{3} / \mathrm{MeSO}_{3} \mathrm{H},{ }^{139}$ Acidic Ionic Liquids, ${ }^{140} \mathrm{Al}\left(\mathrm{H}_{2} \mathrm{PO}_{4}\right)_{3},{ }^{141}$ Alumina Sulfuric Acid, ${ }^{142}$ Zinc Oxide, ${ }^{143}$ Copper Nitrate, ${ }^{144}$ Cellulose Sulfuric Acid, ${ }^{145}$ Lactic acid, ${ }^{146}$ ionic liquid, ${ }^{147} \mathrm{NaHSO}_{4} \cdot \mathrm{H}_{2} \mathrm{O},{ }^{148} \mathrm{H}_{2} \mathrm{SO}_{4}$ Supported on Silica Gel or Alumina, ${ }^{149} \mathrm{Al}\left(\mathrm{HSO}_{4}\right)_{3}$ and $\mathrm{Al}_{2} \mathrm{O}_{3}-\mathrm{SO}_{3} \mathrm{H},{ }^{150} \mathrm{NaH},{ }^{151} \mathrm{SnCl}_{2},{ }^{152} \mathrm{TBAB},{ }^{153}$ $\mathrm{Mg} / \mathrm{MeOH},{ }^{154} \mathrm{PTSA}$,

$\mathrm{Sc}(\mathrm{OTf})_{3}$ or La(OTf) $)_{3},{ }^{155}$ L-proline/TFA, ${ }^{156} \mathrm{NaCl}^{6(1)}$ Copper(II) Sulfamate, ${ }^{157} \mathrm{I}_{2} / \mathrm{MWI},{ }^{158} \mathrm{UV}$ irradiation, ${ }^{159} \mathrm{I}_{2},{ }^{160}$ Amberlyst-70, ${ }^{161}$ Etidronic Acid, ${ }^{162} \mathrm{Fe} 3+, \mathrm{Co}+, \mathrm{Zn} 2+$, Li+ salts, ${ }^{163}$ NBS/silica sulfuric acid/MWI, ${ }^{164}$ 1,3-Dibromo-5,5-dimethylhydantoin $(\mathrm{DBH}){ }^{165}$ Uncatalysed, ${ }^{166}$ oxone on wet alumina or hydrogen peroxide-vanadyl sulfate, ${ }^{167} \mathrm{PdO},{ }^{168}$ Indion $130,{ }^{169} \mathrm{TMSCl} / \mathrm{DMF},{ }^{170}$ Proline Ester Salts, ${ }^{171} \mathrm{HCO}_{2} \mathrm{H},{ }^{18}$ CBPA-Thiurea, ${ }^{172} \mathrm{Yb}-$ bis(perfluorooctanesulfonyl)imide, ${ }^{173} \mathrm{I}_{2},{ }^{174}$ phosphinite ionic liquid, ${ }^{175}$ Organocatalyst, ${ }^{176}$ alumina-supported trifluoromethane sulfonic acid, ${ }^{177}$ etidronic acid, ${ }^{178}$ zinc tetrafluoroborate, ${ }^{179}$ ammonium dihydrogen orthophosphate, ${ }^{180}$ lipase, ${ }^{181} \mathrm{Na}_{2} \mathrm{SeO}_{4},{ }^{182}$ Bifunctional Organocatalyst, ${ }^{183} \mathrm{NiSO}_{4} .7 \mathrm{H}_{2} \mathrm{O},{ }^{184} \mathrm{NaIO}_{4},{ }^{185}$ Phosphoric Acids. ${ }^{186}$

\section{8}

$\mathrm{MgCl}_{2} / \mathrm{AcOH},{ }^{187} \mathrm{MCM}-41-\mathrm{R}-\mathrm{SO}_{3} \mathrm{H}^{188}{ }^{18} \mathrm{MWI},{ }^{189} \mathrm{ZrCl}_{4} /$ Ultrasound, ${ }^{190}$ Titanium(IV) Chloride, ${ }^{191}$ $\mathrm{HCl} / \mathrm{AcOH} / \mathrm{MWI},{ }^{192} \mathrm{KF}-$ alumina, ${ }^{193} \mathrm{HCl}, \mathrm{p}-\mathrm{TsOH}$ or $\mathrm{H}_{2} \mathrm{NSO}_{3} \mathrm{H},{ }^{194} \mathrm{PhI}(\mathrm{OAc})_{2} / \mathrm{t}-\mathrm{BuOOH},{ }^{195}$ PPE ${ }^{196}$ L-prolinium sulfate, ${ }^{197}\left[\mathrm{HOC}_{2} \mathrm{mim}\right][\mathrm{PF} 6] / \mathrm{MWI},{ }^{198} \mathrm{HCl} / \mathrm{AcONa},{ }^{199} \mathrm{LaCl}_{3} / \mathrm{ethanol},{ }^{200}$ Ultrasonification, ${ }^{201} \mathrm{H}_{2} \mathrm{NSO}_{3} \mathrm{H}, \mathrm{TMSCl}^{202} \mathrm{Me}_{3} \mathrm{SiCl}^{203}$ Zeolite, ${ }^{204} \mathrm{Cu}(\mathrm{TFA})_{2} \cdot 4 \mathrm{H}_{2} \mathrm{O},{ }^{205}$ $\mathrm{LaCl}_{3}$.Hydrates, ${ }^{206} \mathrm{Pr}\left(\mathrm{CH}_{3} \mathrm{SO}_{3}\right)_{3} .2 \mathrm{H}_{2} \mathrm{O},{ }^{207}$ 2-chloro-4-nitrobenzoic acid/trans-4-hydroxyproline derivative, ${ }^{208}$ Ferric Perchlorate, ${ }^{209}$ Polystyrene-supported $\mathrm{AICl}_{3},{ }^{210} \mathrm{DDQ},{ }^{211}$ Oxalic acid, ${ }^{212}$ $\mathrm{AcOH},{ }^{213}$ tungstate sulfuric acid, ${ }^{214} \mathrm{KHSO}_{4}$ and $\mathrm{HCl},{ }^{215} \mathrm{MWI},{ }^{216} \mathrm{SnCl}_{4},{ }^{217} \mathrm{CuCl}_{2} .2 \mathrm{H}_{2} \mathrm{O}-\mathrm{HCl},{ }^{218}$ citric acid, ${ }^{219} \mathrm{~Pb}(\mathrm{NO}),{ }^{220} \mathrm{HCl}^{221}{ }^{2 i B r},{ }^{222}\left[\mathrm{Bmim}^{2}\left[\mathrm{FeCl}_{4}\right],{ }^{223} \mathrm{TMSCl} / \mathrm{DMF},{ }^{224}\right.$ Lanthanide halides derived from mischmetal $\left(\mathrm{LnCl}_{3} \times 7 \mathrm{H}_{2} \mathrm{O}\right) / \mathrm{HCl} / \mathrm{EtOH},{ }^{225} \mathrm{LaCl}_{3 / \text { graphite/HCl} / \mathrm{MWI}}{ }^{226}$ Aluminia-sulfuric acid, ${ }^{227} \mathrm{Fe}\left(\mathrm{HSO}_{4}\right)_{3},{ }^{228} \mathrm{CuCl}_{2} \times 2 \mathrm{H}_{2} \mathrm{O} / \mathrm{Cl}_{2} \mathrm{H}_{2} 5 \mathrm{SO}_{3} \mathrm{Na} / \mathrm{H}_{2} \mathrm{O},{ }^{229}$ Pyrazolidine dihydrochloride, ${ }^{230} \mathrm{TiCl}_{4},{ }^{231} \mathrm{SiCl}_{4} / \mathrm{DMF},{ }^{232}$ Sulfonic salicylic acid, ${ }^{233} \operatorname{Pr}\left(\mathrm{MeSO}_{3}\right)_{3},{ }^{234} \mathrm{CuCl}_{2} \times$ $2 \mathrm{H}_{2} \mathrm{O} / \mathrm{HCl} /$ grinding, ${ }^{235} \mathrm{Ph}_{3} \mathrm{P},{ }^{236} \quad \mathrm{H}_{3} \mathrm{PW}_{12} \mathrm{O}_{40}{ }^{237} \quad$ Scolecite, ${ }^{238} \quad \mathrm{HClO}_{4}-\mathrm{SiO}_{2},{ }^{239} \quad \mathrm{ZrO}_{2-}$ nanopowder/MWI, ${ }^{240} \mathrm{~Pb}\left(\mathrm{NO}_{3}\right)_{2} .{ }^{241}$ 


\section{7}

$\mathrm{Zn}\left(\mathrm{ClO}_{4}\right)_{2} \times 6 \mathrm{H}_{2} \mathrm{O},{ }^{242} \mathrm{TsOH}^{243} \mathrm{VCl}_{3}{ }^{244} \mathrm{TEBA}^{245} \mathrm{HClO}_{4} / \mathrm{MWI},{ }^{246}$ Polystyrenesulfonic acid (PSSA)/MWI, ${ }^{247} \mathrm{Bi}\left(\mathrm{NO}_{3}\right)_{3},{ }^{248}$ Bakers yeast, ${ }^{249} \mathrm{HCO}_{2} \mathrm{H} / \mathrm{MWI},{ }^{250} \mathrm{SiO}_{2}-\mathrm{Si}\left(\mathrm{CH}_{2}\right)_{3} \mathrm{SO}_{3} \mathrm{H}^{251}$ Metallophthalocyanines, ${ }^{252}$ Nafion-H resin, ${ }^{253}$ Amino Acetic Acid, ${ }^{254} \mathrm{AlCl}_{3},{ }^{255} \mathrm{PhCO}_{2} \mathrm{H}^{256}$ $\mathrm{MgSO}_{4},{ }^{257} \mathrm{SiO}_{2} / \mathrm{ZnCl}_{2},{ }^{258}$ Silica sulfuric acid, ${ }^{259} \mathrm{H}_{4} \mathrm{SiW}_{12} \mathrm{O}_{40} \mathrm{nH}_{2} \mathrm{O},{ }^{260}\left[\mathrm{C}_{4} \mathrm{mim}\right]\left[\mathrm{HSO}_{4}\right] / \mathrm{MWI}^{261}$ $\mathrm{R}_{3} \mathrm{~N}^{+} \quad\left(\mathrm{CH}_{2}\right)_{3} \mathrm{SO}_{3}{ }^{-262}, \quad[\mathrm{Hmim}] \mathrm{HSO}_{4},{ }^{263} \quad \mathrm{H}_{6} \mathrm{P}_{2} \mathrm{~W}_{18} \mathrm{O}_{62} \times 24 \mathrm{H}_{2} \mathrm{O},{ }^{264} \mathrm{ZnBr}_{2},{ }^{265} \mathrm{TiCl}_{3}{ }^{266}$ $\mathrm{ZrOCl}_{2} \times 8 \mathrm{H}_{2} \mathrm{O}$ or $\mathrm{ZrCl}_{4} /$ neat, ${ }^{267} \mathrm{Y}\left(\mathrm{NO}_{3}\right)_{3} \times 6 \mathrm{H}_{2} \mathrm{O}$, ${ }^{268} \mathrm{AlCl}_{3}$ or $\mathrm{AlBr}_{3}{ }^{269} \mathrm{GaCl}_{3} / \mathrm{MWI}$ or $\mathrm{GaBr}_{3} / \mathrm{MWI},{ }^{14}$ Ziegler-Natta catalyst system $\left(\mathrm{TiCl}_{4}-\mathrm{MgCl}_{2}-4 \mathrm{CH}_{3} \mathrm{OH}\right),{ }^{270} \mathrm{HClO}_{4},{ }^{271} \mathrm{SmI}_{2},{ }^{272}$ $\mathrm{NaBF}_{4},{ }^{273} \mathrm{DSA}^{274}$ PEG $400,{ }^{275} \mathrm{TMSCl}^{2} \mathrm{MWI},{ }^{276}$ Silica triflate, ${ }^{277}$ Silica-chloride, ${ }^{278}$ TMSCl/DMF, ${ }^{279} \mathrm{TMSCl} / \mathrm{DMF} /$ sonication, ${ }^{280} \mathrm{ClCH}_{2} \mathrm{CO}_{2} \mathrm{H},{ }^{281} \mathrm{CuI} / \mathrm{H}_{2} \mathrm{O},{ }^{282} \mathrm{HBF}_{4},{ }^{283}$

Trichloroisocyanuric acid, ${ }^{284} \mathrm{H}_{4} \mathrm{PMo}_{11} \mathrm{VO}_{40}{ }^{285} \mathrm{KH}_{2} \mathrm{PO}_{4} / \mathrm{glycol},{ }^{286} \mathrm{FeCl}_{3} \times \quad 6 \mathrm{H}_{2} \mathrm{O} / \mathrm{MWI},{ }^{287}$ Nafion-H resin, ${ }^{288} \mathrm{CuCl}_{2} \times 2 \mathrm{H}_{2} \mathrm{O} / \mathrm{MWI},{ }^{289} \mathrm{SbCl}_{3},{ }^{16} \mathrm{TaBr}_{5},{ }^{290}$ Propane phosphonic acid anhydride $\left(\mathrm{n}-\mathrm{C}_{3} \mathrm{H}_{7} \mathrm{PO}_{2}\right)_{3} / \mathrm{AcOEt},{ }^{291} \quad \mathrm{ZnI}_{2} / \mathrm{MWI},{ }^{292} \quad \mathrm{KHSO}_{4},{ }^{293} \quad \mathrm{P}_{2} \mathrm{O}_{5}{ }^{294} \quad \mathrm{HCl} / \mathrm{AcOH},{ }^{295}$ $\mathrm{Cu}\left(\mathrm{BF}_{4}\right)_{2} \times \mathrm{xH}_{2} \mathrm{O} /$ neat, ${ }^{296} \mathrm{Fe}\left(\mathrm{CF}_{3} \mathrm{CO}_{2}\right)_{3}$ or $\mathrm{Fe}(\mathrm{OTf})_{3},{ }^{297} \mathrm{MoO}_{3} / \mathrm{Al}_{2} \mathrm{O}_{3},{ }^{298} \mathrm{Ca}\left(\mathrm{HSO}_{4}\right)_{2}, \mathrm{Zn}\left(\mathrm{HSO}_{4}\right)_{2}$ or Oxone, ${ }^{299} \mathrm{Bi}\left(\mathrm{NO}_{3}\right)_{3},{ }^{300}[\mathrm{BMIm}] \mathrm{Sac},{ }^{301} \mathrm{Cu} /$ silica xerogel composite, ${ }^{302}$ Silica sulfuric $\mathrm{acid} /[\mathrm{Bmim}] \mathrm{Br},{ }^{303}$ BF3.Et $2 \mathrm{O} / \mathrm{PhMe} / \mathrm{Mol}$. Sieves, ${ }^{304} \mathrm{~K}-10$ clay/MWI, ${ }^{305}\left[\mathrm{HOC}_{2} \mathrm{mim}\right]\left[\mathrm{PF}_{6}\right],{ }^{306}$ $\mathrm{Co}\left(\mathrm{NO}_{3}\right)_{2} \cdot 6 \mathrm{H}_{2} \mathrm{O} / \mathrm{K}_{2} \mathrm{~S}_{2} \mathrm{O}_{8},{ }^{307}[\mathrm{bmim}] \mathrm{BF}_{4}$ immobilized $\mathrm{Cu}(\mathrm{acac})_{2}{ }^{308}$

\section{6}

$\mathrm{NaHCO}_{3} / \mathrm{DMF},{ }^{309} p$-TSA/MWI, ${ }^{310} n$-BuLi/THF, ${ }^{311} \mathrm{CAN} / \mathrm{NaHCO}_{3},{ }^{312} \mathrm{PEG} \mathrm{SO}_{3} \mathrm{H}^{313}{ }^{31} \mathrm{SbCl}_{3}$ on alumina, $^{314}$ Sulfated zirconia, ${ }^{315}$ Sulfated zirconia, ${ }^{316}$ EtOH/AcOH, ${ }^{317}$ EtOH/AcOH, ${ }^{318}$ $\mathrm{NH}_{2} \mathrm{SO}_{3} \mathrm{H} /$ grinding, $\left.{ }^{319} \mathrm{BMim}\right]\left[\mathrm{BF}_{4}\right],{ }^{320} \quad\left[\mathrm{CMIm}_{[}\left[\mathrm{HSO}_{4}\right],{ }^{321}\right.$ 1-n-Butyl-3-methylimidazolium saccharinate [BMIm]Sac), ${ }^{322}$ Dowex-50W, ${ }^{323} \quad \mathrm{~K}_{5} \mathrm{CoW}_{12} \mathrm{O}_{40} 3 \mathrm{H}_{2} \mathrm{O},{ }^{324} \quad \mathrm{H}_{3} \mathrm{PW}_{12} \mathrm{O}_{40} /$ sulfated zirconia/MWI, ${ }^{325} \mathrm{TMSCl} / \mathrm{DMF},{ }^{326}\left(\mathrm{NH}_{4}\right)_{2} \mathrm{HPO}_{4},{ }^{327} \mathrm{Ce}\left(\mathrm{NO}_{3}\right)_{3} 6 \mathrm{H}_{2} \mathrm{O},{ }^{328}$ Binol- and $\mathrm{H}_{8}$-binolbased phosphoric acids, ${ }^{329}$ Polyaniline salts \& complexes, ${ }^{330} \mathrm{ZrOCl}_{2} \times 8 \mathrm{H}_{2} \mathrm{O},{ }^{331}$ Zeolite, ${ }^{332}$ (L)Pro-OMe $\times \mathrm{HCl}^{333} \mathrm{Cu}\left(\mathrm{NTf}_{2}\right)_{2}$, or $\mathrm{Ni}\left(\mathrm{NTf}_{2}\right)_{2}$, or $\mathrm{Yb}\left(\mathrm{NTf}_{2}\right)_{3},{ }^{334} \mathrm{PhB}(\mathrm{OH})_{2},{ }^{335} \mathrm{Zn}\left(\mathrm{NH}_{2} \mathrm{SO}_{3}\right)_{2},{ }^{336}$ $\mathrm{Zn}\left(\mathrm{NH}_{2} \mathrm{SO}_{3}\right)_{2},{ }^{337}$ Dowex-50W, ${ }^{338} \mathrm{RuCl}_{3} \times \mathrm{nH}_{2} \mathrm{O},{ }^{339} \mathrm{H}_{2} \mathrm{SO}_{4},{ }^{340} \mathrm{CuBr}_{2},{ }^{341} \mathrm{PEG}-\mathrm{SO}_{3} \mathrm{H} / \mathrm{MWI},{ }^{342}$ $\mathrm{NH}_{4} \mathrm{Cl} /$ ultrasound irradiation, ${ }^{33(\mathrm{a})} \mathrm{Sr}\left(\mathrm{NO}_{3}\right)_{2},{ }^{343} \mathrm{H}_{3} \mathrm{PMo}_{12} \mathrm{O}_{4},{ }^{344}$ Supported $\mathrm{H}_{3} \mathrm{PW}_{12} \mathrm{O}_{40}$ or $\mathrm{H}_{3} \mathrm{PMo}_{12} \mathrm{O}_{40},{ }^{345}$ Keggin-type heteropolyacids- $\mathrm{H}_{3} \mathrm{PW}_{12} \mathrm{O}_{40}, \mathrm{H}_{3} \mathrm{PMo}_{12} \mathrm{O}_{40}$ or $\mathrm{H}_{4} \mathrm{SiW}_{12} \mathrm{O}_{40}{ }^{346}$ $\mathrm{H}_{3} \mathrm{PW}_{12} \mathrm{O}_{40}$ or $\mathrm{H}_{3} \mathrm{PW}_{12} \mathrm{O}_{40}-\mathrm{SiO}_{2},{ }^{347}$ Ion exchange resin Nafion NR-50, ${ }^{348} \mathrm{H}_{3} \mathrm{PW}_{12} \mathrm{O}_{40} / \mathrm{SiO}_{2},{ }^{349}$ $\mathrm{KAl}\left(\mathrm{SO}_{4}\right)_{2} \times 12 \mathrm{H}_{2} \mathrm{O} / \mathrm{SiO}_{2},{ }^{350} \quad \mathrm{Zn}\left(\mathrm{NO}_{3}\right)_{2},{ }^{351} \quad \mathrm{ClSO}_{3} \mathrm{H}^{352}{ }^{352} \quad[\mathrm{BPy}] \mathrm{BF}_{4},{ }^{353} \mathrm{I}_{2} /$ ultrasound, ${ }^{354}$ $\mathrm{H}_{2} \mathrm{SO}_{4} / \mathrm{MWI},{ }^{355} \beta$-Cyclodextrin/ $/ \mathrm{HCl},{ }^{356}$ two-phase system, ${ }^{357} \mathrm{H}_{3} \mathrm{PW}_{12} \mathrm{O}_{40} / \mathrm{MWI},{ }^{358} \mathrm{Mg}\left(\mathrm{ClO}_{4}\right)_{2} /$ ultrasound. ${ }^{359}$

\section{5}

$\mathrm{Bi}\left(\mathrm{NO}_{3}\right)_{3} .5 \mathrm{H}_{2} \mathrm{O},{ }^{360}$ Formylphenylboronic acid, ${ }^{361}$ IL phase-linked aldehyde, ${ }^{362} \mathrm{HCl}^{363}$ $\mathrm{SrCl}_{2} \cdot 6 \mathrm{H}_{2} \mathrm{O}-\mathrm{HCl},{ }^{364} \mathrm{H}_{3} \mathrm{BO}_{3},{ }^{365} \mathrm{TMSCl}^{366} \mathrm{SmI}_{2},{ }^{367} \mathrm{FeCl}_{3} \cdot 6 \mathrm{H}_{2} \mathrm{O},{ }^{368}$ Ionic liquids (IL), ${ }^{369}$ Polymer-supported IL, ${ }^{370} \mathrm{Me}_{3} \mathrm{SiI}^{371} \mathrm{Yb}(\mathrm{OTf})_{3} /$ chiral hexadentate amino phenol ligand, ${ }^{372}$ Sulphated $\mathrm{SnO}_{2}{ }^{373} \mathrm{ZnCl}_{2} / \mathrm{MWI},{ }^{374} \mathrm{BiOClO}_{4} \cdot \mathrm{nH}_{2} \mathrm{O},{ }^{375} \mathrm{CoCl}_{2} \cdot 6 \mathrm{H}_{2} \mathrm{O} / \mathrm{MWI}, \mathrm{MnCl}_{2} .4 \mathrm{H}_{2} \mathrm{O} / \mathrm{MWI}$, $\mathrm{SnCl}_{2} \cdot 2 \mathrm{H}_{2} \mathrm{O} / \mathrm{MWI},{ }^{376} \mathrm{Bi}\left(\mathrm{NO}_{3}\right)_{3},{ }^{377} \quad \mathrm{TEBA}^{378}$ Polyaniline-fluoroboric aciddodecylhydrogensulfate salt $\left(\mathrm{PANIHBF}_{4}-\mathrm{DHS}\right),{ }^{379} \operatorname{In}(\mathrm{OTf})_{3},{ }^{380} \mathrm{RuCl}_{3},{ }^{381} \mathrm{MeSO}_{3} \mathrm{H}^{382}$ $\mathrm{Sc}(\mathrm{OTf})_{3}{ }^{383} \mathrm{MgCl}_{2} \times 6 \mathrm{H}_{2} \mathrm{O},{ }^{384}$ Natural phosphate doped with metal halides $\mathrm{MCl}_{2}$ 
$(\mathrm{M}=\mathrm{Cu}, \mathrm{Zn}, \mathrm{Co}, \mathrm{Ni}) / \mathrm{PhMe}^{385} \quad \mathrm{CuCl}_{2} / \mathrm{LiCl},{ }^{386} \quad$ Uronium hydrogen sulfates, ${ }^{387} \mathrm{TiCl}_{4},{ }^{388}$ $\mathrm{CaAl}_{2} \mathrm{Si}_{7} \mathrm{O}_{18} \times 6 \mathrm{H}_{2} \mathrm{O},{ }^{389} \mathrm{Si}(\mathrm{OEt})_{4} / \mathrm{FeCl}_{3},{ }^{390} \mathrm{Sr}(\mathrm{OTf})_{2},{ }^{391} \mathrm{H}_{3} \mathrm{PW}_{12} \mathrm{O}_{40},{ }^{392} \mathrm{I}_{2} / \mathrm{Al}_{2} \mathrm{O}_{3} / \mathrm{MWI},{ }^{393} \mathrm{CeO}_{2}$ supported on poly(4-vinylpyridine-co-divinylbenzene)(PVP-DVB)/ $\mathrm{H}_{2} \mathrm{O},{ }^{394}$ Fluoroapatite $\mathrm{Ca}_{10}\left(\mathrm{PO}_{4}\right)_{6} \mathrm{~F}_{2} / \mathrm{MCl}_{2} /$ toluene $(\mathrm{M}=\mathrm{Zn}, \mathrm{Cu}, \mathrm{Ni}),{ }^{395} n-\mathrm{Bu}_{2} \mathrm{SnO}^{396}$ [BMIm]Sac, ${ }^{397}$ Expansive graphite, ${ }^{398}$ Hydroxyapatite doped

with $\mathrm{ZnCl}_{2}, \mathrm{CuCl}_{2}, \mathrm{NiCl}_{2}$ and $\mathrm{CoCl}_{2},{ }^{399} \mathrm{NH}_{2} \mathrm{SO}_{3} \mathrm{H}^{400} h v / \mathrm{MeOH},{ }^{401}$ 1-[2-(4formylbenzoyloxy) ethyl]pyridiniumhexafluorophosphate. ${ }^{402}$

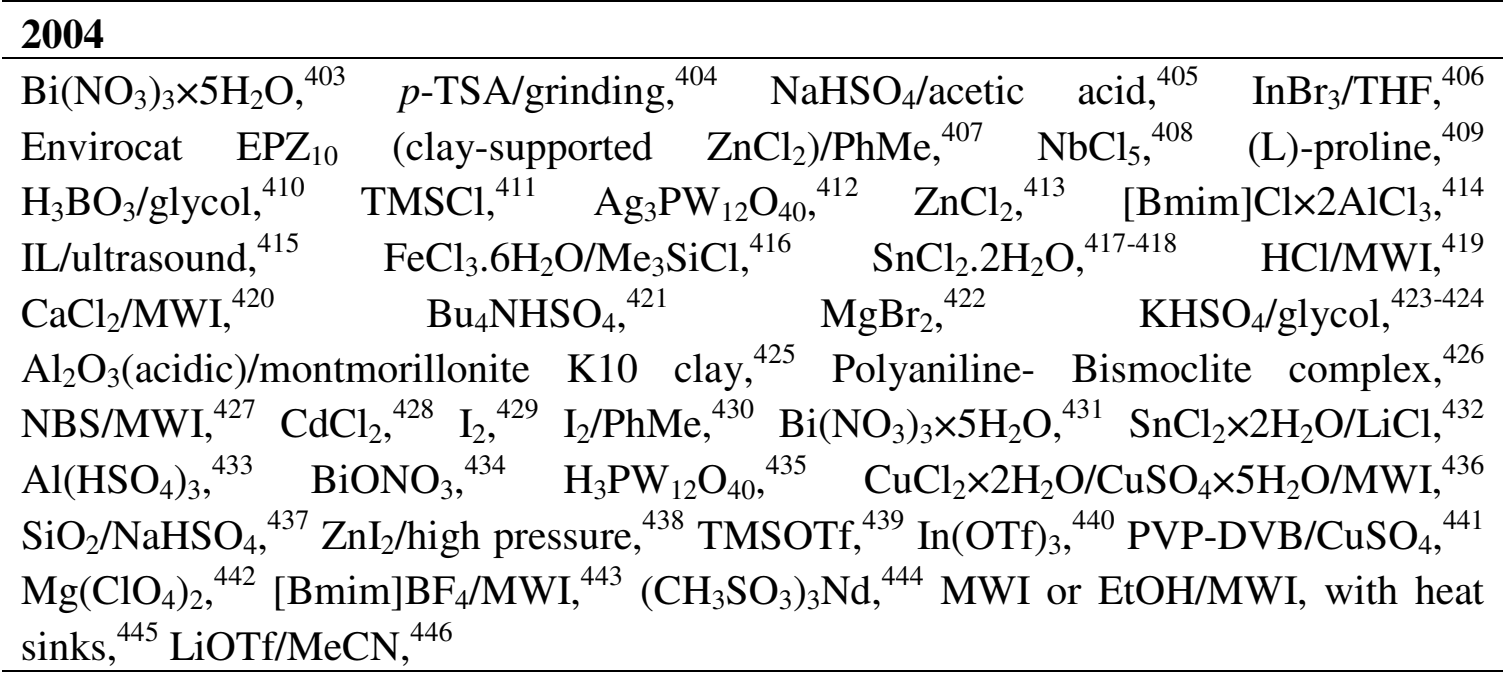

2003

Multistep polymer assisted synthesis, ${ }^{447} \quad \mathrm{VCl}_{3},{ }^{448}$ TAFF/neat/IR $\quad$ light, ${ }^{449}$ AcOH/MWI ${ }^{450}{ }^{\mathrm{LiBr}},{ }^{451} \mathrm{NH}_{2} \mathrm{SO}_{3} \mathrm{H} / \mathrm{ultrasound},{ }^{452}$ Silica sulfuric acid, ${ }^{453}$ Silica sulfuric acid ${ }^{454} \mathrm{Bi}(\mathrm{OTf})_{3},{ }^{455}$ Montmorillonite $\mathrm{KSF}$ clay/MWI, ${ }^{456}$ TMSI (TMSCl/NaI), ${ }^{457}$ PPA/PEG400 support/MWI ${ }^{458} \mathrm{Yb}(\mathrm{OTf})_{3},{ }^{459} \mathrm{Yb}(\mathrm{OTf})_{3}$ or $\mathrm{Sc}(\mathrm{OTf})_{3}$ or $\mathrm{La}(\mathrm{OTf})_{3},{ }^{460}$ $\mathrm{Yb}(\mathrm{OTf})_{3} / 4 \AA^{\prime}$ mol. sieves, ${ }^{461} \mathrm{CeCl}_{3}, \mathrm{InCl}_{3},{ }^{462} \mathrm{In}(\mathrm{OTf})_{3} / \mathrm{MWI} / \mathrm{Na}_{2} \mathrm{SO}_{4}$ solid support, ${ }^{463}$ $\mathrm{Al}_{2} \mathrm{O}_{3}$ (acidic)/MWI, ${ }^{464} \mathrm{TsOH} / \mathrm{MWI},{ }^{465} \mathrm{Zn}(\mathrm{OTf})_{2},{ }^{466} \mathrm{SmCl}_{3} \times 6 \mathrm{H}_{2} \mathrm{O} / \mathrm{Montmorillonite}$ clay/MWI ${ }^{467}$ PPE/MWI, ${ }^{468}$ PPE/MWI ${ }^{469} \mathrm{hv},{ }^{470} \mathrm{CeCl}_{3} \times 7 \mathrm{H}_{2} \mathrm{O},{ }^{471} \mathrm{InBr}_{3}$ or $\mathrm{InCl}_{3} \times 4 \mathrm{H}_{2} \mathrm{O},{ }^{472} \mathrm{Cu}(\mathrm{OTf})_{2},{ }^{473} \quad \mathrm{PhCH}(\mathrm{Me}) \mathrm{N}^{+} \mathrm{Me}_{2} \mathrm{BuBr}-,{ }^{474} \quad \mathrm{NH}_{4} \mathrm{Cl},{ }^{475} \quad$ Silica/Fe (Ferrihydrite silica aerogels), ${ }^{476} \mathrm{Si}-\mathrm{MCM}-41$ supported metal halides/MWI, ${ }^{477}$ $\mathrm{H}_{3} \mathrm{PW}_{12} \mathrm{O}_{40},{ }^{478} \quad \mathrm{InBr}_{3},{ }^{479} \quad \mathrm{Ph}_{3} \mathrm{PH}^{+} \mathrm{ClO}_{4}{ }^{-},{ }^{480} \quad N$-butyl- $N, N$-dimethyl-_phenylethylammonium bromide, ${ }^{481} \mathrm{H}_{3} \mathrm{BO}_{3}$ /glacial acetic acid. ${ }^{482}$

2002

$\mathrm{TsOH},{ }^{483} \mathrm{LiBr},{ }^{484} \quad \mathrm{FeCl}_{3} .6 \mathrm{H}_{2} \mathrm{O} \quad$ or $\quad \mathrm{NiCl}_{2} .6 \mathrm{H}_{2} \mathrm{O},{ }^{485} \mathrm{Yb}(\mathrm{OTf})_{3} / \mathrm{THF}^{486}$

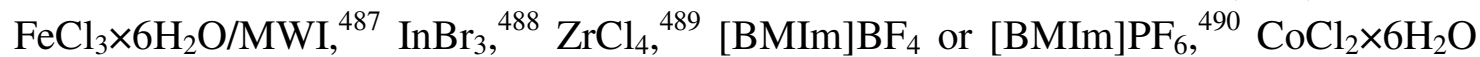
or $\mathrm{LaCl}_{3} \times 7 \mathrm{H}_{2} \mathrm{O} / \mathrm{HCl}^{491}{ }^{491} \mathrm{SnCl}_{2}$ or $\mathrm{CuCl}_{2} \times 2 \mathrm{H}_{2} \mathrm{O}$ or $\mathrm{FeCl}_{3} \times 6 \mathrm{H}_{2} \mathrm{O}$ or $\mathrm{ZnCl}_{2} / \mathrm{HCl} / \mathrm{MWI}{ }^{492}$ $\mathrm{La}(\mathrm{OTf})_{3} / 100{ }^{\circ} \mathrm{C} / \mathrm{sf}^{493} \mathrm{NiCl}_{2} \times 6 \mathrm{H}_{2} \mathrm{O},{ }^{494} \mathrm{InBr}_{3},{ }^{495} \mathrm{Neat} / \mathrm{MWI},{ }^{496}$ PEG-supported/MWI.${ }^{497}$

Over the past two decades or so a large number of publications dealt with catalyst changes rather than any major structural variations (basic skeleton and variations shall be discussed in later section). To discuss all the catalysts individually used so far, does not seem to be 
possible/relevant in this account, so it is decided to present important/salient catalyst developments only.

\subsection{Brønsted acids}

Apart from $\mathrm{HCl}$, many protonic acids like $\mathrm{H}_{2} \mathrm{SO}_{4}$, $p$-toluenesulfonic acid, methanesulfonic acid, $\mathrm{HBF}_{4}$, molybdophosphoric acid, phenylboronic acid, PEG-SO $\mathrm{S}_{3} \mathrm{H}$, boric acid, silica sulfuric acid, acetic acid, formylphenylboronic acid, trifluoro acetic acid, trifluoromethane sulfonic acid etc have been used in this reaction see table 4 .

\subsection{Lewis acids}

These catalysts leave scope for further mechanistic investigations. Authors are leaving to readers to think how fast these catalysts are reported details of each and every paper are omitted in this write-up. It may not the worth while to discuss each and every reagent here in this section it may not be possible to list all because while doing so such papers would exceed four hundered. In Biginelli chemistry Lewis acids halides, triflates and other salts of $\mathrm{Li}, \mathrm{Bi}, \mathrm{Fe}, \mathrm{Cu}, \mathrm{Zn}, \mathrm{Ru}, \mathrm{Rh}, \mathrm{W}$, Mo, Mg, Co, Ni, Sb, Yb, La, Zr, Ce, Sm, Sc, V, Cd, Al, Ag, Nd, Ca, I, B, Sr, Si, Nd, Nb, K, Na, $\mathrm{Ga}, \mathrm{Pb}, \mathrm{Sn}$ etc(sometimes even repeatedly in different journals) have been employed to achieve excellent yields and reduce reaction time as well (Table 4).

\subsection{Ionic liquids}

ILs are considered as the green solvent of present centaury which obey the twelve principles of the green chemistry and are extensively used as catalysts or solvent or both in the organic synthesis. ${ }^{498}$ In this decade the use of ILs in Biginelli reaction have attracted much attention either to enhance rate of reaction or to make synthetic protocol greener. In the synthesis of DHPMs a variety of ILs viz task-specific, ${ }^{263,415}$ Polymer-supported, ${ }^{371}$ chiral ionic liquid ${ }^{197}$ have been used like $[\mathrm{Bmim}]\left[\mathrm{FeCl}_{4}\right],{ }^{223}[\mathrm{Hmim}] \mathrm{HSO}_{4},{ }^{264}\left[\mathrm{C}_{4} \mathrm{mim}\right]\left[\mathrm{HSO}_{4}\right],{ }^{262}[\mathrm{Bmim}] \mathrm{BF}_{4}$ immobilized $\mathrm{Cu}(\mathrm{acac})_{2},{ }^{309}[\mathrm{Bmim}] \mathrm{Sac},{ }^{302,323,392}[\mathrm{Bmim}]\left[\mathrm{PF}_{6}\right],{ }^{490,499}[\mathrm{Bmim}]\left[\mathrm{BF}_{4}\right],{ }^{321,443}[\mathrm{Bmim}] \mathrm{Cl} \times 2 \mathrm{AlCl}_{3},{ }^{414}$ n-butyl pyridinium tetrafluoroborate, ${ }^{354}$ tri-(2-hydroxyethyl) ammonium acetate ${ }^{147}$ etc see Table 4 .

\subsection{Biocatalysts}

Reports on an elegant use of fermenting yeast, ${ }^{250}$ and enzyme, ${ }^{181,500}$ for Biginelli reaction is described see table 4 . Evidently more work is needed in the use of biocatalysts in this reaction.

\subsection{Organocatalysts}

For efficient production of Biginelli compound various organo catalysts like tartaric acid, oxalic acid, citric acid, lactic acid etc are also employed by authors and others. ${ }^{55,62,74,92,114,162,176,212,219}$ 


\section{Rate enhancements}

In the past sonication and presently microwave irradiations are at the forefront of tools employed for time economy and rate enhancement of organic reactions.

\subsection{Sonication}

Though sonication of reaction mixture proved quite fruitful and there is a large amount of literature available on this topic, a detailed discussion on this topic is beyond the scope of this account as it is on general organic chemistry. As far as Biginelli reaction is concerned there are several reports, ${ }^{80,190,201,452,527,530}$ using this technique and along with suitable catalysts systems.

\subsection{Microwave irradiations}

Gedye ${ }^{501}$ introduced the use of a domestic microwave oven in organic reaction in 1986 . There after, there has been very fast investigation of organic reactions employing this technique and the use of microwave in Biginelli reaction is reported by Gupta and co-workers. ${ }^{502}$ As far as our survey is concerned this seems to be first ever application in this reaction. Later on microwave is frequently used in this reaction and more then two dozen protocols are reported employing various solvents/solvent-free and catalysts/catalysts-free for detail see Table 4.

\subsection{Miscellaneous processes}

Other improved techniques used in this reaction are fluorous phase, ${ }^{503}$ solid phase, ${ }^{59,88,101,119,125,149,177,193,504}$ resins, ${ }^{39,110,161,169,253}$ nanoparticles, ${ }^{52,61,87,107,138,240}$ etc.

\section{Biginelli scaffold variations and Biginelli-like reactions}

Variations in the basic scaffold of classical Biginelli structure have attract much attention recently because these structural variations enhance the pharmacological activities of this motif. In this century, the desire for very effective drug molecules which have great pharmacological value, so in this search and to explore synthetic utility in case of Biginelli compound variation have been made at every position of the pyrimidine nucleus from $\mathrm{N}_{1}$ to $\mathrm{C}_{6}$.

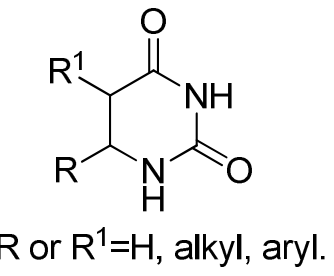

If looked at carefully in this scaffold $\mathrm{C}_{6}$ methyl and $\mathrm{C}_{5}$ substituents are somewhat obstacles in developing the chemistry of this molecule. ${ }^{6(b)}$ Both the substituents are so rigid that these can 
not be readily functionalized or transformed to other function. When these substituents are not there in the well known oxo-analogue 83 of this molecule $\mathrm{C}_{5}-\mathrm{C}_{6}$ bond shows the typical character of enamines and excellent chemistry is developed/being developed on this face. ${ }^{505}$ In this direction authors and others have made considerable efforts to produce $\mathrm{C}_{5}$ unsubstituted compounds. $\mathrm{C}_{5}-\mathrm{C}_{6}$ face of Biginelli compounds has been decorated or altered by employing a variety of acyclic (see Schemes 12-24) and cyclic (see Schemes 25-31) CH-acid components viz ethyl cyanoacetate, 2 -phenylacetophenone, $\beta$-oxodithioesters, dialkyl acetone-1,3-dicarboxylates, ethyl diazoacetate, 5-(4-subtituted phenyl)-5-oxopentanoic acid, enaminone, oxalacetic acid, 2,4dioxo-4-phenylbutanoate, acetophenone derivatives, 5,5-dimethyl-1,3-cyclohexanedione, 3,4dihydro-(2H)-pyran, 1-methyl-1H-pyrrol-2(3H)-one 1, 1-methylpiperidin-2-one 2, 1methylindolin-2-one 3, or 1,3-dimethyl-dihydropyrimidine-2,4-dione, cycloalkanones etc., see Schemes 12-30.<smiles>[R]OO[R10]([H])([H])O[Na]</smiles><smiles>[R]c1ccc(-c2cc(Br)nc(=O)[nH]2)cc1</smiles>

Scheme $12^{124,126}$<smiles>CCOC(=O)CCC#N</smiles>

Scheme $13^{32 b}$<smiles>CCCC(=O)CC(=S)CC</smiles><smiles>[18CH]C=O</smiles><smiles>NC(N)=O</smiles><smiles>CCOS(=O)(=O)CCCCO</smiles><smiles>CSC(=S)C1=C([Al])NC(=O)NC1Br</smiles>

Scheme $14^{506}$ 


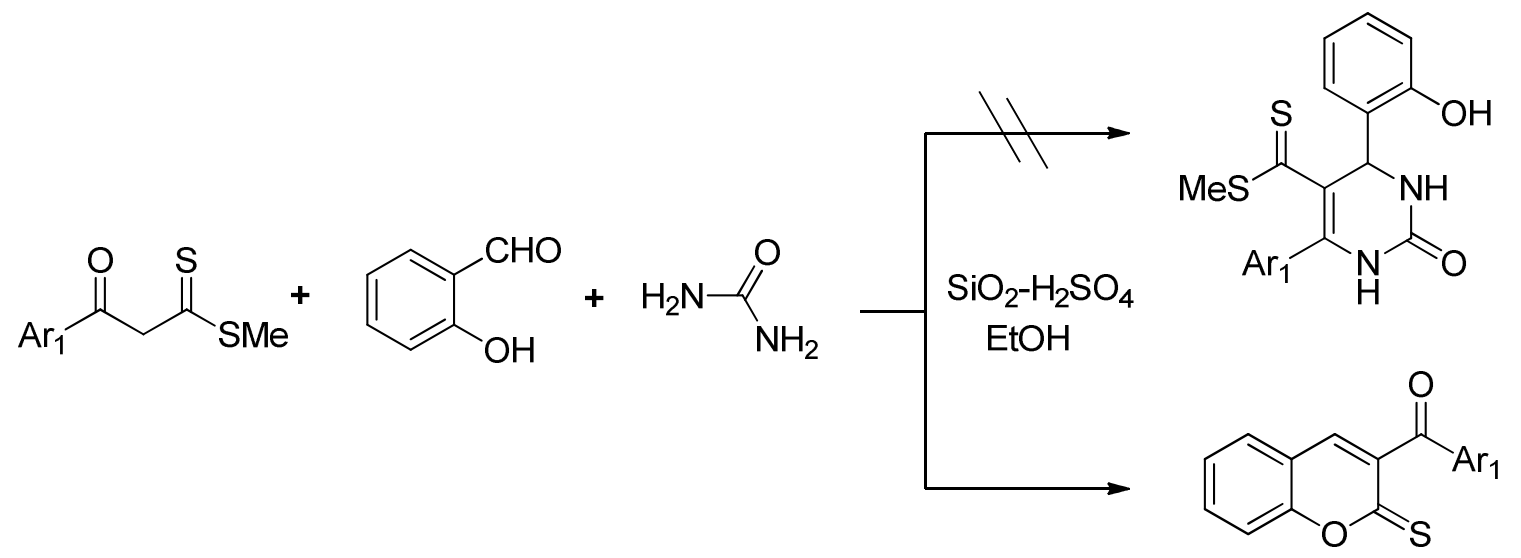

Scheme $\mathbf{1 5}^{506}$

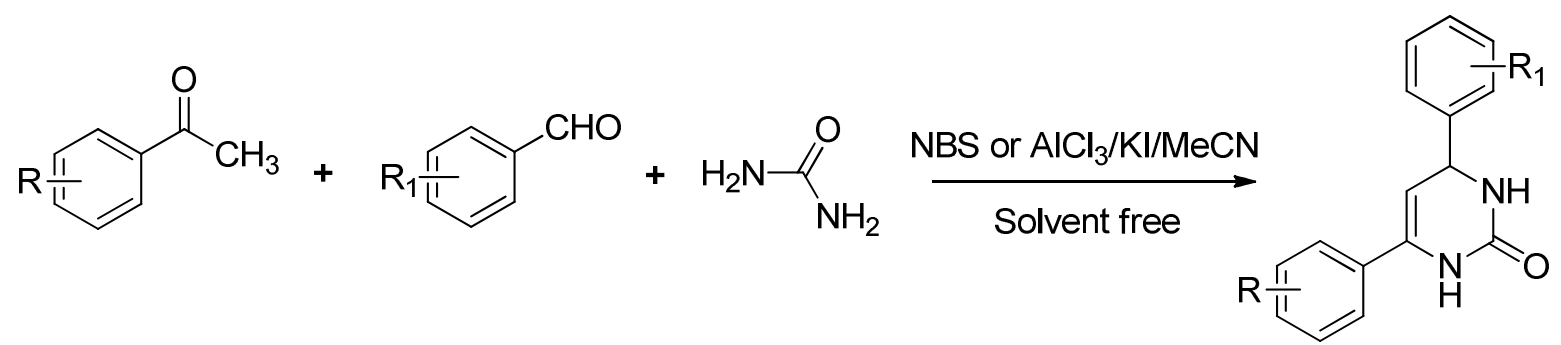

Scheme 16 $^{160,293,507}$<smiles>[R]C(=O)[O+]=[W]</smiles><smiles>[X]C1=NC([R])C(c2ccccc2)=C(c2ccccc2)N1</smiles>

Scheme $\mathbf{1 7}^{19,134}$

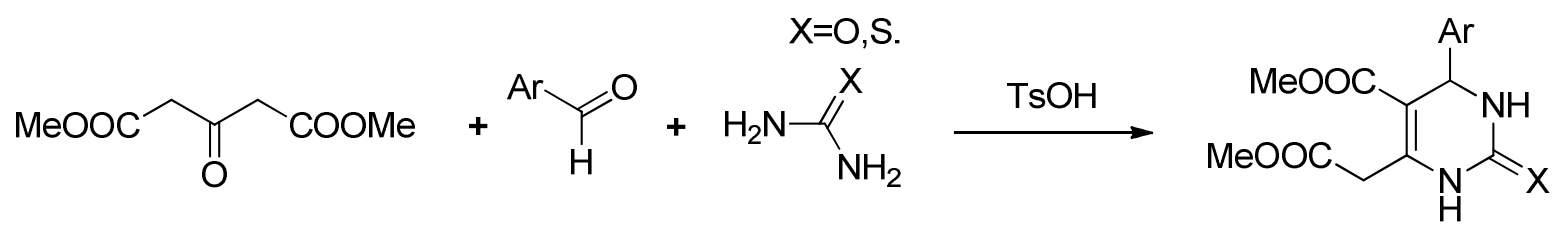

Scheme $18^{194}$

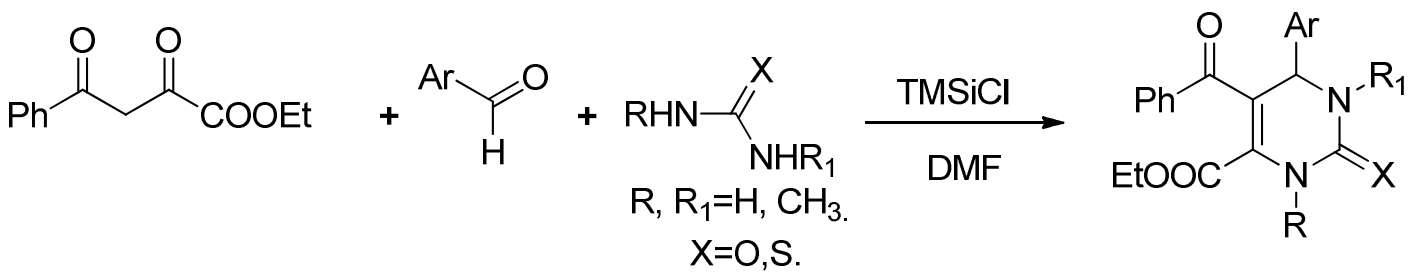

Scheme $19^{64}$ 


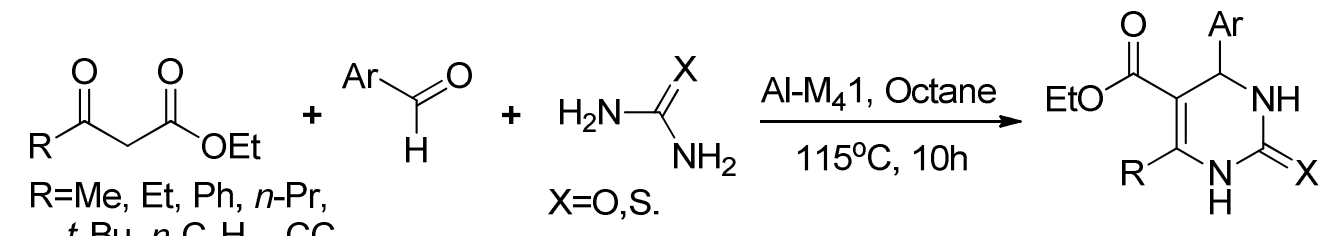
$t$-Bu, $n-\mathrm{C}_{5} \mathrm{H}_{11}-\mathrm{CC}$-, $\mathrm{PhCH}_{2} \mathrm{CH}_{2}$.

Scheme 20 ${ }^{87}$

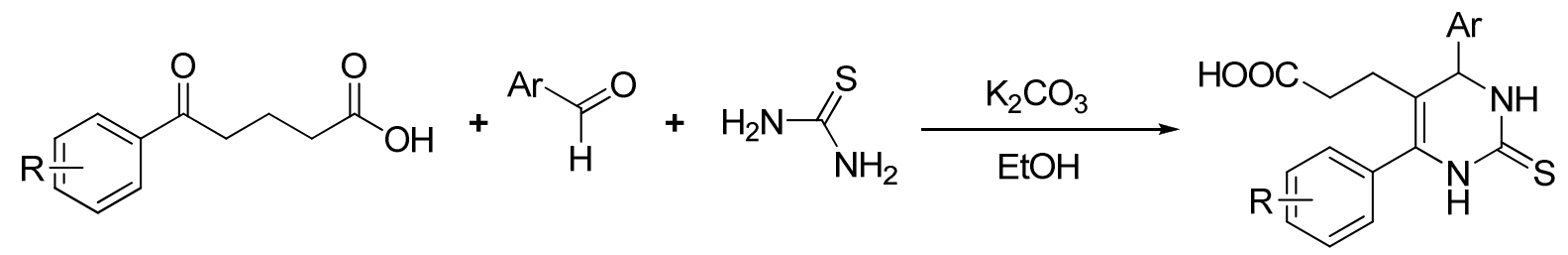

Scheme $21^{30(a)}$<smiles>[X]C1=CNC([X])C(C(=O)c2cc[R17]cc2)C1[Bi]</smiles>

Scheme $22^{170}$<smiles>[R]C(=O)[OH+]Cc1ccc(F)cc1</smiles>

Scheme $23^{196}$

Scheme $24^{305}$

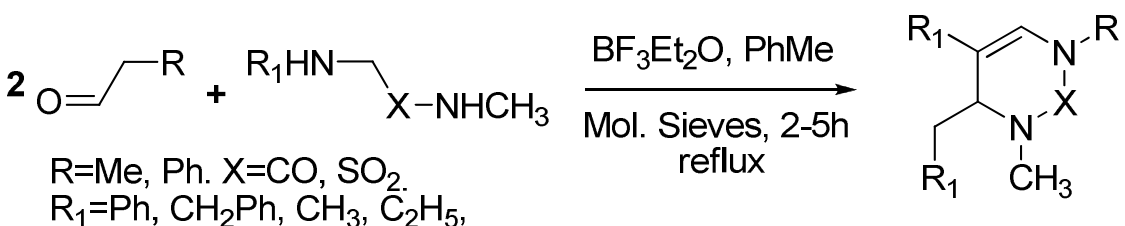

$$
\begin{aligned}
& \begin{array}{c}
\mathrm{C}_{3} \mathrm{H}_{7}, \mathrm{CH}\left(\mathrm{CH}_{3}\right)_{2} \text { etc. }
\end{array}
\end{aligned}
$$

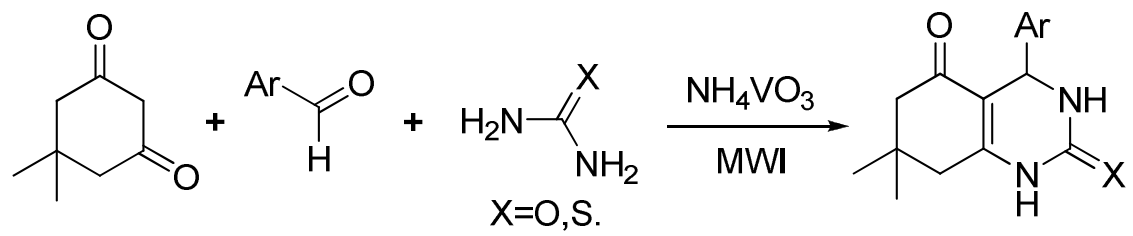

Scheme $\mathbf{2 5}^{66}$ 


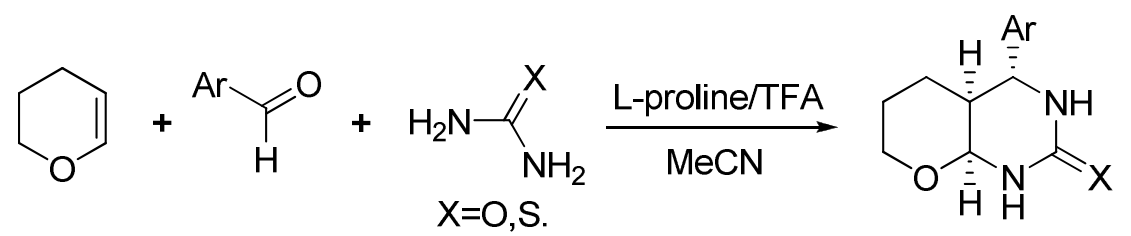

Scheme 26 ${ }^{156,508}$

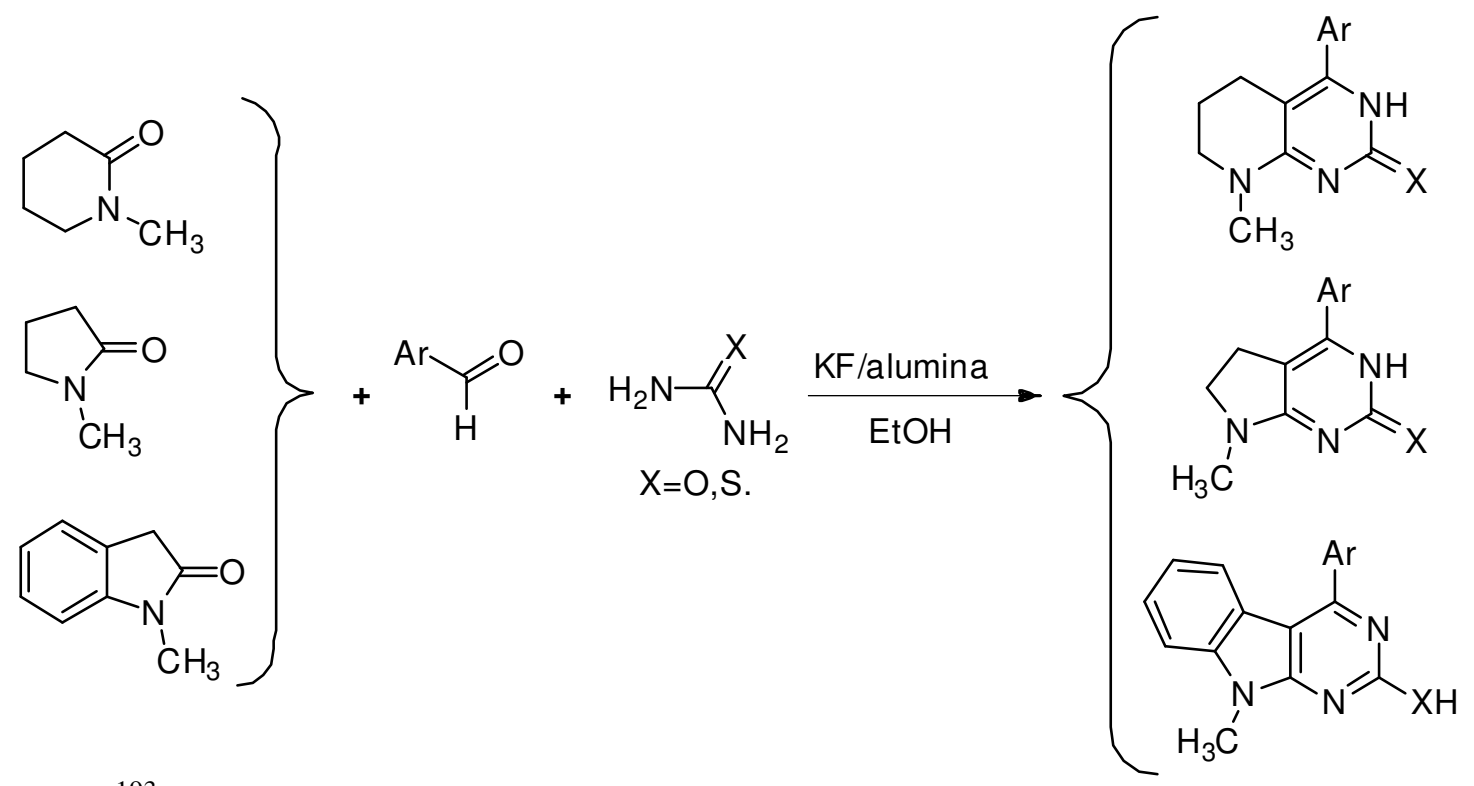

Scheme $27^{193}$

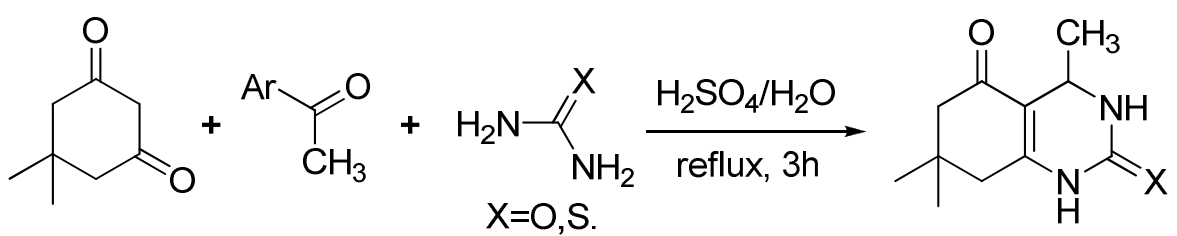

Scheme $28^{341,509}$

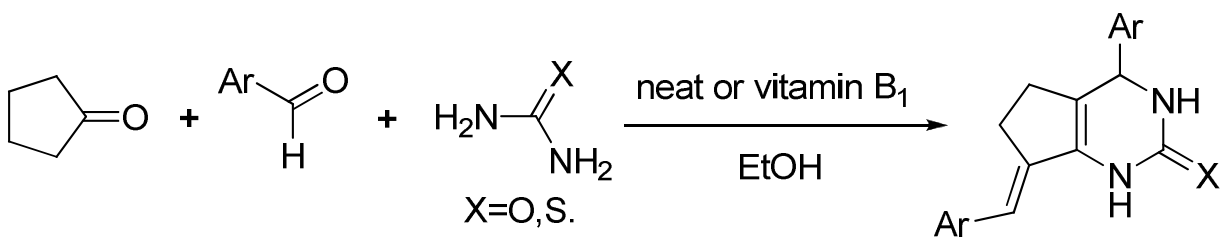

Scheme 29 ${ }^{74,135}$ 

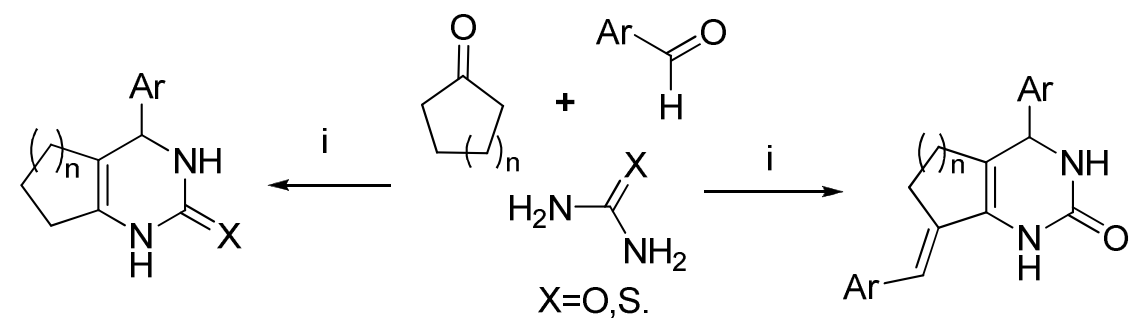

i=TMSCI, DMF/MeCN

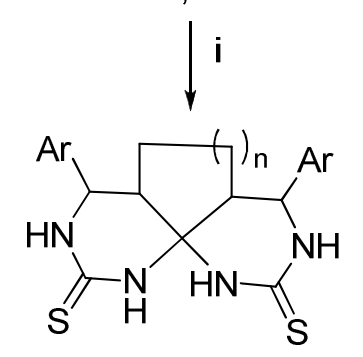

Scheme $30^{367}$

The C-4 position viz. the aldehydic part, has been modified also by different research groups to synthesize Biginelli using an unprotected aldose and 2-methyl-2-phenyl-1,3-oxathiolan-5-one as a mercaptoacetylating active methylene building block with urea/thiourea is reported and which yields diastereoselectively, thiosugar-annulated multifunctionalized dihydropyrimidines via intramolecular domino cyclocondensation reactions of an isolable intermediate Scheme 32. In another case 5-unsubstituted 3,4-dihydropyrimidin-2-ones and thiones having carboxylic group at $\mathrm{C}_{4}$ position achieved by one-pot reaction between oxalacetic acid, thiourea/urea, and aldehyde under microwave irradiation Scheme 33. Similarly 5-chloro/phenoxyl-3-methyl-1phenyl-4-formylpyrazole, steroid-17-ones etc when exchanged with aldehydes used in classical Biginelli corresponding Biginelli like products have been obtained see Scheme 31-37.

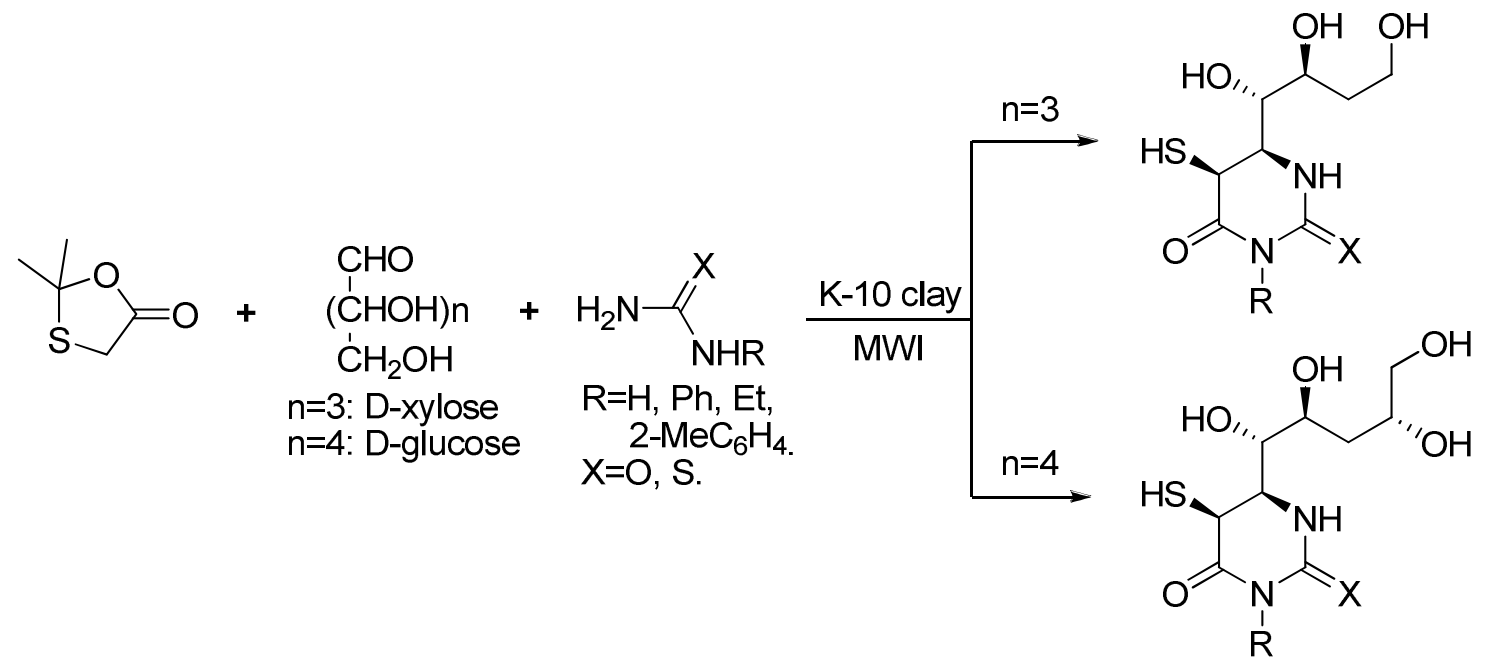

Scheme $31^{306,510}$ 


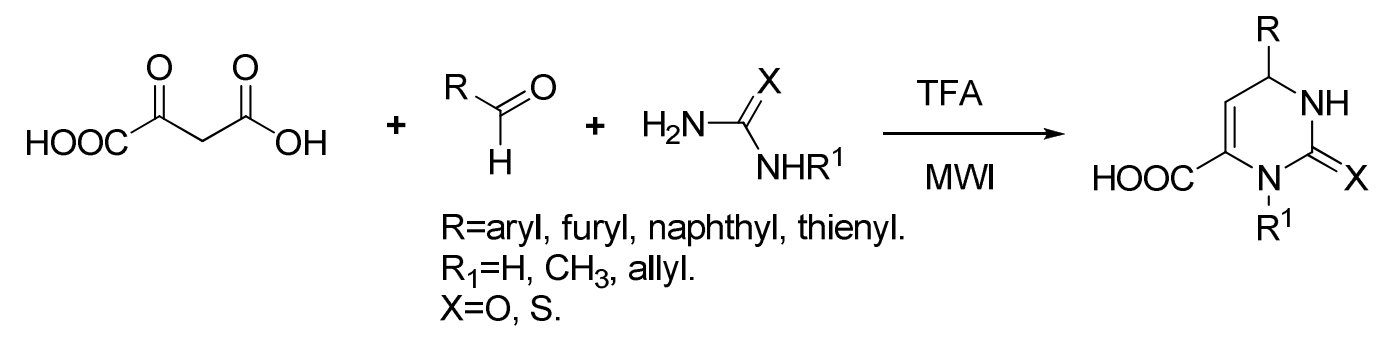

Scheme $32^{48}$<smiles>[R]C(=O)CC(C)=O</smiles><smiles>[R]c1ccc(-c2nn(-c3ccccc3)cc2C=O)cc1</smiles>

Scheme $33^{511}$$$
\mathrm{R}_{1}=\mathrm{H}, \mathrm{CH}_{3} \text {, allyl. }
$$$$
X=O, S \text {. }
$$

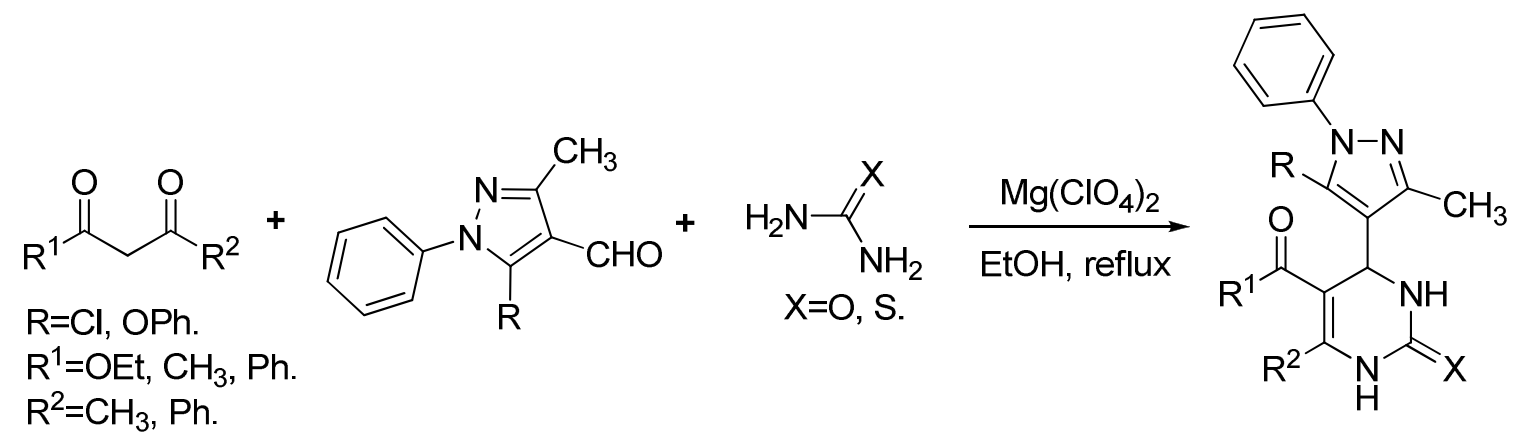

Scheme $34^{360}$

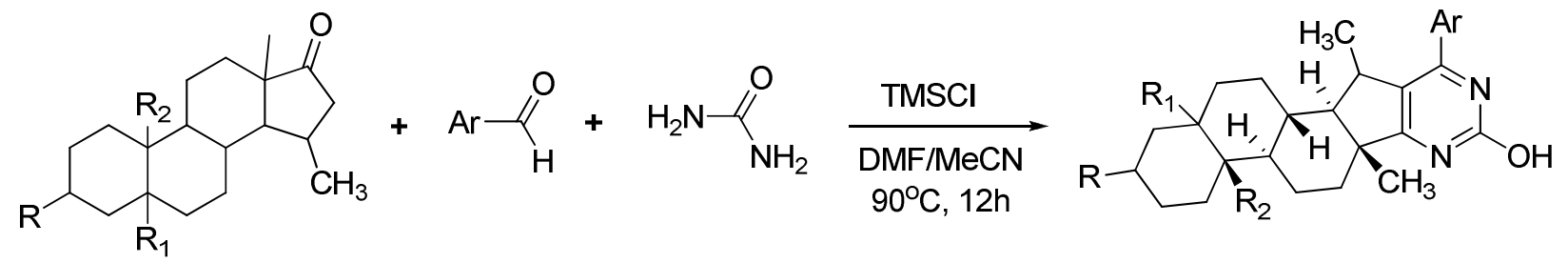

Scheme $35^{512}$ 


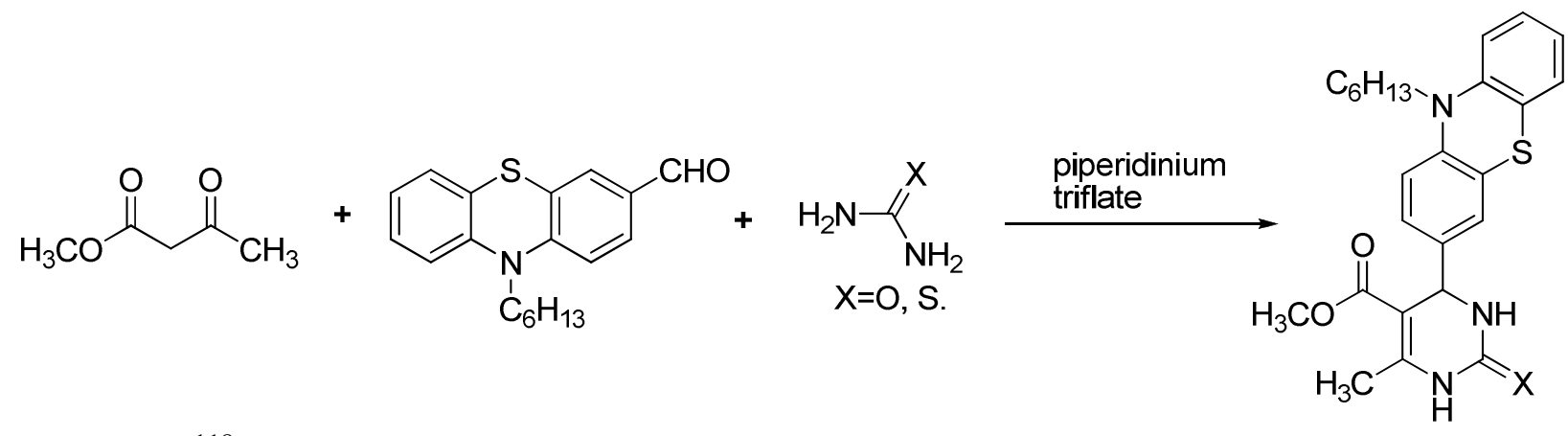

Scheme $36^{118}$<smiles></smiles>

Scheme $37^{118}$

Biginelli-like cyclocondensations based on three-component treatment of 3-amino-1,2,4triazole or 5-aminotetrazole with aldehydes and 1,3-dicarbonyl compounds (see Scheme 38) has been investigated to produce a number of novel derivatives of dihydrotriazolo- and tetrazolopyrimidines. N-Alkoxymethylation of heterocyclic compounds with diethyl phosphite via cleavage of $\mathrm{P}-\mathrm{O}$ bond was investigated and a series of $\mathrm{N}_{3}$-ethoxymethylated heterocyclic compounds have been synthesized Scheme 39. Synthesis of N-3-substituted 3,4dihydropyrimidinones by aza-Michael addition reactions of 3,4-dihydropyrimidinones to $\alpha \beta$ ethylenic compounds catalyzed by $\mathrm{KF} / \mathrm{Al}_{2} \mathrm{O}_{3}$ has been described see Scheme 40.

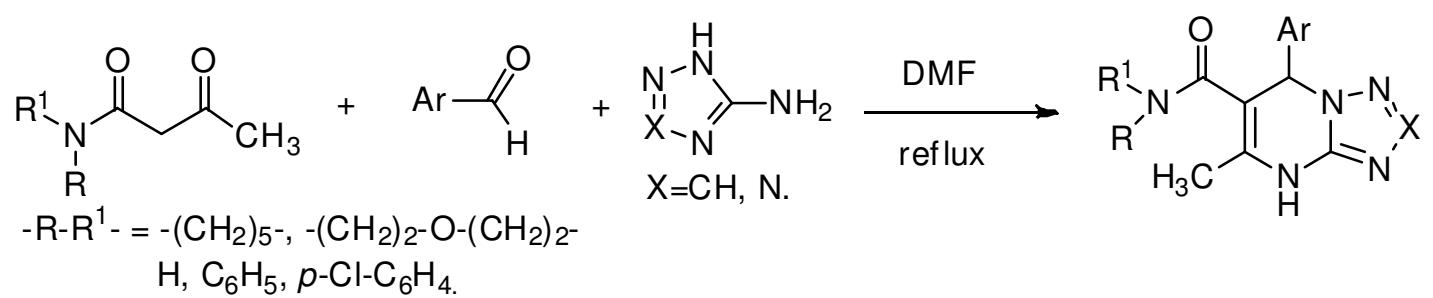

Scheme $38^{513}$ 


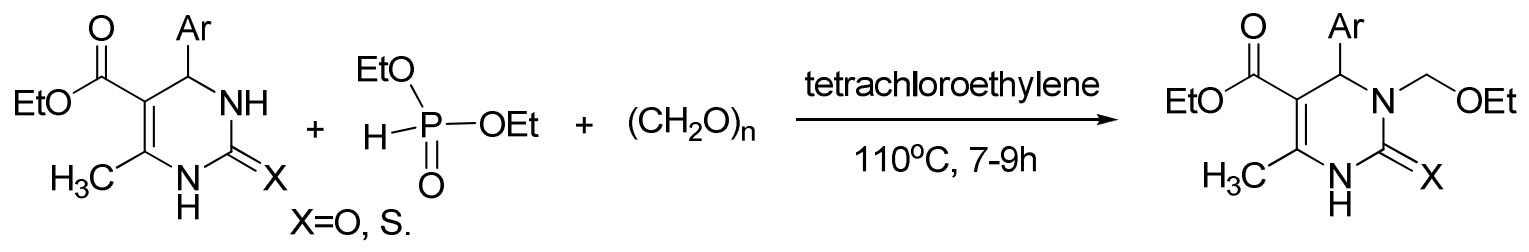

Scheme $39^{514}$<smiles>C=CC(=O)OCCCN(CC)C(=O)C1=C(C)NC(=S)N(CCC(=O)OC)C1Br</smiles>

\section{Scheme $40^{515}$}

Svetlik reported that under traditional Biginelli salicylaldehyde, diethyl acetone-1,3dicarboxylate, and urea can be easily cyclocondensed into compound A, and if diethyl acetone1,3-dicarboxylate is changed to dimethyl acetone-1,3-dicarboxylate, the result compound will be compound B (Scheme 41). PdO catalyzed condensation of salicylaldehyde, ethyl acetoacetate, and urea affords oxygen-bridged pyrimidine tricyclic derivatives in very good yield Scheme 42 which is investigated by Jing.

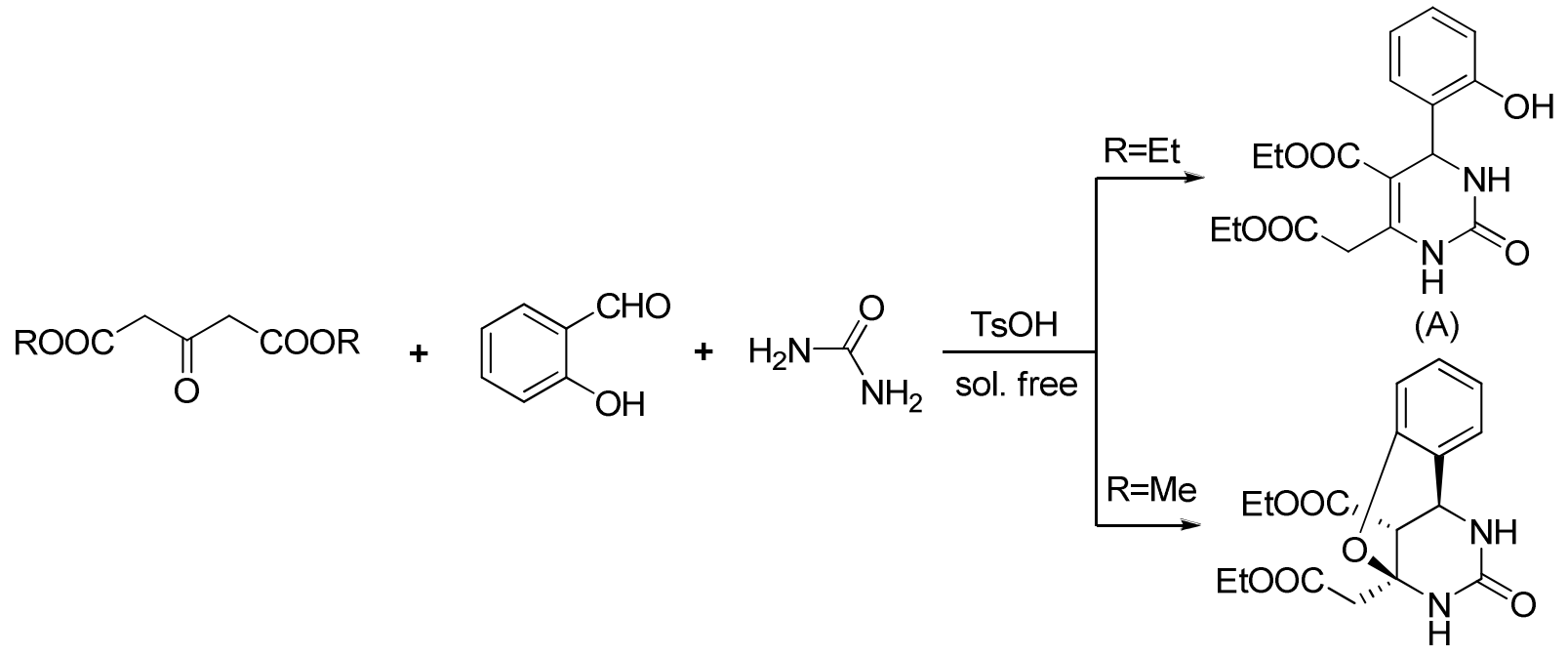

Scheme $41^{194}$

(B) 
<smiles>[R]C(=O)CC(C)=O</smiles>

$\mathrm{R}=\mathrm{OEt}, \mathrm{Me}, \mathrm{OMe}$.<smiles>O=Cc1ccccc1O</smiles><smiles>C=C(N)N</smiles>
$\mathrm{X}=\mathrm{O}, \mathrm{S}$.

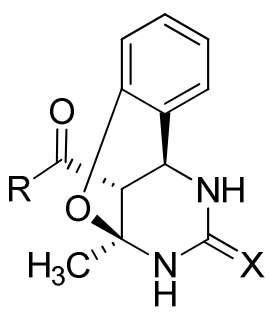

\section{Scheme $42^{168}$}

A one-pot synthesis of bis-1,2,3,4-tetrahydropyrimidine-5-carboxylates is reported via threecomponent condensation of terephthalic aldehyde, 4-chloroacetoacetic ester, and ureas under Biginelli reaction conditions. Interaction of the obtained precursors that contain two highly reactive binucleophilic groups with primary amines leads to bis-heterocyclization with formation of (1,4-phenylene)-bis(pyrrolo[3,4-d]pyrimidine-2,5-diones) Scheme 43. Condensation of diethyl isophthaloyldiacetate, aromatic aldehyde, and urea in the presence of TMSiCl to form 1,3-bis(2oxo- 1,2,3,4-tetrahydropyrimidin-6-yl)benzene derivatives capable to give inclusion complexes with DMF see Scheme 44.<smiles>[R][R][R]([R])=C[R]([R])=[W]</smiles>

Scheme $\mathbf{4 3}^{75,127,516}$

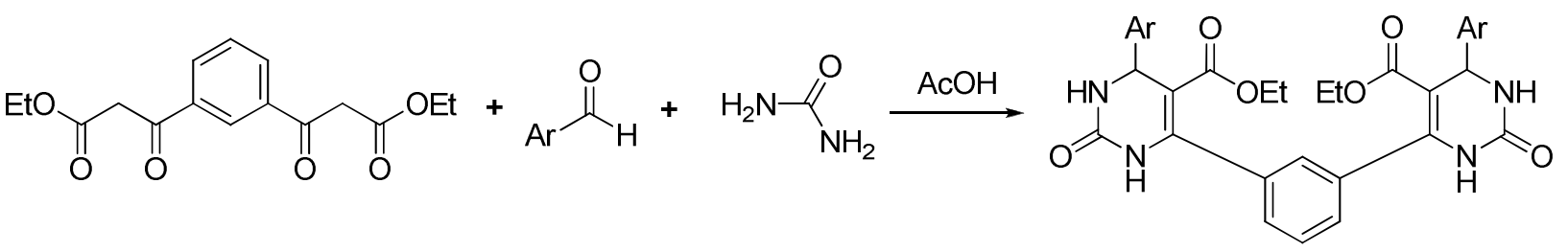

\section{Scheme $44^{517}$}

Spiro-fused Biginelli heterocycles are synthesized by a pseudo four-component reaction of an aldehyde, urea and a cyclic $\beta$-diester or $\alpha \beta$-diamide such as Meldrum acid or barbituric acid derivatives using microwave irradiation under solvent-free conditions in good to high yields 
Scheme 45. Other cyclic $\beta$-keto ester reacts with one molecule of urea and two molecules of aldehyde to give a new family of spiro heterobicyclic aliphatic rings in good yields Scheme 46.<smiles>[X][X]C(=O)CC([X])=O</smiles>

Scheme $\mathbf{4 5}^{38,405,518}$<smiles>[R]C1[X]C(=O)CC1=O</smiles>
$\mathrm{R}=\mathrm{H}, \mathrm{CH}_{3}$, Bn. $\mathrm{R}^{1}=\mathrm{H}$, benzyloxycarbonyl.

Scheme $46^{519}$

A new family of 3,4-dihydropyrimidinones (DHPMs) bearing fluorinated substituents at C-6 have been prepared from gem-difluorinated nitriles, alkyl 3-butenoates and iso(thio)cyanates via tandem nucleophilic addition aza-Michael reaction Schemes 47 and 48. Hetero Diel-Alder reaction on Biginelli substrate is also reported Scheme 49.

Another important report worth mentioning is variation in the $\mathrm{C}_{6}$ methyl group, a development by Kamaljit Singh and co-workers: Scheme 50.

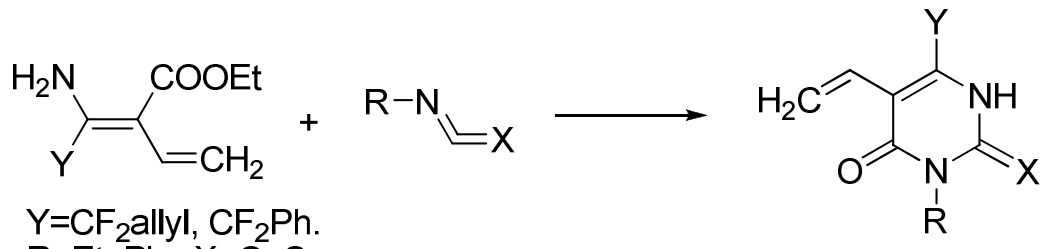

Scheme $47^{520}$

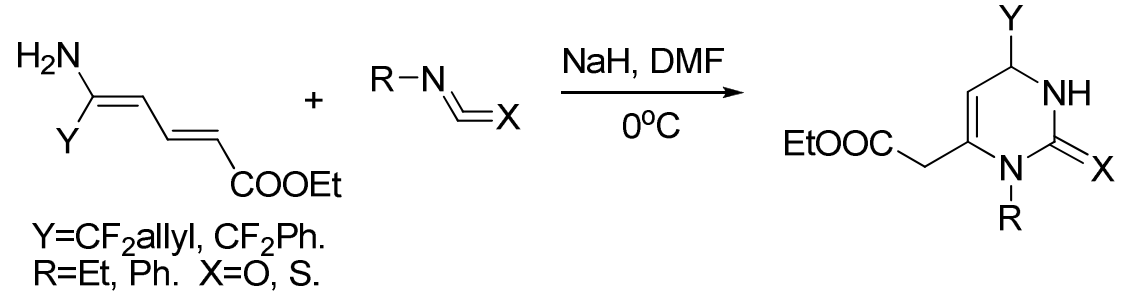

Scheme $48^{151}$ 
<smiles>COC(=O)C1=C(C)NC(=S)NC1c1ccccc1</smiles>

Scheme $49^{521}$

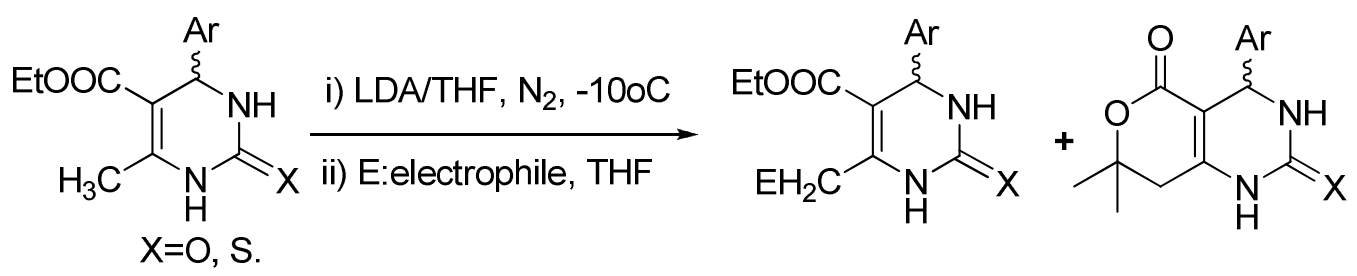

\section{Scheme $50^{522}$}

Unlike the large number of reports that are available for achieving nearly quantitative aromatization of Hantzsch dihydropyridines to pyridines in vitro and in vivo, the structurally similar DHPMs are extremely difficult to oxidize because of their higher oxidation potential compared with that of the dihydropyridines and the sensitivity of the methyl group at C-6 to oxidizing agents. ${ }^{9(\mathrm{a}), 523}$ Dehydrogenation of DHPMs has been attempted employing strong

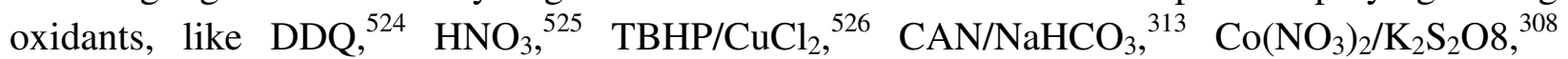

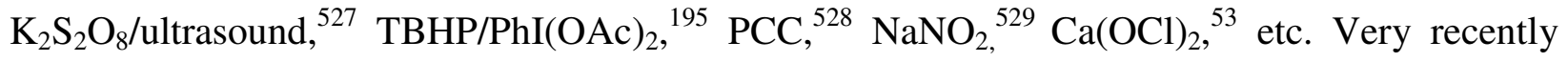
dehydrogenation of various 2-oxo-1,2,3,4-tetrahydropyrimidine-5-carboxamides (THPMs) to 2oxo-1,2-dihydropyrimidine-5-carboxamides (DHPMs) using tetrabutylammonium peroxydisulfate (TBAPS) as an efficient oxidizing agent under thermal and sono-thermal conditions has been investigated, ${ }^{530}$ (Scheme 51) and another report is of a photochemical conversion from 3,4-dihydropyrimidin-2(1H)-ones to pyrimidin-2(1H)-ones at room temperature with visible light irradiation $(\lambda>400 \mathrm{~nm})$ of rhenium(I) complexes $(\mathrm{P} 1-\mathrm{P} 4),{ }^{531}$ see Scheme 52.<smiles>CC1=C(C(=O)[Al])C([18F])([Al])NC(=O)N1</smiles><smiles>Cc1[nH]c(=O)nc(Br)c1C(=O)[Al]</smiles>

Scheme 51 $53,308,530$ 
<smiles>[R]COC(=O)c1c([Al])nc(=O)[nH]c1[R]</smiles>

Scheme $52^{531}$

\section{Asymmetric Biginelli reactions}

During the past few decades there has been intensive researches started to develop methods for producing/ synthesizing one or other of the enantiomers because it is a common observation that individual enantiomers exhibit unlike or even opposite 84-85 biological activities. ${ }^{6(\mathrm{~d}), 6(\mathrm{i})}$ Biginelli compounds contain a stereogenic center, ${ }^{532}$ and the influence of the absolute configuration on the biological activity, ${ }^{373,462,486,523,533}$ has been investigated e.g. in SQ 32926 the $(R)$-enantiomer 86 exhibits $>400$-fold more powerful antihypertensive activity than the $(S)$-isomer, ${ }^{534}$ and the $(S)$ enantiomer of Monastrol 87 has 15-fold more potent anti-cancer activity than $(R)$-Monastrol. ${ }^{535}$ In a similar way, $(S)$-L-771688 (88) is more potent for the treatment of prostatic hyperplasia $(\mathrm{BPH})$ than the $(R)$-enantiomer. ${ }^{35}$ It is also documented that the marine alkaloids i.e. batzelladine $\mathrm{A}$ and $\mathrm{B}$ in enantiomerically pure state have potential anti-HIV activity. ${ }^{22,536}$<smiles>[R20]C(=O)C1=C(C)NC(=O)N[C@H]1[R]</smiles>

(R) Antagonist

84<smiles>[R1]OC(=O)C1=C(C)NC(=O)N[C@H]1[R]</smiles>

(S) Agonist

85<smiles>CC(C)OC(=O)C1=CNC(=O)N(C(N)=O)[C@H]1c1cccc([N+](=O)[O-])c1</smiles>

(R)-SQ 32926

86<smiles>CCOC(=O)C1=C(C)NC(=O)N[C@H]1c1cccc(O)c1</smiles>

(S)-Monastrol

87<smiles>COCC1=C(C(=O)OC)[C@@H](c2ccc(F)c(F)c2)NC(=O)N1</smiles>

(S)-L-771688

88

Therefore, enantio-control in the synthesis of DHPMs has been an objective of primary importance where significant pharmacological activity is concerned. Major achievements of synthetic chemists in the case of DHPMs are discussed here. In this investigation enantiomeric 
pure isomers has been reported employing chiral catalysts, chiral metal complexes, chiral substrate (one of three component viz. aldehydes, urea and active hydrogen component) and enzymatic resolution of a racemic mixture.

Recently, M. A. Blasco et al. reported biocatalytic highly enantioselective synthesis of $(S)$ monastrol ofcourse they used enzymatic resolution employing enzymes lipase from Candida antarctica $\mathrm{B}$ and lipase from Candida rugosa yielding the $(R)$ enantiomer in $48 \%$ yield (66\% ee) and $(S)$ in $31 \%$ yield (97\% ee) Scheme 53. ${ }^{537}$ Optically active DHPMs have also been synthesized through auxiliary assisted asymmetric Biginelli synthesis by Dondoni et al. using chiral starting materials such as $C$-glycosyl substrates 90, 91 and in this investigation the synthesis of two diastereomers of Monastrol analogs bearing the ribofuranosyl moiety has been successfully achieved via formation of diastereomeric $N$-3-ribofuranosyl amides from racemic Monastrol and separation of both diastereomers and subsequent amide hydrolysis of the desired diastereomer: Scheme 54. ${ }^{538}$<smiles>CCCCCCCCC(=O)Oc1cccc(C2NC(=O)NC(C)=C2C(=O)OCC)c1</smiles>

Scheme 53

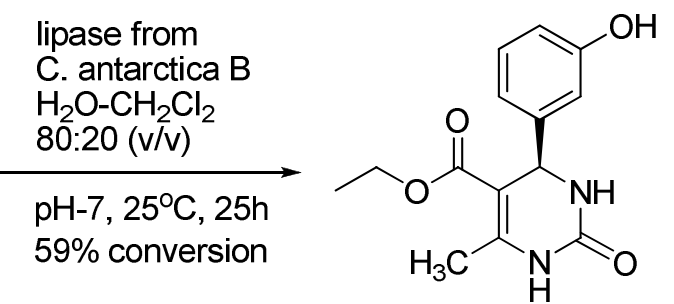

(R) $48 \%$ yield, $66 \%$ ee

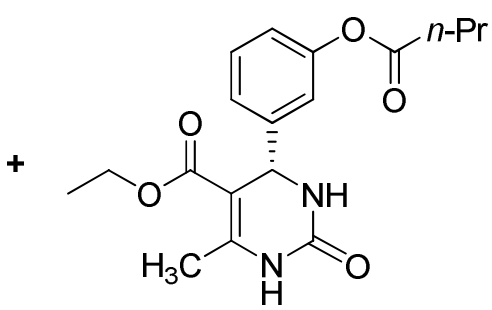

(S) $31 \%$ yield, $97 \%$ ee

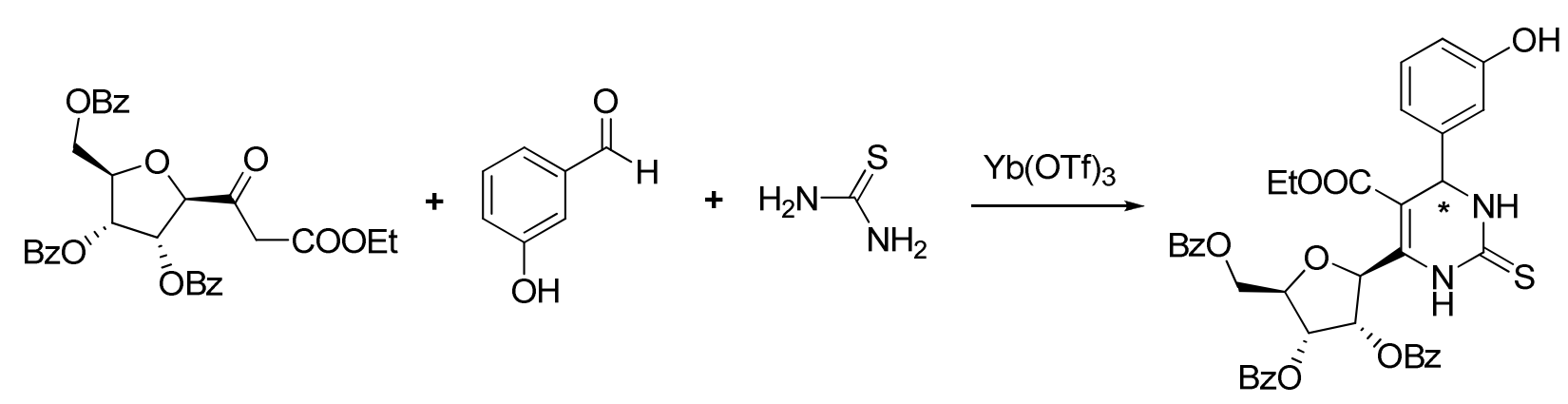

\section{Scheme 54}

Zhu and co-workers reported an enantioselective Biginelli reaction employing a chiral metal catalyst synthesized from ytterbium triflate and a hexadentate ligand 92 which afforded (R)enantiomer of monastrol with $80 \%$ yield and excellent $99 \%$ ee. ${ }^{539}$ Catalytic amount of chiral organocatalyst based on chiral Binaphthol-derived phosphoric acid 93, 97, 98 leading to dihydropyrimidines with enantiomeric excess. ${ }^{540}$ Versatile organocatalytic asymmetric reactions using chiral 5-(pyrrolidin-2-yl)-tetrazole derivatives, trans-4-hydroxyproline derived secondary amine, (2S,4R)-4-tosylamido- $N$-(2,4,6-triphenylbenzene)pyrrolidine-2-carboxamide etc. 89, 94- 
96, 99-102 (Table 5) in combination with achiral Bronsted acid have furnished DHPMs in good yields with excellent (up to $98 \%$ ) enantioselectivity. ${ }^{541}$

Table 5. Use of chiral catalysts and substrate in Biginelli reactions

$R$,<smiles>C[C@H](NC(=O)C1CCCN1)c1ccccc1</smiles>
$\mathrm{R}=\mathrm{H}, \mathrm{OH}$.

89

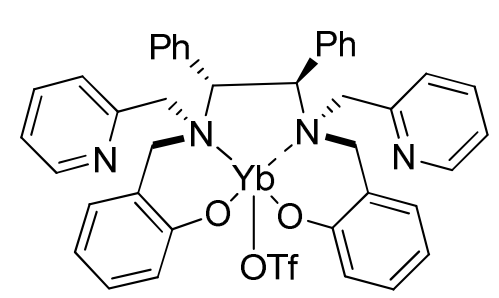

92<smiles>O=C(NC12CC3CC(CC(C3)C1)C2)C1C[C@@H](O)CN1</smiles>

95<smiles></smiles>

98<smiles>CN[C@H]1CNC(C(=O)Nc2ccc(-c3ccccc3)cc2-c2ccccc2)C1</smiles>
101

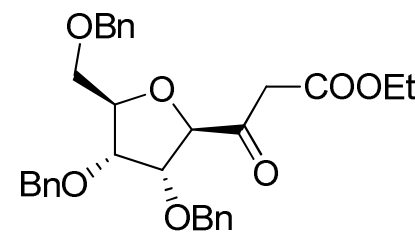

90<smiles>O=P1(O)Oc2c(-c3ccccc3)cc3c(c2-c2c4c(cc(-c5ccccc5)c2OP(=O)(O)O1)CCCC4)CCCC3</smiles>

93<smiles>CC1NCCC2([Si])CNC1C2</smiles>

96<smiles>Cc1ccc([SH](C)(=O)OC2C[C@H](C)CC[C@H]2C(C)C)cc1</smiles>

99

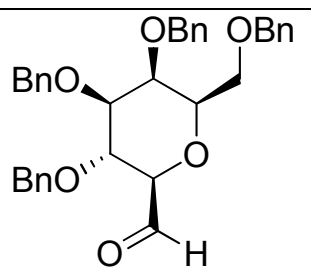

91<smiles>C=CCOC(=O)N[C@H](c1ccc(F)c(F)c1)C(C(C)=O)C(=O)OC</smiles>

94<smiles>O=P1(O)Oc2c([AsH2+])cc3ccccc3c2-c2c(c([AsH2]c3ccccc3)cc3ccccc23)O1</smiles>

97<smiles>CN([As])[C@H]1CN[C@H](c2nnn[nH]2)C1</smiles> 


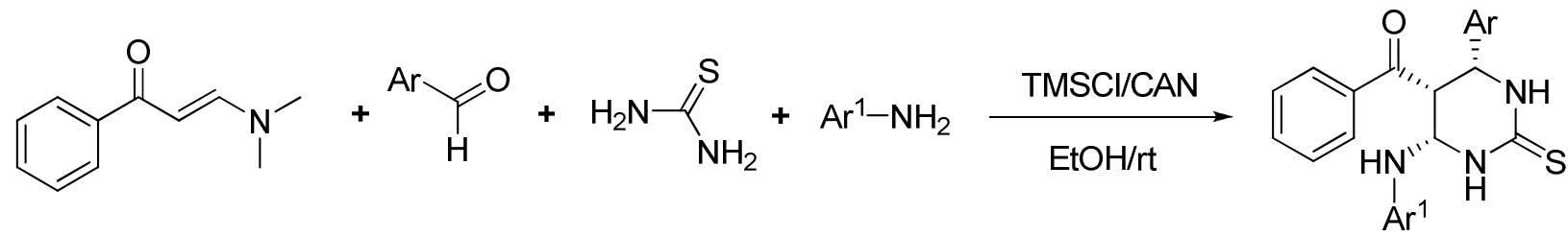

Scheme $55^{542}$

Multicomponent synthesis of tetrahydropyrimidinethiones via condensation four-component aromatic aldehyde, enaminone, aromatic amine, and thiourea also affords products with excellent diastereoselectivity at room temperature: see Scheme $55 .{ }^{542}$

\section{Conclusions; Future Outlook}

As may be seen in this account, initially there was slow activity in this reaction; later it picked up to understand the mechanism etc. In the past two decades or so after the disclosure that this structure is biologically significant, a spate of publications appeared virtually in all less known/regional and reputed international journals of organic chemistry using all types of catalysts variants, basic, acidic Lewis acids simple salts ionic liquids, nano-particles etc, and there are reports describing no catalysts is needed in this reaction. Also for optimizing the production of these compounds techniques like microwave, sonication, etc. are also reported. In these research publications quite often reference is made to C. O. Kappe's mechanism which is based on spectral data without going into actual details. Now some more mechanistic pathways/proposals are advanced which leaves a question mark on the mechanism followed in this reaction at least in the case of Lewis acids used. Certainly in each and every case there can not be single route adopted in this reaction. The real problem in this area was preparation of an optically pure Biginelli scaffold which was achieved recently and further refinements are being actively pursued by several groups. Now biological aspects of these molecules are being examind more intensively and several new activities are being observed, hopefully in near future some molecules in this class may be in clinical use which can lead to real commercial significance to these molecules.

Regarding, further synthetic advancements modification of this scaffold is being attempted and is suitably being tailored to suit the biological needs also there is a large scope of exploring cycloaddition chemistry on this molecule using the different substituents on this or using the double bond available it here is much wider scope of developments. No doubt some scattered efforts is in this direction is already there. We sincerely hope that our suggestions and this account as a whole will stimulate further serious research in this still fertile area. 


\section{Acknowledgements}

The authors are thankful to the Council of Scientific and Industrial Research, New Delhi, India for financial assistance and to the Indian National Science Academy, New Delhi, India for additional financial support for this research project.

\section{References}

1. (a) Hantzsch, A. Ber. 1881, 14, 1637. (b) Hantzsch, A. Justus Liebigs Ann. Chem. 1882, 215,1 .

2. (a) Knoevenagel, E. Ber. 1894, 27, 2345. (b) Knoevenagel, E. Justus Liebigs Ann. Chem. 1894, 281, 25. (c) Knoevenagel E. Chem. Ber. 1896, 29, 172. (d) Knoevenagel, E. Ber. Dtsch. Chem. Ges. 1898, 31, 2585. (e) Knoevenagel, E. Ber. Dtsch. Chem. Ges. 1898, 31, 2596.

3. (a) Biginelli, P. Ber. 1891, 24, 1317. (b) Biginelli, P. Ber. 1891, 24, 2962. (c) Biginelli, P. Gazz. Chim. Ital. 1889, 19, 212.

4. (a) Kenner, G. W.; Todd, A. Pyrimidine and its derivatives. In Heterocyclic Compounds; Elderfield, R. C. Ed.; John Wiley and Sons, Inc.: New York, 1957; Vol. 6, p 239. (b) Zaugg, H. E.; Martin, W. B. Org. Reactions; Adams, R. Ed.; John Wiley and Sons, Inc.: New York, 1965; Vol 14, pp 88-90.

5. Hinkel, L. E.; Hey, D. H. Recl. Trav. Chim. 1929, 48, 1280.

6. Reviews: (a) Biginelli, P. Gazz. Chim. Ital. 1893, 23, 360. (b) Kappe, C. O. Tetrahedron 1993, 49, 6937. (c) Kappe, C. O. Acc. Chem. Res. 2000, 33, 879. (d) Kappe, C. O. Eur. J. Med. Chem. 2000, 35, 1043. (e) Kappe, C. O. QSAR Comb. Sci. 2003, 22, 630. (f) Kappe, C. O.; Stadler, A. Org. React. 2004, 63, 1. (g) Dallinger, D.; Stadler, A.; Kappe, C. O. Pure Appl. Chem. 2004, 76, 1017. (h) Dallinger, D.; Kappe, C. O. Pure Appl. Chem. 2005, 77, 155. (i) Kappe, C. O. In Multicomponent Reactions; Zhu, J., Bienayme, H., Eds.; Wiley- VCH: Weinheim, 2005, p 95. (j) Saini, A.; Kumar, S.; Sandhu, J. S. J. Indian. Chem. Soc. 2007, 84, 959. (k) Vdovina, S. V.; Mamedov, V. A. Russ. Chem. Rev. 2008, 77, 1017. (1) Kolosov, M. A.; Orlov, V. D.; Beloborodov, D. A.; Dotsenko, V. V. Mol. Divers. 2009, 13, 5. (m) Singh, K. D.; Singh, A. K.; Singh, S. Mini-Rev. Med. Chem. 2009, 9, 95. (n) Phucho, I. T.; Nongpiur, A.; Tumtin, S.; Nongrum, R.; Nongkhlaw, R. L. Rasayan J. Chem. 2009, 2, 662. (o) Wan, J-P.; Liu, Y. Synthesis 2010, 23, 3943.

7. Folkers, K.; Johnson, T. B. J. Am. Chem. Soc. 1933, 55, 3784.

8. Sweet, F.; Fissekis, J. D. J. Am. Chem. Soc. 1973, 95, 8741.

9. O’Reilly, B. C.; Atwal, K. S. Heterocycles. 1987, 26, 1185. 
10. Atwal, K. S.; O’Reilly, B. C.; Gougoutas, J. Z.; Malley, M. F. Heterocycles 1987, 26, 1189.

11. Atwal, K. S., Rovnyak, G. C., O’Reilly, B. C., Schwartz, J. J. Org. Chem. 1989, 54, 5898.

12. Kappe, C. O. J. Org. Chem. 1997, 62, 7201.

13. Saloutina, V. I.; Burgarta, Ya. V.; Kuzuevaa, O. G.; Kappe, C. O.; Chupakhin, O. N. J. Fluor. Chem. 2000, 103, 17.

14. Saini, A.; Kumar, S.; Sandhu, J. S. Indian J. Chem. 2007, 46B, 1886.

15. $\mathrm{GaCl}_{3}$ is commercially available in sealed capsules and absorbs moisture as soon as it is exposed to air.

16. Cepanec, I.; Litvic, M.; Filipan-Litvic, M.; Grüngold, I. Tetrahedron 2007, 63, 11822.

17. Ma, J. G.; Zhang, J. M.; Jiang, H. H.; Ma, W. Y.; Zhou, J. H. Chin. Chem. Lett. 2008, 19, 375.

18. De Souza, R. O. M. A.; da Penha, E. T.; Milagre, H. M. S.; Garden, S. J.; Esteves, P. M.; Eberlin, M. N.; Antunes, O. A. C. Chem. Eur. J. 2009, 15, 9799.

19. Shen, Z-L.; Xu, X-P.; Ji, S-J. J. Org. Chem. 2010, 75, 1162.

20. Litvic, M.; Vecenaj, I.; Ladisic, Z. M.; Lovric, M.; Vinkovic, V.; Litvic, M. F. Tetrahedron 2010, 66, 3463.

21. Hentrich, W.; Schepss, W. (FarbenIndustrie, I.G.) D. R. P. 1930, 547,057 [Fortsch. Teerfarbenfabr. Verw. Industriezweige; Friedlander, E., Ed; 1932; Vol. 25, p 2590.]

22. Patil, A. D.; Kumar, N. V.; Kokke, W. C.; Bean, M. F.; Freger, A. J.; Debrossi, C.; Mai, S.; Truneh, A.; Faulkner, D. J.; Carte, B.; Breen, A. L.; Hertzbery, R. P.; Johnson, R. K.; Westley, J. W.; Potts, B. C. M. J. Org. Chem. 1995, 60, 1182.

23. (a) Brown, A. M.; Kunje, D. L.; Yatani, A. Nature 1984, 311, 570. (b) Flaim, S. F.; Zelis, R. Fed. Proc. 1981, 40, 2877.

24. (a) Atwal, K. S.; Rovnyak, G. C.; Schwartz, J.; Moreland, S.; Hedberg, A.; Gougoutas, J. Z.; Malley, M. F.; Floyd, D. M. J. Med. Chem. 1990, 33, 1510. (b) Zorkun, I. S.; Sarac, S.; Celebib, S.; Erolb, K. Bioorg. Med. Chem. 2006, 14, 8582. (c) Chikhale, R. V.; Bhole, R. P.; Khedekar, P. B.; Bhusari, K. P. Eur. J. Med. Chem. 2009, 44, 3645. (d) Alam, O.; Khan, S. A.; Siddiqui, N.; Ahsan, W.; Verma, S. P.; Gilani, S. J. Eur. J. Med. Chem. 2010, 45, 5113. (e) Sehon, C. A.; Wang, G. Z.; Viet, A. Q.; Goodman, K. B.; Dowdell, S. E.; Elkins, P. A.; Semus, S. F.; Evans, C.; Jolivette, L. J.; Kirkpatrick, R. B.; Dul, E.; Khandekar, S. S.; Yi, T. Wright, L. L.; Smith, G. K.; Behm, D. J.; Bentley, R. J. Med. Chem. 2008, 51, 6631.

25. (a) Lloyd, J.; Finlay, H. J.; Atwal, K.; Kover, A.; Prol, J.; Yan, L.; Bhandaru, R.; Vaccaro, W.; Huynh, T.; Huang, C. S.; Conder, M.; Jenkins-West, T.; Sun, H.; Li, D.; Levesque, P. Bioorg. Med. Chem. Lett. 2009, 19, 5469. (b) Vacarro, W.; Huynh, T.; Lloyd, J.; Atwal, K. S.; Finlay, H. J.; Levesque, P. C.; Conder, M. L.; Jenkins-West, T.; Shi, H.; Sun, L. Bioorg. Med. Chem. Lett. 2008, I18, 6381. (c) Lloyd, J.; Finlay, H. J.; Vacarro, W.; Hyunh, T.; Kover, A.; Bhandaru, R.; Yan, L.; Atwal, K.; Conder, M. L.; 
Jenkins-West, T.; Shi, H.; Huang, C.; Li, D.; Sun, H.; Levesque, P. Bioorg. Med. Chem. Lett. 2010, 20, 1436.

26. (a) Klein, E.; DeBonis, S.; Thiede, B.; Skoufias, D. A.; Kozielskib, F.; Lebeaua, L. Bioorg. Med. Chem. 2007, 15, 6474. (b) Kaan, H. Y. K.; Ulaganathan, V.; Rath, O.; Prokopcov, H.; Dallinger, D.; Kappe, C. O.; Kozielski, F. J. Med. Chem. 2010, 53, 5676. (c) Wright, C. M.; Chovatiya, R. J.; Jameson, N. E.; Turner, D. M.; Zhu, G.; Werner, S.; Huryn, D.; Pipas, M.; Billy, J. M.; Day, W.; Wip P.; Brodskya, J. L. Bioorg. Med. Chem. 2008, 16, 3291. (d) Agbaje, O. C.; Fadeyi, O. O.; Fadeyi, S. A.; Myles, L. E.; Okoro, C. O. Bioorg. Med. Chem. Lett. 2011, 21, 989. (e) Kumar, B. R. P.; Sankar, G.; Baig, R. B. N.; Chandrashekaran, S. Eur. J. Med. Chem. 2009, 44, 4192. (f) Ibrahim, D. A.; ElMetwally, A. M. Eur. J. Med. Chem. 2010, 45, 1158.

27. Lewis, R. W.; Mabry, J.; Polisar, J. G.; Eagen, K. P.; Ganem, B.; Hess, G. P. Biochemistry 2010, 49, 4841.

28. Chiang, A. N.; Valderramos, J-C.; Balachandran, R.; Chovatiya, R. J.; Mead, B. P.; Schneider, C.; Bell, S. L.; Klein, M. G.; Huryn, D. M.; Chen, X. S.; Day, B. W.; Fidock, D. A.; Wipf, P.; Brodsky, J. L. Bioorg. Med. Chem. 2009, 17, 1527.

29. Rajanarendar, E.; Reddy, M. N.; Murthy, K. R.; Reddy, K. G.; Raju, S.; Srinivas, M.; Praveen, B.; Rao, M. S. Bioorg. Med. Chem. Lett. 2010, 20, 6052.

30. (a) Mokale, S. N.; Shinde, S. S.; Elgire, R. D.; Sangshetti, J. N.; Shinde, D. B. Bioorg. Med. Chem. Lett. 2010, 20, 4424. (b) Bahekar, S. S.; Shinde, D. B. Bioorg. Med. Chem. Lett. 2004, 14, 1733. (c) Bahekar, S. S.; Shinde, D. B. Acta Pharm. 2003, 53, 223.

31. (a) Trivedi, A. R.; Bhuva, V. R.; Dholariya, B. H.; Dodiya, D. K.; Kataria, V. B.; Shah, V. H. Bioorg. Med. Chem. Lett. 2010, 20, 6100. (b) Virsodia, V.; Pissurlenkar, R. R. S.; Manvar, D.; Dholakia, C.; Adlakha, P.; Shah, A.; Coutinho, E. C. Eur. J. Med. Chem. 2008, 43, 2103.

32. Chitra, S.; Devanathan, D.; Pandiarajan, K. Eur. J. Med. Chem. 2010, 45, 367. (b) Deshmukh, M. B.; Salunkhe, S. M.; Patil, D. R.; Anbhule, P. V. Eur. J. Med. Chem. 2009, 44, 2651 (c) Kidwai, M.; Saxena, S.; Khan, M. K. R.; Thukral, S. S. Eur. J. Med. Chem. 2005, 40, 816. (d) Ashok, M.; Holla, B. S.; Kumari, N. S. Eur. J. Med. Chem. 2007, 42, 380.

33. (a) Stefani, H. A.; Oliveira, C. B.; Almeida, R. B.; Pereira, C. M. P.; Braga, R. C.; Cella, R.; Borges, V. C.; Savegnago, L.; Nogueira, C. W. Eur. J. Med. Chem. 2006, 41, 513. (b) Ismaili, L.; Nadaradjane, A.; Nicod, L.; Guyon, C.; Xicluna, A.; Robert, J-F.; Refouvelet, B. Eur. J.. Med. Chem. 2008, 43, 1270.

34. Singh, B. K.; Mishra, M.; Saxena, N.; Yadav, G. P.; Maulik, P. R.; Sahoo, M. K.; Gaur, R. L.; Murthy, P. K.; Tripathi, R. P. Eur. J. Med. Chem. 2008, 43, 2717.

35. Barrow, J. C.; Nantermet, P. G.; Selnick, H. G.; Glass, K. L.; Rittle, K. E.; Gilbert, K. F.; Steele, T. G.; Homnick, C. F.; Freidinger, R. M.; Ransom, R. W.; Kling, P.; Reiss, D.; Broten, T. P.; Schorn, T. W.; Chang, R. S. L.; O’Malley, S. S.; Olah, T. V.; Ellis, J. D.; 
Barrish, A.; Kassahun, K.; Leppert, P.; Nagarathnam, D.; Forray, C. J. Med. Chem. 2000, 43, 2703.

36. Zhu, X.; Zhao, G.; Zhou, X.; Xu, X.; Xia, G.; Zheng, Z.; Wang, L.; Yang, X.; Li, S. Bioorg. Med. Chem. Lett. 2010, 20, 299.

37. (a) Garima, Srivastava, V. P.; Yadav, L. D. S. Tetrahedron Lett. 2010, 51, 6436. (b) Khosropour, A. R.; Khodaei, M. M.; Beygzadeh, M.; Jokar, M. Heterocycles 2005, 65, 767.

38. Prajapati, D.; Bhuyan, D.; Gohain, M.; Hu, W. Mol. Divers. 2011, 15, 257.

39. Asghari, S.; Tajbakhsh, M.; Kenari, B. J.; Khaksar, S. Chin. Chem. Lett. 2011, 22, 127.

40. Pasunooti, K. K.; Chai, H.; Jensen, C. N.; Gorityala, B. K.; Wang, S.; Liu, X.W. Tetrahedron Lett. 2011, 52, 80.

41. Salim, S. D.; Akamanchi, K. G. Catal. Commun. 2011, in press.

42. Shirini, F.; Zolfigol, M. A.; Albadi, J. Chin. Chem. Lett. 2011, 22, 318.

43. Wu, M.; Yu, J.; Zhao, W.; Wu, J.; Cao, S. J. Fluor. Chem. 2011, 132, 155.

44. Svetlik, J.; Kettmann, V. Tetrahedron Lett. 2011, 52, 1062.

45. Rao, G. B. D.; Acharya, B. N.; Verma, S. K.; Kaushik, M. P.Tetrahedron Lett. 2011, 52, 809.

46. Kumar, P. M.; Kumar, K. S.; Poreddy, S. R.; Mohakhud, P. K.; Mukkanti, K.; Pal, M. Tetrahedron Lett. 2011, 1187.

47. Ren,Y.; Wang, X.; Wang, W.; Li, B.; Shi, Z.; Zhang, W. Tetrahedron Lett. 2011, 52, 192.

48. Fang, Z.; Lam, Y. Tetrahedron. 2011, 67, 1294.

49. Wan, J. P.; Wang, C. Y. Tetrahedron. 2011, 67, 922.

50. Cho, H.; Nishimura,Y.; Yasui,Y.; Kobayashi, S.; Yoshida, S.; Kwon, E.; Yamaguchi, M. Tetrahedron. 2011, 67, 2661.

51. Oskooie, H. A.; Heravi, M. M.; Karimi, N.; Monjezy, M. H. Synth. Commun. 2011, 41, 826.

52. Zhang, L.Y.; Wang, C. H.; Zhou, Li.; Li, P.H.; Zhang, X.Li. Sci. Chin. Chem. 2011, 54, 74.

53. Tamaddon, F.; Razmi, Z. Synth. Commun. 2011, 41, 485.

54. Wang,Y.; Yu, J.; Miao, Z.; Chen, R. Org. Biomol. Chem. 2011, 9, 3050.

55. Gore, S.; Baskaran, S.; Koenig, B. Green. Chem. 2011, 13, 1009.

56. Svetlik, J.; Veizerova, L. Helv. Chim. Acta 2011, 94, 199.

57. Liu, C.; Zhao, X.; Li, Y. J. Het. Chem. 2011, 48, 92.

58. Couto, I.; Tellitu, I.; Domínguez, E. Arkivoc 2011, (ii), 115.

59. Dastmalbaf, M. Z.; Davoodnia, A.; Heravi, M. M.; Hoseini, N. T.; Khojastehnezhad, A.; Zamani, H. A. Bull. Korean Chem. Soc. 2011, 32, 656.

60. Boumoud, T.; Boumoud, B.; Mosset, P.; Debache, A. E- J. Chem. 2011, 8, 312.

61. Mirjalili, B.F.; Bamoniri, A.; Akbari A. J. Iran. Chem. Soc. 2011, 8, 135.

62. Saha, S.; Moorthy, J. N. J. Org. Chem. 2011, 76, 396. 
63. Gorobets, N. Y.; Sedash,Y. V.; Ostras, K. S.; Zaremba, O. V.; Shishkina, S. V.; Baumer, V. N.; Shishkin, O. V.; Kovalenko, S. M.; Desenko, S. M.; Van der Eycken, E. V. Tetrahedron Lett. 2010, 51, 2095.

64. Ryabukhin, S. V.; Plaskon, A. S.; Bondarenko, S. S.; Ostapchuk, E. N.; Grygorenko, O. O.; Shishkin, O. V.; Tolmachev, A. A. Tetrahedron Lett. 2010, 51, 4229.

65. Garima; Srivastava, V. P.; Yadav, L. D. S. Tetrahedron Lett. 2010, 51, 6436.

66. Niralwad, K. S.; Shingate, B. B.; Shingare, M. S. Tetrahedron Lett. 2010, 51, 3616.

67. Zych, A. J.; Wang, H. J.; Sakwa, S. A. Tetrahedron Lett. 2010, 51, 5103.

68. Li, W. J.; Liu, S.; He, P.; Ding, M. W. Tetrahedron 2010, 66, 8151.

69. Shaabani, A.; Seyyedhamzeh, M.; Maleki, A.; Hajishaabanha, F.Tetrahedron 2010, 66, 4040.

70. Ryabukhin, S. V.; Plaskon, A. S.; Boron, S. Y.; Volochnyuk, D. M.; Tolmachev, A. A. Mol. Divers. 2011, 15, 189.

71. Liu, C. J.; Wang, J. D. Molecules 2010, 15, 2087.

72. Mousawi, S. M. A.; Apasery, M. A. E.; Elnagdi, M. H. Molecules 2010, 15, 58.

73. Fang, D.; Zhang, D. Z.; Liu, Z. L. Monatsh. Chem. 2010, 141, 419.

74. $\quad$ Lei, M.; Ma. L.; Hu, L. Monatsh. Chem. 2010, 141, 1005.

75. Lebedyeva, I. O.; Povstyanoy, M. V.; Povstyanoy, V. M.; Panasyuk, O. G.; Guban, E. S. Monatsh. Chem. 2010, 141, 997.

76. Valverde, L.F.; Cedillo, F. D.; Luis, A. C. Monatsh. Chem. 2010, 141,75.

77. Srivastava. R. Catal. Lett. 2010, 139, 17.

78. Kurbanova, M. M. Russ. J. Org. Chem. 2010, 46, 599.

79. Makaev, F.; Styngach, E.; Shargarovskii, V.; Bets, L.; Vlad, L. Russ. J. Org. Chem. 2010, 46,610 .

80. Luo, H. L.; Yang, W.; Li, Y.; Yin, S. F. Chem. Natural Comp. 2010, 46, 412.

81. Prajapati, D.; Bhuyan, D.; Gohain, M.; Hu, W. Mol. Divers. 2011, 15, 257.

82. Abdolali, A.; Sadegh, R. Synthesis. 2010, 4057.

83. Wang, X. C.; Yang, G. J.; Quan, Z. J.; Ji, P. Y.; Liang, J. L.; Ren, R. G. Synlett. 2010, 1657.

84. Wang, M.; Song, Z.; Jiang, H.; Gong. H. Prep. Biochem. Biotechnol. 2010, 40, 101.

85. Abdelmadjid, D.; Wassima, G.; Raouf, B.; Ali, B.; Salah, R.; Bertrand, C. Lett. Org. Chem. 2010, 7, 272.

86. Li, J.; Sun, J.; Su, W. Lett.Org. Chem. 2010, 7, 314.

87. Murata, H.; Ishitani, H.; Iwamoto, M. Org. Biomol. Chem. 2010, 8, 1202.

88. Murata, H.; Ishitani, H.; Iwamoto, M. Phys. Chem. Chem. Phys. 2010, 12, 14452.

89. Sadek, K. U.; Al-Qalaf, F.; Abdelkhalik, M. M.; Elnagdi, M. H. J. Het. Chem. 2010, 47, 284.

90. Cai, Y. F.; Yang, H. M.; Li L.; Jiang, K. Z.; Lai, G. Q.; Jiang J. X.; Xu, L. W. Eur. J. Org. Chem. 2010, 26, 4986.

91. Ding, D.; Zhao, C. G. Eur. J. Org. Chem. 2010, 20, 3802. 
92. Lu, N.; Chen, D.; Zhang, G.; Liu, Q. Int. J. Quant. Chem. 2010, 111, 2031.

93. Gupta, P.; Gupta, S.; Sachar, A.; Kour, D.; Singh, J.; Sharma, R. L. J. Het. Chem. 2010, $47,324$.

94. Li, D.; Mao, H.; An, L.; Zhao, Z.; Zou, J. Chin. J. Chem. 2010, 28, 2025.

95. Rahman, M.; Majee, A.; Hajra, A. J. Het. Chem. 2010, 47, 1230.

96. Darwish, E. S.; Abdelhamid, I. A.; Nasra, M. A.; Gallil, F. M. A.; Fleita, D.H. Helv. Chim. Acta 2010, 93, 1204.

97. Cheng, Q.; Wang, Q.; Xu, X.; Ruan, M.; Yao, H.; Yang, X. J. Het. Chem. 2010, 47, 624.

98. Karade, N. N.; Gampawar, S. V.; Tale, N. P.; Kedar, S. B. J. Het. Chem. 2010, 47, 740.

99. Azizian, J.; Mohammadi, M. K.; Firuzi, O.; Mirza, B.; Miri, R. Chem. Biol. Drug Design. 2010, 75, 375.

100. Kassaee, M. Z.; Masrouri, H.; Movahedi, F.; Mohammadi, R. Helv. Chim. Acta 2010, 93, 261.

101. Kamble, V. T.; Muley, D. B.; Atkore, S. T.; Dakore, S. D. Chin. J. Chem. 2010, 28, 388.

102. Nagawade, R. R.; Shinde, D. B. J. Het. Chem. 2010, 47, 33.

103. Mukhopadhyay, C.; Datta, A. J. Het. Chem. 2010, 47, 136.

104. Singh, V.; Kaur, S.; Ratti, R.; Kad, G. L.; Singh, J. Indian J. Chem. 2010, 49B, 611.

105. Hojati, S. F.; Gholizadeh, M.; Haghdoust, M.; Shafiezadeh, F. Korean Chem. Soc. 2010, 31,3238 .

106. Aridoss, G.; Jeong, Y. T. Bull. Korean Chem. Soc. 2010, 31, 863.

107. Sapkal, S. B.; Shelke, K. F.; Shingate, B. B.; Shingare, M. S. Bull. Korean Chem. Soc. 2010, 31, 351.

108. Ranjith, C.; Srinivasan, G. V.; Vijayan, K. K. Bull. Chem. Soc. Japan 2010, 83, 288.

109. Shirini, F.; Yahyazadeh, A.; Abedini, M.; Langroodi, D. I. Bull. Korean Chem. Soc. 2010, 31, 1715.

110. Wang, Q.; Pei, W. J. Iran. Chem. Soc. 2010, 7, 318.

111. Aghayan, M. M.; Moradi, A.; Bolourtchian, M. J. Iran. Chem. Soc. 2010, 7, 269.

112. Ghassamipour, S.; Sardarian, A. R. J. Iran. Chem. Soc. 2010, 7, 237.

113. Wu, M. S.; He, P.; Zhang, X. Z. S. Afr. J. Chem. 2010, 63, 224.

114. Li, N.; Chen, X. H.; Song, J.; Luo, S. W.; Fan, W.; Gong, L. Z. J. Am. Chem. Soc. 2010, 132, 10953.

115. Tamaddon, F.; Razmi, Z.; Jafari, A. A. Tetrahedron Lett. 2010, 51, 1187.

116. Verma, S.; Jain, S. L.; Sain B. Tetrahedron Lett. 2010, 51, 6897.

117. Mandhane, P. G.; Joshi, R. S.; Nagargoje, D. R.; Gill, C. H. Tetrahedron Lett. 2010, 51, 3138.

118. Ramalingan, C.; Park, S. J.; Lee, I. S.; Kwak, Y.W. Tetrahedron 2010, 66, 2987.

119. Murata, H.; Ishitani, H.; Iwamoto, M.; Phys. Chem. Chem. Phys. 2010, 12, 14452.

120. Nath, J.; Chaudhuri, M. K. Synth. Commun. 2010, 40, 2976.

121. Majd, M. M.; Saidi, K.; Khabazzadeh, H. Phosphorus Sulfur Silicon Relat. Elem. 2010, $185,325$. 
122. Wang, D. C.; Guo, H. M.; Qu, G. R. Synth. Commun. 2010, 40, 1115.

123. Pei, W.; Wang, Q. Synth. Commun. 2010, 40, 1209.

124. Phukan, M.; Kalita, M. K.; Borah, R. Green. Chem. Lett. Rev. 2010, 3, 329.

125. Murata, H.; Ishitani, H.; Iwamoto, M. Org. Biomol. Chem. 2010, 8, 1202.

126. Pourghobadi, Z.; Derikvand, F. Chin. Chem. Lett. 2010, 21, 269.

127. Niknam, K.; Hasaninejad, A.; Arman, M. Chin. Chem. Lett. 2010, 21, 399.

128. Wang, M.; Song, Z.; Jiang, H.; Gong, H. Prep. Biochem. Biotechnol. 2010, 40, 101.

129. Quan, Z. J.; Da, Y. X.; Zhang, Z.; Wang, X. C. Catal. Commun. 2009, 10, 1146.

130. Shobha, D.; Chari, M. A.; Ahn, K.H. Chin. Chem. Lett. 2009, 20, 1059.

131. Pani, M. S.; Arjun, M.; Sridhar, D.; Srinivas, K. T. Chin. Chem. Lett. 2009, $20,909$.

132. Lei, M.; Wu, D-D.; Wei, H-G.; Wang, Y-G. Synth. Commun. 2009, 39, 475.

133. Ramalingam, S.; Kumar, P. Synth. Commun. 2009, 39, 1299.

134. Guo, S- R.; Yuan, Y-Q. Synth. Commun. 2009, 39, 2205.

135. Kefayati, H.; Fakhriyannejad, M.; Mohammadi, A. A. Phosphorus Sulfur Silicon Relat. Elem. 2009, 184, 1796.

136. Zhang, H.; Zhou, Z.; Yao, Z.; Xu, F.; Shen, Q. Tetrahedron Lett. 2009, 50, 1622.

137. Chitra, S.; Pandiarajan, K. Tetrahedron Lett. 2009, 50, 2222.

138. Shobha, D.; Chari, M. A.; Mano, A.; Selvan, S. T.; Mukkanti, K.; Vinu, A. Tetrahedron 2009, 65, 10608.

139. Sharghi, H.; Jokar, M. Synth. Commun. 2009, 39, 958.

140. Gui, J.; Liu, D.; Wang, C.; Lu, F.; Lian, J.; Jiang, H.; Sun, Z. Synth. Commun. 2009, 39, 3436.

141. Shaterian, H. R.; Hosseinian, A.; Ghashang, M. Phosphorus Sulfur Silicon Relat. Elem. 2009, 184, 126.

142. Shaterian, H. R.; Hosseinian, A.; Ghashang, M. Phosphorus Sulfur Silicon Relat. Elem. 2009, 184, 197.

143. Bahrami, K.; Khodaei, M. M.; Farrokhi, A. Synth. Commun. 2009, 39, 1801.

144. Wang, M.; Jiang, H.; Song, Z.; Gong, H. Prep. Biochem. Biotechnol. 2009, 39, 372.

145. Reddy, P. N.; Reddy, Y. T.; Reddy, M. N.; Rajitha, B.; Crooks, P. A. Synth. Commun. 2009, 39, 1257.

146. Suresh; Saini, A.; Kumar D.; Sandhu, J. S. Green. Chem. Lett. Rev. 2009, 2, 29.

147. Chavan, S. S.; Sharma, Y. O.; Degani, M. S. Green. Chem. Lett. Rev. 2009, 2, 175.

148. Zeynizadeh, B.; Dilmaghan, K. A.; Yari, M. Phosphorus Sulfur Silicon Relat. Elem. 2009, 184, 2465.

149. Dilmaghan, K. A.; Zeynizadeh, B.; Yari, M. Phosphorus Sulfur Silicon Relat. Elem. 2009, 184, 1722.

150. Shaterian, H. R.; Hosseinian, A.; Ghashang, M.; Khorami, F.; Karimpoor, N. Phosphorus Sulfur Silicon Relat. Elem. 2009, 184, 2333.

151. Fustero, S.; Catalan, S.; Acena, J. L.; Pozo, C. D. J. Fluor. Chem. 2009, 130, 1145.

152. Singh, O. M.; Devi, N. S. J. Org. Chem. 2009, 74, 3141. 
153. Ahmed, B.; Khan, R. A.; Habibullah; Keshari, M. Tetrahedron Lett. 2009, 50, 2889.

154. Singh, K.; Singh, K. Tetrahedron Lett. 2009, 50, 2219.

155. Matache, M.; Dobrota, C.; Bogdan, N. D.; Dumitru, I.; Ruta, L. L.; Paraschivescu, C.C.; Farcasanu, I. C.; Baciu, I.; Funeriu, D. P. Tetrahedron 2009, 65, 5949.

156. Pandey, J.; Anand, N.; Tripathi, R. P. Tetrahedron 2009, 65, 9350.

157. Liu, C. J.; Wang, J. D. Molecules 2009, 14, 763

158. Pan, N.; Zhang, W.; Liu, Q. Molbank 2009, 4, 645.

159. Memarian, H. R.; Farhadi, A. Monatsh. Chem. 2009, 140, 1217.

160. Ren, Y. M.; Cai, C. Monatsh. Chem. 2009, 140, 49.

161. Chandak, H. S.; Lad, N. P.; Upare, P. P. Catal. Lett. 2009, 131, 469.

162. Savant, M. M.; Pansuriya, A. M.; Bhuva, C. V.; Kapuriya, N. P.; Naliapara, Y. T. Catal. Lett. 2009, 132, 281.

163. Sedova, V. F.; Krivopalov, V. P.; Shkurko, O. P. Russ. J. Org. Chem. 2009, 45, 1535.

164. Khrustalev, D. P.; Russ. J. Gen. Chem. 2009, 79, 164.

165. Alibeik, M. A.; Zaghaghi, Z. Chem. Papers. 2009, 63, 97.

166. Horacio, C.; Alexandre, B. D.; Romain, N.; Frank, K.; Bernard, R.; Roman, L. Synlett 2009, 11, 1737.

167. Sik, K. S.; Seung, C. B.; Hoon, L. J.; Kon, L. K.; Hee, L. T.; Ho, K. Y.; Hyunik, S. Synlett 2009, 599.

168. Jing, X.; Li, Z.; Pan, X.; Wang, Q.; Yan, C.; Zhu, H. Synth. Commun. 2009, 39, 3796.

169. Pansuriya, A. M.; Savant, M. M.; Bhuva, C. V.; Kapuriya, N.; Singh, J.; Naliapara, Y. T. Lett. Org. Chem. 2009, 6, 619.

170. Wan, J-P.; Pan, Y-J. Chem. Commun. 2009, 2768.

171. Sohn, J. H.; Choi, H. M.; Lee, S.; Joung, S.; Lee, H. Y. Eur. J. Org. Chem. 2009, 23, 3858.

172. Wang, Y.; Yang, H.; Yu, J.; Miao, Z.; Chen, R. Adv. Synth. Catal. 2009, 351, 3057.

173. Hong, M.; Cai, C.; J. Het. Chem. 2009, 46, 1430.

174. Zalavadiya, P.; Tala, S.; Akbari, J.; Joshi, H. Arch. Pharm. 2009, 342, 469.

175. Valizadeh, H.; Shockravi, A. Heteroatom. Chem. 2009, 20, 284.

176. Wu, Y. Y.; Chai, Z.; Liu, X. Y.; Zhao, G.; Wang, S. W. Eur. J. Org. Chem. 2009, 6, 904.

177. Arjun, M.; Sridhar, D.; Chari, M. A.; Sarangapani, M. J. Het. Chem. 2009, 46, 119.

178. Pansuriya, A. M.; Savant, M. M.; Bhuva, C. V.; Singh, J.; Naliapara, Y. T. Arkivoc 2009, (vii), 79.

179. Kundu, S. K.; Majee, A.; Hajra, A. Indian J. Chem. 2009, 48B, 408.

180. Ramatchandiran, N.; Sumathi, S.; Buvaneswari, G. Indian J. Chem. 2009, 48B, 865.

181. Prasad, A. K.; Arya, P.; Bhatia, S.; Sharma, R. K.; Singh, R.; Singh, B. K.; Eycken, E. V.; Singh, R.; Olsen, C. E.; Parmar, V. S. Indian J. Chem. 2009, 48B, 1738.

182. Hekmatshoar, R.; Heidari, M.; Heravi, M. M.; Baghernejad, B. Bull. Chem. Soc. Ethiop. 2009, 23, 141. 
183. Mase, N.; Nakamura, D.; Kawano, Y.; Suzuki, Y.; Takabe, K. Heterocycles 2009, 78, 3023.

184. Hekmatshoar, R.; Heidari, M.; Heravi, M. M.; Baghernejad, B. J. Korean. Chem. Soc. 2009, 53, 90 .

185. Jing, X.; Li, Z.; Pan, X.; Shi, Y.; Yan, C. J. Iran. Chem. Soc. 2009, 6, 514.

186. Li, N.; Chen, X. H.; Song, J.; Luo, S. W.; Fan, W.; Gong, L. Z. J. Am. Chem. Soc. 2009, $131,15301$.

187. Khaleghi, S.; Heravi, M. M.; Khosroshahi, M.; Behbahani, F. K.; Daroogheha, Z. Green Chem. Lett. Rev. 2008, 1, 133.

188. Mahdavinia, G. H.; Sepehrian, H. Chin. Chem. Lett. 2008, 19, 1435.

189. Zhan, H. W.; Wang, J. X.; Wang, X. T. Chin. Chem. Lett. 2008, 19, 1183.

190. Kumar, H.; Parmar, A. Ultrason. Sonochem. 2008, 15, 129.

191. Palekar, V. S.; Shukla, S. R. Green. Chem. Lett. Rev. 2008, 1, 185.

192. Akbas, E.; Aslanoglu, F. Phosphorus Sulfur Silicon Relat. Elem. 2008, 183, 82.

193. Mizar, P.; Myrboh, B. Tetrahedron Lett. 2008, 49, 5283.

194. Svetlik, J.; Veizerova, L.; Kettmann, V. Tetrahedron Lett. 2008, 49, 3520.

195. Karade, N. N.; Gampawar, S. V.; Kondre, J. M.; Tiwari, G. B. Tetrahedron Lett. 2008, 49, 6698.

196. Schmidt, R. J.; Lombardo, L. J.; Traeger, S. C.; Williams, D. K. Tetrahedron Lett. 2008, 49, 3009.

197. Yadav, L. D. S.; Rai, A.; Rai, V. K.; Awasthi C. Tetrahedron 2008, 64, 1420.

198. Legeay, J. C.; Eynde, J. J. V.; Bazureau, J. P. Tetrahedron 2008, 64, 5328.

199. Hulme, R.; Zamora, O. D. P.; Mota, E. J.; Pasten, M. A.; Rojas, R. C.; Miranda, R.; Hernández, I. V.; Basurto, J. C.; Ferrara, J. T.; Delgado, F. Tetrahedron 2008, 64, 3372.

200. Glasnov T.N.; Tye, H.; Kappe, C. O. Tetrahedron 2008, 64, 2035.

201. Sakhno, Y. I.; Desenko, S. M.; Shishkina, S. V.; Shishkin, O. V.; Sysoyev, D. O.; Groth, U.; Kappe, C. O.; Chebanov, V. A. Tetrahedron 2008, 64, 1104.

202. Heravi, M. M.; Ranjbar, L.; Derikvand, F.; Alimadadi, B. Mol. Divers. 2008, 12, 191.

203. Vdovina, S. V.; Mustakimova, L. V.; Mamedov, V. A. Russ. J. Org. Chem. 2008, 44, 634.

204. Zendehdel, M.; Mobinikhaledi, A.; Asgari, A. J. Inclus. Phenom. Macrocycl. Chem. 2008, 60, 353.

205. Song, D.; Wang, R.; Chen, Y.; Zhang, S.; Liu, C. React. Kinet. Catal. Lett. 2008, 95, 385.

206. Lannou, M-I.; Helion, F.; Namy, J-L. Synlett 2008, 105.

207. Wang, M.; Song, Z.; Gong, H.; Jiang, H. Prep. Biochem. Biotechnol. 2008, 38, 105.

208. Xin, J.; Chang, L.; Hou, Z.; Shang, D.; Liu X.; Feng, X. Chem.- A Eur. J. 2008, 14, 3177.

209. Heravi, M. M.; Behbahani, F. K.; Oskooie, H. A. Chin. J. Chem. 2008, 26, 2203.

210. Wang, L.; Cai, C. J. Het. Chem. 2008, 45, 1771.

211. Shobha, D.; Chari, M. A.; Sadanandam, P.; Mukkanti, K. J. Het. Chem. 2008, 45, 1225.

212. Sangshetti, J. N.; Shinde D. B.; Nagnnath, D. J. Het. Chem. 2008, 45, 1191. 
213. Akbal, E.; Aslanoglu, F.; Sener, A.; Anil, B. J. Het. Chem. 2008, 45, 1457.

214. Nasr-Esfahani, M.; Karami, B.; Montazerozohori, M.; Abdi, K. J. Het. Chem. 2008, 45, 1183.

215. Hu, Y.; Lu, X.; Xue, N. J. Het. Chem. 2008, 45, 1095.

216. Khunt, R. C.; Akbari, J. D.; Manvar, A. T.; Tala, S. D.; Dhaduk, M. F.; Joshi, H. S.; Shah, A. Arkivoc 2008, (xi), 277.

217. Rameshwar, N.; Parthasarathy, T.; Reddy, A. R. Indin J. Chem. 2008, 47B, 1871.

218. Pathak, V. N.; Gupta, R.; Varshney, B. Indian J. Chem. 2008, 47B, 434.

219. Ramu, E.; Kotra, V.; Bansal, N.; Varala, R.; Adapa, S. R. Rasayan J. Chem. 2008, 1, 188.

220. Boumoud, T.; Boumoud, B.; Rhouati, S.; Belfaitah, A.; Debache, A.; Mosset, P. E-J. Chem. 2008, 5, 688.

221. Akbari, J. D.; Kachhadia, P. K.; Tala, S. D.; Bapodra, A. H.; Dhaduk, M. F.; Joshi, H, S.; Mehta, K. B.; Pathak, S. J. Phosphorus Sulfur Silicon Relat. Elem. 2008, 183, 1911.

222. Decken, A.; Zamora, M. T.; Duguay, D. R.; Vogels, C. M.; Westcott, S. A. Acta Cryst. Sect. E 2008, 64, 929.

223. Chen, X.; Peng, Y. Catal. Lett. 2008, 122, 310.

224. Ryabukhin, S. V.; Plaskon, A. S.; Ostapchuk, E. N.; Volochnyuk, D. M.; Shishkin, O. V.; Tolmachev, A. A. J. Fluor. Chem. 2008, 129, 625.

225. Lannou, M. L.; Hélion, F.; Namy, J. L. Synlett 2008,105.

226. Khabazzadeh, H.; Saidi, K.; Sheibani, H. Bioorg. Med. Chem. Lett. 2008, 18, 278.

227. Besoluk, S.; Kucukislamoglu, M.; Nebioglu, N.; Zengin, M.; Arslan, M. J. Iran. Chem. Soc. 2008, 5, 62 .

228. Shirini, F.; Zolfigol, M. A.; Abri, A. R. J. Iran .Chem. Soc. 2008, 5, 96.

229. Xu, F.; Wang, J. J.; Tian, Y. P. Synth. Commun. 2008, 38, 1299.

230. Suzuki, I.; Iwata, Y.; Takeda, K. Tetrahedron Lett. 2008, 49, 3238.

231. Valizadeh, H.; Gholipur, H.; Zarrebin, R.; Amiri, M.; Sabzi, M. R. Phosphorus Sulfur Silicon Relat. Elem. 2008, 183, 1552.

232. Ramalingan, C.; Kwak, Y. W. Tetrahedron 2008, 64, 5023.

233. Liu, J. H. Contemp. Chem. Ind. 2008, 37, 56.

234. Wang, M.; Song, Z.; Gong, H.; Jiang, H. Prep. Biochem. Biotechnol. 2008, 38, 105.

235. Pathak, V. N.; Gupta, R.; Varshney, B. Indian J. Chem. 2008, 47B, 434.

236. Debache, A.; Amimour, M.; Belfaitah, A.; Rhouati, S.; Carboni, B. Tetrahedron Lett. 2008, 49, 6119 .

237. Khabazzadeh, H.; Saidi, K.; Sheibani, H. Arkivoc 2008, (xv), 34.

238. Shinde, S. V.; Jadhav, W. N.; Lande, M. K.; Gadekar, L. S.; Arbad, B. R.; Kondre, J. M.; Karade, N. N. Catal. Lett. 2008, 125, 57.

239. Maheswara, M.; Oh, S. H.; Kim, K.; Do, J. Y. Bull. Korean Chem. Soc. 2008, 29, 1752.

240. Gopalakrishnan, M.; Sureshkumar, P.; Thanusu, J.; Kanagarajan, V.; Ezhilarasi, M. R. Lett. Org. Chem. 2008, 5, 142. 
241. Boumoud, T.; Boumoud, B.; Rhouati, S.; Belfaitah, A.; Debache, A.; Mosset, P. E- J. Chem. 2008, 5, 688.

242. Subhas Bose, D.; Idrees, M. J. Het. Chem. 2007, 44, 211.

243. Azizian, J.; Mohammadi, A. A.; Kohshari, M.; Karimi, A. R.; Mohammadizadeh, M. R. J. Het. Chem. 2007, 44, 455.

244. Rajanarendar, E.; Ramesh, P.; Mohan, G.; Kalyan Rao, E. J. Het. Chem. 2007, 44, 483.

245. Mobinikhaledi, A.; Forughifar, N.; Safari, J. A.; Amini, E. J. Het. Chem. 2007, 44, 697.

246. Mukhopadhyay, C.; Datta, A.; Banik, B. K. J. Het. Chem. 2007, 44, 979.

247. Polshettiwara, V.; Varma, R. S. Tetrahedron Lett. 2007, 48, 7343.

248. Banik, B. K.; Reddy, A. T.; Datta, A.; Mukhopadhyay, C. Tetrahedron Lett. 2007, 48, 7392.

249. Kumar, A.; Maurya, R. A. Tetrahedron Lett. 2007, 48, 4569.

250. Jiang, C.; You, Q. D. Chin. Chem. Lett. 2007, 18, 647.

251. Gupta, R.; Paul, S.; Gupta, K. J. Mol. Catal. Chem. 2007, 266, 50.

252. Jain, S. L.; Joseph, J. K.; Singhal, S.; Sain, B. J. Mol. Catal. A. Chem. 2007, 268, 134.

253. Lin, H.; Zhao, Q.; Xu, B.; Wang, X. J. Mol. Catal. A. Chem. 2007, 268, 221.

254. Zhao, X. D.; Yu, Y.; Liu, D. J. Liaoning Univ. Petrol. Chem. Technol. 2007, 27, 35.

255. Shi, L.; Zhu, G. H.; Ding, X. Y.; Li, J. H.; Jing, X. H.; Hua, P. Chem. Reagents 2007, 29, 251.

256. Yu, Y.; Liu, D.; Liu, C. S.; Luo, G. X. Chem. Reagents 2007, 29, 181.

257. Pore, D. M.; Desai, U. V.; Thopate, T. S.; Wadgaonkar, P. P. Austral. J. Chem. 2007, 60, 435.

258. Gupta, R.; Gupta, M.; Paul, S.; Gupta, K. Can. J. Chem. 2007, 85, 197.

259. Chen, W. Y.; Qin, S. D.; Jin J. R. Synth. Commun. 2007, 37, 47.

260. Niknam, K.; Daneshvar, N. Heterocycles 2007, 71, 373.

261. Arfan, A.; Paquin, L.; Bazureau, J. P. Russ. J. Org. Chem. 2007, 43, 1058.

262. Dong, F.; Jun, L.; Xinli, Z.; Zhiwen, Y.; Zuliang, L. J. Mol. Catal. A. Chem. 2007, 274, 208.

263. Ma, J. J.; Zang, X. H.; Zhou, X.; Wang, C.; Li, J. C.; Li, Q. Indian J. Chem. 2007, 46B, 2045.

264. Romanelli, G. P.; Sathicq, A. G.; Autino, J. C.; Baronetti, G.; Thomas, H. J. Synth. Commun. 2007, 37, 3907.

265. Yu, Y.; Liu, D.; Liu, C Jiang, H.; Luo, G. Prep. Biochem. Biotechnol. 2007, 37, 381.

266. Ramesha, S.; Bhojya Naik, H. S.; Harish Kumar, H. N. J. Sulfur. Chem. 2007, $28,573$.

267. Rodriguez-Dominguez, J. C.; Bernardi, D.; Kirsch. G. Tetrahedron Lett. 2007, 48, 5777.

268. Nandurkar, N. S.; Bhanushali, M. J.; Bhor, M. D.; Bhanage, B. M. J. Mol. Catal. A. Chem. 2007, 271, 14.

269. Saini, A.; Kumar, S.; Sandhu, J. S. Indian J. Chem. 2007, 46B, 1690.

270. Kumar, A.; Maurya, R. A. J. Mol. Catal. A. Chem. 2007, 272, 53. 
271. Ding, X. Y.; Shi, L.; Jing, X. H.; Zhu, G. H.; Li, J. H.; Hua, P. Chem. Reagents 2007, 29, 175.

272. Zhou, Z.; Han, X.; Xu, F.; Shen, Q.; Zhang, Y. J. Suzhou Univ. Nat. Sci. Ed. 2007, 23, 67

273. Bandgar, B. P.; Kamble, V. T.; Bavikar, S. N.; Dhavane, A. J. Chin. Chem. Soc. 2007, $54,263$.

274. Sharma, S. D.; Gogoi, P.; Konwar, B. Green. Chem. 2007, 9, 153.

275. Jain, S. L.; Singhal, S.; Sain, B. Green. Chem. 2007, 9, 740.

276. Pisani, L.; Prokopcova, H.; Kremsner, J. M.; Kappe, C. O. J. Comb. Chem. 2007, 9, 415.

277. Shirini, F.; Marjani, K.; Nahzomi, H. T. Arkivoc 2007, (i), 51.

278. Karade, H. N.; Sathe, M.; Kaushik, M. P. Molecules 2007, 12, 1341.

279. Ryabukhin, S. V.; Plaskon, A. S.; Ostapchuk, E. N.; Volochnyuk, D. M.; Tolmachev, A. A. Synthesis 2007, 417.

280. Ryabukhin, S. V.; Plaskon, A. S.; Ostapchuk, E. N.; Volochnyuk, D. M.; Shivanyuk, A. N.;Tolmachev, A. A. Org. Lett. 2007, 9, 4215.

281. Yu, Y.; Liu, D.; Liu, C.; Luo, G. Bioorg. Med. Chem. Lett. 2007, 17, 3508.

282. Kalita, H. R.; Phukan, P. Catal. Commun. 2007, 8, 179.

283. Chen, W. Y.; Qin, S. D.; Jin, J. R. Catal. Commun. 2007, 8, 123.

284. Bigdeli, M. A.; Jafari, S.; Mahdavinia, G. H.; Hazarkhani, H. Catal. Commun. 2007, 8, 1641.

285. Maradur, S. P.; Gokavi, G. S. Catal. Commun. 2007, 8, 279.

286. Fu, Y.; Zhang, A. L.; Du, H. M.; Lei, T.T. Chem. Bioeng. 2007, 24, 28.

287. Wang, D. C.; Yuan, T. F.; Yang, X. N.; Qu, G. R.Chin. J. Org. Chem. 2007, 27, 1034.

288. Lin, H. X.; Zhao, Q. J.; Xu, B.; Wang, X. H. Chin. Chem. Lett. 2007, 18, 502.

289. Sancheti, A.; Mehta, S.; Sony, M. D.; Vardia, J.; Punjabi, P. B. Afinidad. 2007, 64, 730.

290. Ahmed, N.; Van Lier, J. E. Tetrahedron Lett. 2007, 48, 5407.

291. Zumpe, F. L.; Flub, M.; Schmitz, K.; Lender, A. Tetrahedron Lett. 2007, 48, 1421.

292. Liang, B.; Wang, X.; Wang, J. X.; Du, Z. Tetrahedron 2007, 63, 1981.

293. Shi, F.; Jia, R.; Zhang, X.; Tu, S.; Yan, S.; Zhang, Y.; Jiang, B.; Zhang, J.; Yao, C. Synthesis 2007, 2782.

294. Deshmukh, M. B.; Anbhule, P. V.; Jadhav, S. D.; Mali, A. R.; Jagtap, S. S.; Deshmukh, S. A. Indian J. Chem. 2007, 46B, 1545.

295. Aslanoglu, F.; Akbas, E.; Sönmez, M.; Anil, B. Phosphorus Sulfur Silicon Relat. Elem. 2007, 182, 1589.

296. Kamal, A.; Krishnaji, T.; Azhar, M. A. Catal. Commun. 2007, 8, 1929.

297. Adibi, H.; Samimi, H. A.; Beygzadeh, M. Catal. Commun. 2007, 8, 2119.

298. Jain, S. L.; Prasad, V. V. D. N.; Sain, B. Catal. Commun. 2007, 9, 499.

299. Niknam, K.; Zolfigol, M. A.; Hossieninejad, Z.; Daneshvar, N. Chin. J. Catal. 2007, 28, 591.

300. Yang, Z. P.; Sheng, S. R.; Lin, S. Y.; Zou, W.; Liu, X. L. J. Jiangxi Norm. Univ. (Nat. Sci. Ed.) 2007, 31, 262. 
301. Wang, S. W.; Cao, W.; Li, M. Res. Explor. Lab. 2007, 26, 100.

302. Russowsky, D.; Benvenutti, E. V.; Roxo, G. S.; Grasel, F. Lett. Org. Chem. 2007, 4, 39.

303. Shaabani, A.; Sarvary, A.; Rahmati, A.; Rezayan, A. H. Lett. Org. Chem. 2007, 4, 68.

304. Bailey, C. D.; Houlden, C. E.; Bar, G. L. J.; Lloyd-Jones, G. C.; Booker-Milburn, K. I. Chem. Commun. 2007, 28, 2932.

305. Yadav, L. D. S. Awasthi, C.; Rai, V. K.; Rai, A. Tetrahedron Lett. 2007, 48, 4899.

306. Legeay, J. C.; Eynde, J. J. V.; Bazureau, J. P. Tetrahedron Lett. 2007, 48, 1063.

307. Shanmugam, P.; Perumal, P. T. Tetrahedron 2007, 63, 666.

308. Jain, S. L.; Joseph, J. K.; Sain, B. Catal. Lett. 2007, 115, 52.

309. Nilsson, B. L.; Overman, L. E. J. Org. Chem. 2006, 71, 7706.

310. Manhas, M. S.; Ganguly, S. N.; Mukherjee, S.; Jain, A. K.; Ajay, K. Tetrahedron Lett. 2006, 47, 2423.

311. Singh, K.; Singh, S. Tetrahedron Lett. 2006, 47, 8143.

312. Shanmugam, P.; Perumal, P.T. Tetrahedron 2006, 62, 9726.

313. Wang, X.; Quan, Z.; Wang, F.; Wang, M.; Zhang, Z.; Li, Z. Synth. Commun. 2006, 36, 451.

314. Kapoor, K. K.; Ganai, B. A.; Kumar, S.; Andotra, C. S. Can. J. Chem. 2006, 84, 433.

315. Kumar, D.; Sundaree, M. S.; Mishra, B. G. Chem. Lett. 2006, 35, 1074.

316. Angeles-Beltran, D.; Lomas-Romero, L.; Lara-Corona, V. H.; Gonzalez-Zamora, E.; Negron-Silva, G. Molecules 2006, 11, 731.

317. Mamedov, V. A.; Mustakimova, L. V.; Gubaidullin, A. T.; Vdovina, S. V.; Litvinov, I. A.; Reznik, V. S. Chem. Heterocycl. Comp. 2006, 42, 1229.

318. El-Hamouly, W. S.; El-Khamry, A. M. A.; Abbas, E. M. H. Indian J. Chem. 2006, 45B, 2091.

319. Jin, T.; Zhao, Y.; Liu, L.; Li, T. Chin. J. Org. Chem. 2006, 26, 975.

320. Putilova, E. S.; Troitskii, N. A.; Zlotin, S. G.; Khudina, O. G.; Burgart, Y. V.; Saloutin, V. I.; Chupakhin, O. N. Russ. J. Org. Chem. 2006, 42, 1392.

321. Zheng, R.; Wang, X.; Xu, H.; Du, O. Synth. Commun. 2006, 361, 503.

322. Li, M.; Guo, W. S.; Wen, L. R.; Li, Y. F.; Yang, H. Z. J. Mol. Catal. A: Chem. 2006, 258, 133.

323. Mukhopadhyay, C.; Datta, A.; Banik, B. K. Heterocycles 2006, 71, 181.

324. Subhas Bose, D.; Venu Chary, M.; Mereyala H. B. Heterocycles 2006, 68, 1217.

325. Kumar, D.; Mishra, B. G.; Rao, V. S. Indian J. Chem. 2006, 46B, 2325.

326. Kantevari, S.; Bantu, R.; Nagarapu, L. Arkivoc 2006, (xvi), 136.

327. Salehi, P.; Dabiri, M.; Khosropour, A. R.; Roozbehniya, P. J. Iran. Chem. Soc. 2006, 3, 98.

328. Adib, M.; Ghanbary, K.; Mostofi, M.; Ganjali, M. R. Molecules 2006, 11, 649.

329. Chen, X.H, Xu, X. Y.; Liu, H.; Cun, L. F.; Gong, L. Z. J. Am. Chem. Soc. 2006, 128, 14802. 
330. Gangadasu, B.; Palaniappan, S.; Amarnath, C. A.; Rao, V. J. J. Appl. Polym. Sci. 2006, $102,1741$.

331. Shirini, F.; Zolfigol, M. A.; Mollarazi, E. Synth. Commun. 2006, 36, 2307.

332. Hegedus, A.; Hell, Z.; Vígh. I. Synth. Commun. 2006, 36, 129.

333. Mabry, J.; Ganem. B. Tetrahedron Lett. 2006, 47, 55.

334. Suzuki, I.; Suzumura, Y.; Takeda, K. Tetrahedron Lett. 2006, 47, 7861.

335. Debache, A.; Boumoud, B.; Amimour, M.; Belfaitah, A.; Rhouati, S.; Carboni, B. Tetrahedron Lett. 2006, 47, 5697.

336. Zhang, M.; Li, Y. Q.; Zhou, M. Y. Chin. J. Chem. 2006, 24, 282.

337. Zhang, M.; Li, Y. Q. Synth. Commun. 2006, 36, 835.

338. Singh, K.; Arora, D.; Singh, S. Tetrahedron Lett. 2006, 47, 4205.

339. Jain, S. L.; Sharma, V.B.; Sain, B. J. Het. Chem. 2006, 43, 777.

340. Hassani, Z.; Islami, M. R.; Kalantari, M. Bioorg. Med. Chem. Lett. 2006, 16, 4479.

341. Zhou, H.; He, M.; Liu, C.; Jiang, H.; Luo, G. Prep. Biochem. Biotechnol. 2006, 36, 375.

342. Wang, X.; Quan, Z.; Wang, F.; Wang, M.; Zhang, Z.; Li, Z. Synth. Commun. 2006, 36, 451.

343. Liu, C. J.; Wang, J. D.; Li, Y. P. J. Mol. Catal. A. Chem. 2006, 258, 367.

344. Heravi, M. M.; Bakhtiari, K.; Bamoharram, F. F. Catal. Commun. 2006, 7, 373.

345. Fazaeli, R.;Tangestaninejad, S.; Aliyan, H.; Moghadam, M. Appl. Catal. A Gen. 2006, 309, 44.

346. Rafiee, E.; Jafari, H. Bioorg. Med. Chem. Lett. 2006, 16, 2463.

347. Amini, M. M.; Shaabani, A.; Bazgir, A. Catal. Commun. 2006, 7, 843.

348. Joseph, J. K.; Jain, S. L.; Sain, B. J. Mol. Catal. A. Chem. 2006, 247, 99.

349. Rafiee, E.; Shahbazi, F. J. Mol. Catal. A. Chem. 2006, 250, 57.

350. Azizian, J.; Mohammadi, A. A.; Karimi, A. R.; Mohammadizadeh, M. R.; Appl. Catal. A Gen. 2006, 300, 85.

351. Wang, C. F.; Jiang, H.; Gong, H.; Wang, M.; Wang, Z. C. Chin J. Org. Chem. 2006, 26, 333.

352. Kotharkar, S. A.; Nagawade, R. R.; Shinde, D. B. Ukr. Bioorg. Acta. 2006, 2, 17.

353. Zhou, M. Y.; Li, Y. Q. J. Jinan Univ. (Nat. Sci. Med. Ed.) 2006, 27, 435.

354. Wang, J. S.; Li, J. T.; Lin, Z. P. Lett. Org. Chem. 2006, 3, 523.

355. Liang, B.; Wang, X. T.; Wang, J. X Chem. Res. and Appl. 2006, 18, 927.

356. Zhou, H. X.; He, M.; Liu, C. S.; Luo, G. X. J. Liaoning Univ. Petrol. Chem. Technol. 2006, 26, 16.

357. Zhou, H. X.; He, M.; Liu, C. S.; Luo, G. X. Chem. Reagents. 2006, 28, 377.

358. Mishra, B. G.; Kumar, D.; Rao, V. S. Catal. Commun. 2006, 7, 457.

359. Zhang, X.; Li, Y.; Liu, C.; Wang, J. J. Mol. Catal. A. Chem. 2006, 253,207.

360. Khosropour, A. R.; Khodaei, M. M.; Beygzadeh, M.; Jokar, M. Heterocycles 2005, 65, 767. 
361. Blacquiere, J. M.; Sicora, O.; Vogels, C. M.; Cuperlovic-Culf, M.; Decken, A.; Ouellette, R. J.; Westcott, S. A. Can. J. Chem. 2005, 83, 2052.

362. Legeay, J. C.; vanden Eynde, J. J.; Bazureau, J. P. Tetrahedron 2005, 61, 12386.

363. Mobinikhaledi, A.; Foroughifar, N.; Ghorbani, A. R. Phosphorus Sulfur Silicon Relat. Elem. 2005, 180, 1713.

364. Nasr-Esfahani, M.; Khosropour, A. R. Bull. Korean. Chem. Soc. 2005, 29, 1331.

365. Tu, S.; Zhu, X.; Fang, F.; Zhang, X.; Zhu, S.; Li, T.; Shi, D.; Wang, X.; Ji, S. Chin. J. Chem. 2005, 23, 596.

366. Zhu, Y.; Huang, S.; Pan, Y. Eur. J. Org. Chem. 2005, 2354.

367. Han, X.; Xu, F.; Luo, Y.; Shen, Q. Eur. J. Org. Chem. 2005, 1500.

368. Bratenko, M. K.; Chornous, V. A.; Vovk, M. V. Russ. J. Org. Chem. 2005, 41, 95.

369. Putilova, E. S.; Kryshtal, G. V.; Zhdankina, G. M.; Troitskii, N. A.; Zlotin, S. G. Russ. J. Org. Chem. 2005, 41,512.

370. Wang, Z.T.; Wang, S. C.; Xu, L. W. Helv. Chim. Acta 2005, 88, 986.

371. Sabitha, G.; Reddy, K. B.; Srinivas, R.; Yadav, J. S. Helv. Chim. Acta 2005, 88, 2996.

372. Huang, Y. J.; Yang, F. Y.; Zhu, C. J. J. Am. Chem. Soc. 2005, 127, 16386.

373. Bhosale, R. S.; Hashmi, A. M.; Zubaidha, P. K.; Dongre, M. K. Heterocycl. Commun. 2005, 11, 399.

374. Pash, M. A.; Ramachandra Swamy, N.; Jayashankara, V. P. Indian J. Chem. 2005, 44B, 823.

375. Thirupathi Reddy, Y.; Narsimha Reddy, P.; Sunil Kumar, B.; Rao, G. V. P.; Rajitha, B. Indian J. Chem. 2005, 44B, 1304.

376. Kumar, S.; Saini, A.; Sandhu, J. S. Indian J. Chem. 2005, 44B, 762.

377. Adharvana Chari, M.; Shobha, D.; Kiran Kumar, T.; Dubey, P. K. Arkivoc 2005, (xv), 74.

378. Subhas Bose, D.; Sudharshan, M.; Chavhan, S. W. Arkivoc 2005, (iii), 228.

379. Palaniappan, S.; John, A. J. Mol. Catal. A. Chem. 2005, 233, 9.

380. Godoi, M. N.; Costenaro, H. S.; Kramer, E.; Machado, P. S.; Doca, M. G. M.; Russowsky, D. Quim. Nova 2005, 28, 1010.

381. De, S. K.; Gibbs, R. A. Synthesis 2005, 1748.

382. Jin, T.S.; Wang, H. X.; Xing, C. Y.; Li, X. L.; Li, T. S. Synth. Commun. 2005, 34, 3009.

383. De, S.K.; Gibbs, R. A. Synth. Commun. 2005, 35, 2645.

384. Zhang, G. L.; Cai, X. H. Synth. Commun. 2005, 35, 829.

385. El Badaoui, H.; Bazi, F.; Tamani, S.; Boulaajaj, S.; Zahouily, M.; Lazrek, H. B.; Sebti, S. Synth. Commun. 2005, 35, 2561.

386. Manjula, A.; Rao, B. V.; Neelakantan, P. Synth. Commun. 2005, 34, 2665.

387. Salehi, P.; Dabiri, M.; Zolfigol, M. A.; Baghbanzadeh, M. Heterocycles 2005, 65, 1177.

388. Nagawade, R. R.; Kotharkar, S. A.; Shinde, D. B. Mendeleev Commun. 2005, 15, 150.

389. Tajbakhsh, M.; Mohajerani, B.; Heravi, M. M.; Ahmadi, A. N. J. Mol. Catal. A. Chem. 2005, 236, 216.

390. Cepanec, I.; Litvic, M.; Bartolincic, L. M. Tetrahedron 2005, 61, 4275. 
391. Su, W.; Li, J.; Zheng, Z.; Shen, Y. Tetrahedron Lett. 2005, 46, 6037.

392. Heravi, M. M.; Derikvand, F.; Bamoharram, F. F. J. Mol. Catal. A. Chem. 2005, 242, 173.

393. Saxena, I.; Borah, D. C.; Sarma, J. C. Tetrahedron Lett. 2005, 46, 1159.

394. Sabitha, G.; Reddy, K. B.; Yadav, J. S.; Shailaja, D.; Sivudu, K. S. Tetrahedron Lett. 2005, 46, 8221.

395. El-Badaoui, H.; Bazi, F.; Tahir, R.; Lazrek, H. B.; Sebti, S. Catal. Commun. 2005, 6, 455.

396. Wang, H. S.; Zhao, L. F.; Miao, J. Y. Chem. Reagents 2005, 27, 171.

397. Li, M.; Guo, W. S.; Wen, L. R.; Zhang, X. L. Chin. J. Org. Chem. 2005, 25, 1062.

398. Zhang, Y. Q.; Wang, C.; Li, G. S.; Li, J. C.; Liu, H. M.; Wu, Q. H, Chin. J. Org. Chem. 2005, 25, 1265.

399. El Badaoui, H.; Bazi, F.; Sokori, S.; Boulaajaj, S.; Lazrek, H. B.; Sebti, S. Lett. Org. Chem. 2005, 2, 561.

400. Ding, X.; Shi, L.; Jing, X. Adv. Fine Petro. Chem. 2005, 6, 26.

401. Luis, M. T.; Frija, I. V.; Khmelinskii, M. L. S. C. Tetrahedron Lett. 2005, 46, 6757.

402. Legeay, J-C.; Vanden Eynde, J. J.; Bazureau, J. P. Tetrahedron 2005, 61, 12386.

403. Khodaei, M. M.; Khosropour, A. R.; Bigzadeh, M. Synth. Commun. 2004, 34, 1551.

404. Bose, A. K.; Pednekar, S.; Ganguly, S. N.; Chakraborty, G.; Manhas, M. S. Tetrahedron. Lett. 2004, 45, 8351.

405. Shaabani, A.; Bazgir, A. Tetrahedron Lett. 2004, 45, 2575.

406. Martins, M. A. P.; Teixeira, M. V. M.; Cunico, W.; Scapin, E.; Mayer, R.; Pereira, C. M. P.; Zanatta, N.; Bonacorso, H. G.; Peppe, C.; Yuan, Y-F. Tetrahedron Lett. 2004, 45, 8991.

407. Lee, K.Y.; Ko, K.Y. Bull. Korean Chem. Soc. 2004, 25, 1929.

408. Yadav, J. S.; Reddy, B. V. S.; Naidu, J. J.; Sadashiv, K. Chem. Lett. 2004, 33, 926.

409. Yadav, J. S.; Kumar, S. P.; Kondaji, G.; Rao, R. S.; Nagaiah, K. Chem. Lett. 2004, 33, 1168.

410. Hua, G. P.; Tu, S. J.; Fang, F.; Tu, M. S.; Shi, D. Q.; Wang, X. S. Chin. J. Struct. Chem. 2004, 23, 1295.

411. Zhu, Y.; Pan, Y.; Huang, S. Synth. Commun. 2004, 34, 3167.

412. Yadav, J. S.; Subba Reddy, B. V.; Sridhar, P.; Reddy, J. S. S.; Nagaiah, K.; Lingaiah, N.; Saiprasad, P. S. Eur. J. Org. Chem. 2004, 552.

413. Sun, Q.; Wang, Y.; Ge, Z.; Cheng, T.; Li, R. Synthesis 2004, 1047.

414. Bahekar, S. S.; Kotharkar, S. A.; Shinde, D. B. Mendeleev Commun. 2004, 14, 210.

415. Gholap, A. R.; Venkatesan, K.; Daniel, T.; Lahoti, R. J.; Srinivasan, K. V. Green. Chem. 2004, 6, 147.

416. Xu, L. W.; Wang, Z. T.; Xia, C. G.; Li, L.; Zhao, P. Q. Helv. Chim. Acta 2004, 87, 2608.

417. Russowsky, D.; Lopes, F. A.; da Silva, V. S. S.; Canto, K. F. S.; Montes Doca, G. M.; Godoi, M. N. J. Braz. Chem. Soc. 2004, 15, 165.

418. Kumar, S.; Saini, A.; Sandhu, J. S. Indian J. Chem. 2004, 43B, 1485. 
419. Shanmugam, P.; Sabastein, C.; Perumal, P. T. Indian J. Chem. 2004, 43B, 135.

420. Misra, A. K.; Agnihotri, G.; Madhusudan, S. K. Indian J. Chem. 2004, 43B, 2018.

421. Shaabani, A.; Bazgir, A.; Arab-Ameri, S. Phosphorus Sulfur Silicon Relat. Elem. 2004, $179,2169$.

422. Salehi, H.; Guo, Q. X. Synth. Commun. 2004, 34, 171.

423. Tu, S.; Fang, F.; Zhu, S.; Li, T.; Zhang, X.; Zhuang, Q. Synlett. 2004, 537.

424. Tu, S.; Fang, F.; Zhu, S.; Li, T.; Zhang, X.; Zhuang, Q. J. Heterocycl. Chem. 2004, 41, 253.

425. Kidwai, M.; Bala, S.; Mishra, A. D. Indian J. Chem. 2004, 43B, 2485.

426. Gangadasu, B.; Palaniappan, S.; Rao, V. J. Synlett 2004, 1285.

427. Hazarkhani, H.; Karimi, B. Synthesis 2004, 1239.

428. Venkat Narsaiah, A.; Basak, A. K.; Nagaiah, K. Synthesis 2004, 1253.

429. Srinivas, K. V. N. S.; Das, B. Synthesis 2004, 2091.

430. Bhosale, R. S.; Bhosale, S. V.; Bhosale, S. V.; Wang, T.; Zubaidha, P. K. Tetrahedron Lett. 2004, 45, 9111.

431. Khodaei, M. M.; Khosropour, A. R.; Bigzadeh, M. Synth. Commun. 2004, 34, 1551.

432. Shailaja, M.; Manjula, A.; Vittal Rao, B.; Parvathi, N. Synth. Commun. 2004, 34, 1559.

433. Khodaei, M. M.; Salehi, P.; Zolfigol, M. A.; Sirouszadeh, S. Polish J. Chem. 2004, 78, 385.

434. Reddy, Y. T.; Rajitha, B.; Reddy, P. N.; Kumar, B. S.; Rao, V. P. G Synth. Commun. 2004, 34, 3821 .

435. Jin, T. S.; Xiao, J. C.; Chen, Y. X.; Li, T. S. J. Chem. Res. Synop. 2004, 3, 190.

436. Gohain, M.; Prajapati, D.; Sandhu, J. S. Synlett 2004, 235.

437. Adharvana Chari, M.; Syamasundar, K. J. Mol. Catal. A. Chem. 2004, 221, 137.

438. Jenner, G. Tetrahedron Lett. 2004, 45, 6195.

439. Subhas Bose, D.; Kumar, R. K.; Fatima, L. Synlett 2004, 279.

440. Ghosh, R.; Maiti, S.; Chakraborty, A. J. Mol. Catal. A. Chem. 2004, 217, 47.

441. Yarapathi, R. V.; Kurva, S.; Tammishetti, S. Catal. Commun. 2004, 5, 51.

442. Chen, W-Y.; Lu, J. Chin. J. Org. Chem. 2004, 24, 1111.

443. Shao, G. Q. Chin. J. Synth. Chem. 2004, 12, 325.

444. Wang, M.; Jiang, H.; Wang, R.; Wang, Z. J. Chin. Rare Earth Soc. 2004, 22, 81.

445. Mobinikhaledi, A.; Foroughifar, N.; Fathinejad Jirandehi, H.; Phosphorus Sulfur Silicon Relat. Elem. 2004, 179, 2259.

446. Lusch, M. J.; Tallarico, J. A. Org. Lett. 2004, 6, 3237.

447. vanden Eynde, J. J.; Watte, O. Arkivoc 2003, (iv), 93.

448. Sabitha, G.; Reddy, G. S. K. K.; Reddy, K. B.; Yadav, J. S. Tetrahedron Lett. 2003, 44, 6497.

449. Osnaya, R.; Arroyo, G. A.; Parada, L.; Delgado, F.; Trujillo, J.; Salmon, M.; Miranda, R. Arkivoc 2003, (xi), 112. 
450. Stadler, A.; Yousefi, B. H.; Dallinger, D.; Walla, P.; Van der Eycken, E.; Kaval, N.; Kappe, C. O. Org. Proc. Res. Dev. 2003, 7, 707.

451. Maiti, G.; Kundu, P.; Guin, C. Tetrahedron Lett. 2003, 44, 2757.

452. Li, J. T.; Han, J. F.; Yang, J. H.; Li, T. S. Ultrason. Sonochem. 2003, 10, 119.

453. Salehi, P.; Dabiri, M.; Zolfigol, M. A.; Bodaghi Fard, M. A. Heterocycles 2003, 60, 2435.

454. Salehi, P.; Dabiri, M.; Zolfigol, M. A.; Bodaghi Fard, M. A. Tetrahedron Lett. 2003, 44, 2889.

455. Varala, R.; Mujahid Alam, M.; Adapa, S. R. Synlett 2003, 67.

456. Mitra, A. K.; Banerjee, K. Synlett 2003, 1509.

457. Sabitha, G.; Reddy, G. S. K. K.; Reddy, C. S.; Yadav, J. S. Synlett 2003, 858.

458. Xia, M.; Wang, Y. Synthesis 2003, 262.

459. Gong, D.; Zhang, L.; Yuan, C. Heteroatom. Chem. 2003, 14, 13.

460. Wang, L.; Qian, C.; Tian, H.; Yun, M. A. Synth. Commun. 2003, 33, 1459.

461. Dondoni, A.; Massi, A.; Minghini, E.; Sabbatini, S.; Bertolasi, V. J. Org. Chem. 2003, $68,6172$.

462. Munoz-Muniz, O.; Juaristi, E. Arkivoc 2003, (xi), 16.

463. Shanmugam, P.; Annie, G.; Perumal, P. T. J. Heterocycl. Chem. 2003, 40, 879.

464. Kidwai, M.; Mohan, R.; Saxena, S. Russ. Chem. Bull. Int. Ed. 2003, 52, 2457.

465. Tu, S. J.; Fang, F.; Miao, C. B.; Jiang, H.; Shi, D. Q. Chin. J. Chem. 2003, 21, 706.

466. Xu, H.; Wang, Y. G. Chin. J. Chem. 2003, 21, 327.

467. Li, Y. X.; Bao, W. L. Chin. Chem. Lett. 2003, 14, 993.

468. Foroughifar, N.; Mobinikhaledi, A.; Fathinejad Jirandehi, H. Phosphorus Sulfur Silicon Relat. Elem. 2003, 178, 1241.

469. Foroughifar, N.; Mobinikhaledi, A.; Fathinejad Jirandehi, H.; Memar, S. Phosphorus Sulfur Silicon Relat. Elem. 2003, 178, 1269.

470. Foroughifar, N.; Mobinikhaledi, A.; Fathinejad Jirandehi, H. Phosphorus SulfurSilicon Relat. Elem. 2003, 178, 495.

471. Bose, D. S.; Fatima, L.; Mereyala, H. B. J. Org. Chem. 2003, 68, 587.

472. Fu, N. Y.; Yuan, Y. F.; Pang, M. L.; Wang, J. T.; Peppe, C. J. Organomet. Chem. 2003, 672, 52.

473. Paraskar, A. S.; Dewkar, G. K.; Sudalai, A. Tetrahedron Lett. 2003, 44, 3305.

474. Reddy, K. R.; Reddy, C. V.; Mahesh, M.; Raju, P. V. K.; Reddy, V. V. N. Tetrahedron Lett. 2003, 44, 8173.

475. Shaabani, A.; Bazgir, A.; Teimouri, F.Tetrahedron Lett. 2003, 44, 857.

476. Martinez, S.; Meseguer, M.; Casas, L.; Rodríguez, E.; Molins, E.; Moreno-Manas, M.; Roig, A.; Sabastian, R. M.; Vallribera, A. Tetrahedron. 2003, 59, 1553.

477. Choudhary, V. R.; Tillu, V. H.; Narkhede, V. S.; Borate, H. B.; Wakharkar, R. D. Catal. Commun. 2003, 4, 449.

478. Jin, T. S.; Xiao, J. C.; Li, T. S. Chin. J. Org. Chem. 2003, 23, 93. 
479. Fu, N. Y.; Pang, M. L.; Yu, Y. F.;Wang, J. T. Chem. J. Chin. Univ 2003, 24, 79.

480. Shanmugam, P.; Perumal, P. T. J. Chem. Res. 2003, 601.

481. Reddy, K. R.; Reddy, C. V.; Mahesh, M.; Raju, P.V. K.; Reddy, V.V. N. Tetrahedron Lett. 2003, 44, 8173.

482. Tu, S.; Fang, F.; Miao, C.; Jiang, H.; Feng, Y.; Shi, D.; Wang, X. Tetrahedron Lett. 2003, $44,6153$.

483. Jin, T.; Zhang, S.; Li, T. Synth. Commun. 2002, 32, 1847.

484. Baruah, P. P.; Gadhwal, S.; Prajapati, D.; Sandhu, J. S. Chem. Lett. 2002, 31, 1038.

485. Lu, J.; Bai, Y. Synthesis 2002, 466.

486. Dondoni, A.; Massi, A.; Sabatini, S. Tetrahedron Lett. 2002, 43, 5913.

487. Tu, S. J.; Zhou, J. F.; Cai, P. J.; Wang, H.; Feng, J. C. Synth. Commun. 2002 , 32, 147.

488. Fu, N. Y.; Yuan, Y. F.; Cao, Z.; Wang, S. W.; Wang, J. T.; Peppe, C. Tetrahedron 2002, $58,4801$.

489. Venkateshwar Reddy, C.; Mahesh, M.; Raju, P. V. K.; Ramesh Babu, T.; Narayana Reddy, V. V. Tetrahedron Lett. 2002, 43, 2657.

490. Peng, J.; Deng, Y. Chin. J. Org. Chem. 2002, 22, 71.

491. Lu, J.; Bai, Y. J.; Guo, Y. H.; Wang, Z. J.; Ma, H. R. Chin. J. Chem. 2002, 20, 681.

492. Xue, S.; Shen, Y. C.; Li, Y. L.; Shen, X. M.; Guo, Q. X. Chin. J. Chem. 2002, 20, 385.

493. Chen, R. F.; Qian, C. T. Chin. J. Chem. 2002, 20, 427.

494. Lu, J.; Wang, F.; Bai, Y.; Li, W. Chin. J. Org. Chem. 2002, 22, 788.

495. Fu, N. Y.; Pang, M. L.; Yuan, Y. F.; Wang, J. T. Chin. Chem. Lett. 2002, 13, 921.

496. Kidwai, M.; Saxena, S.; Mohan, R.; Venkataramanan, R. J. Chem. Soc. Perkin Trans. 1 2002, $1,1845$.

497. Xia, M.; Wang, Y-G. Tetrahedron Lett. 2002, 43, 7703.

498. (a) Anastas, P. T.; Warner, J. C. Green Chemistry: Theory and Practice; Oxford University Press: New York, 1998, p 30. (b) Suresh; Sandhu, J. S. Recent Advances in Ionic Liquids: Green Unconventional Solvents of This Century Part-I, Green Chem. Lett. Rev. 2011, in press (c) Suresh; Sandhu, J. S. Recent Advances in Ionic Liquids : Green Unconventional Solvents of This Century Part-II, Green Chem. Lett. Rev. 2011, in press.

499. Peng, J.; Deng, Y. Tetrahedron Lett. 2001, 42, 5917.

500. Schnell, B.; Krenn, W.; Faber, K.; Kappe, C. O. J. Chem. Soc., Perkin Trans.1. 2001, 4382.

501. Gedye, R.; Smith, F.; Westaway, K.; Ali, H.; Baldisera, L.; Laberge, L.; Rousell, J. Tetrahedron Lett. 1986, 27, 279.

502. Gupta, R.; Gupta, A. K.; Paul, S.; Kachroo, P. L. Indian J. Chem. 1995, 34B, 151.

503. (a) Studer, A.; Hadida, S.; Ferritto, R.; Kim, S. Y.; Jeger, P.; Wipf, P.; Curram, D. P. Science 1997, 275, 823. (b) Studer, A.; Jeger, P.; Wipf, P.; Curram, D. P. J. Org. Chem. 1997, 62, 2917. (c) Valverde, M. G.; Dallinger, D.; Kappe, C. O. Synlett 2001, 741.

504. (a) Dondoni, A.; Massi, A. Tetrahedron Lett. 2001, 42, 7975. (b) Kappe, C. O. Bioorg. Med. Chem. Lett. 2000, 10, 49. 
505. (a) Bhuyan, P. J.; Borah, R. C.; Sandhu, J. S. J. Org. Chem. 1990, 55, 568. (b) Bhuyan, P. J.; Sandhu, J. S.; Ghosh, A. C. Tetrahedron Lett. 1996, 37, 1853. (c) Bhuyan, P. J.; Lekhok, K. C.; Sandhu, J. S. Tetrahedron Lett. 1999, 40, 1793. (d) Bhuyan, P. J.; Borah, H. N.; Sandhu, J. S. J. Chem. Soc., Perkin Trans.1. 1999, 3083. (e) Bhuyan, P. J.; Borah, H. N.; Sandhu, J. S. Tetrahedron Lett. 2002, 43, 895. (f) Noguchi, M.; Kajigaeshi, N. S. Chem. Pharm. Bull. 1986, 34, 3994. (g) Koroniak, H.; Karwatka, P.; Cytlak, T. Tetrahedron Lett. 2004, 45, 5767.

506. G. C.; Nandi, S.; Samai, M. S. Singh, J. Org. Chem. 2010, 75, 7785.

507. (a) Saini, A.; Kumar, S.; Sandhu, J. S. Indian J. Chem. 2006, 45B, 684. (b) Wang, Z-T.; Xu, L-W.; Xia, C -G.; Wang, H-Q. Tetrahedron Lett. 2004, 45, 7951. (c) Pandit, S.; Shaikh, R.; Pandit, V. Rasayan J. Chem. 2009, 2, 907.

508. Zhu, Y.; Huang, S.; Wan, J.; Yan, L.; Pan, Y.; Wu, A. Org. Lett. 2006, 8, 2599.

509. Chebanov, V. A.; Saraev, V. E.; Desenko, S. M.; Chernenko, V. N.; Knyazeva, I. V.; Groth, U.; Glasnov, T. N.; Kappe, C. O. J. Org. Chem. 2008, 73, 5110.

510. Dondoni, A.; Massi, A.; Sabbatini, S.; Bertolasi, V. J. Org. Chem. 2002, 67, 6979.

511. Prakash, O.; Pannu, K.; Naithani, R.; Kaur, H. Synth. Commun. 2006, 36, 3479.

512. Wang, C.; Xu, H.; Xie, Z.; Wang, X.; Zhang, Z.; Sun, Q. Steroids 2010, 75, 1033.

513. Gladkov, E.; Sirko, S.; Khanetskii, B.; Lukinova, E.; Desenko, S. Chem. Pap. 2007, 61, 146.

514. Quan, Z-J.; Ren, R-G.; Jia, X-D.; Da, Y-X.; Zhang, Z.; Wang, X-C. Tetrahedron 2011, $67,2462$.

515. Wang, X.; Quan, Z.; Wang, J-K.; Zhanga, Z.; Wang, M. Bioorg. Med. Chem. Lett. 2006, 16, 4592.

516. Azizian, J.; Mirza, B.; Mojtahedi, M. M.; Abaee, M. S.; Sargordan, M. J. Fluor. Chem. 2008, 129, 1083.

517. Mamedov, V. A.; Vdovina, S. V.; Vorkunova, E. I.; Mustakimova, L. V.; Saifina, A. F.; Gubaidullin, A. T.; Rizvanov, I. K.; Levin, Y-A.; Litvinov, I. A. Russ. Chem. Bull. Int. Ed. 2008, 57, 1257.

518. Shaabani, A.; Bazgir, A.; Bijanzadeh, H. R. Mol. Divers. 2004, 8, 141.

519. (a) Byk, G.; Gottlieb, H. E.; Herscovici, J.; Mirkin, F. J. Comb. Chem. 2000, 2, 732. (b) Byk, G.; Kabha, E. J. Comb. Chem. 2004, 6, 596.

520. Fustero, S.; Catalan, S.; Piera, J.; Sanz-Cervera, J. F.; Fernandez, B.; Acena, J. L. J. Org. Chem. 2006, 71, 4010.

521. Sharma, P.; Kumar, A.; Rane, N.; Gurram, V. Tetrahedron 2005, 61, 4237.

522. Singh, K.; Singh, S.; Mahajan, A. J. Org. Chem. 2005, 70, 6114.

523. (a) Lavilla, R. J. Chem. Soc., Perkin Trans. 1 2002, 1142. (b) Khanina, E. L.; Duburs, G. Khim. Geterotsikl. Soedin. 1982, 535. (c) Akhtar, M. S.; Seth, M.; Bhaduri, A. P. Indian J. Chem. 1987, 26B, 556. (d) Khanina, E. L.; Liepinsh, E. E.; Mutsenietse, E. K.; Duburs, G. Khim. Geterotsikl. Soedin. 1987, 668. (e) Slavinskaya, V. A.; Duburs, G.; Sile, D.; Kreile, D.; Khanina, E. L. U.S.S.R. Patent 632,695. 1978. Chem. Abstr. 1979, 90, 
121631y. (f) Bagley, M. C.; Lubinu, M. C. Synthesis 2006, 8, 1283. (g) Han, B.; Liu, Z.; Liu, Q.; Yang, L.; Liu, Z. L.; Yu, W. Tetrahedron 2006, 62, 2492. (h) Anniyappan, M.; Muralidharan, D.; Perumal, P. T. Tetrahedron 2002, 58, 5069. (i) Nakamichi, N.; Kawashita, Y.; Hayashi, M. Org. Lett. 2002, 4, 3955. (j) Eynde, J. J. V.; Audiart, N.; Canonne, V.; Michel, S.; Haverbeke, Y. V.; Kappe, C. O. Heterocycles 1997, 45, 1967. (k) Jin, M-Z.; Yang, L.; Wu, L-M.; Liu, Y-C.; Liu, Z-L. Chem. Commun. 1998, 2451. (1) Zhu, X-Q.; Zhao, B-J.; Cheng, J-P. J. Org. Chem. 2000, 65, 8158. (m) Zhang, D.; Wu, LZ.; Zhou, L.; Han, X.; Yang, Q. Z.; Zhang, L. P.; Tung, C. H. J. Am. Chem. Soc. 2004, 126, 3440. (n) Fang, X.; Liu, Y-C.; Li, C. J. Org. Chem. 2007, 72, 8608. (o) Atwal, K. S.; Rovnyak, G. C.; Kimball, S. D.; Floyd, D. M.; Moreland, S.; Swanson, B. N.; Gougoutas, J. Z.; Schwartz, J.; Smillie, K. M.; Malley, M. F. J. Med. Chem. 1990, 33, 2629.

524. Watanabe, M.; Koike, H.; Ishiba, T.; Okada, T.; Seo, S.; Hirai, K. Bioorg. Med. Chem. 1997, 5, 437.

525. Puchala, A.; Belaj, F.; Bergman, J.; Kappe, C. O. J. Het. Chem. 2001, 38, 1345.

526. Yamamoto, K.; Chen, Y. G.; Buono, F. G. Org. Lett. 2005, 7, 4673.

527. Memarian, H. R.; Farhadi, A. Ultrason. Sonochem. 2008, 15, 1015.

528. Singh, K.; Singh, K. Aust. J. Chem. 2008, 61, 910.

529. Liang, R. R.; Wu, G. L.; Wu, W. T.; Wu, L. M. Chin. Chem. Lett. 2009, 20, 1183.

530. Memarian, H. R.; Soleymani, M. Ultrason. Sonochem. 2011, 18, 745.

531. Liu, Q.; Li, Y-N.; Zhang, H-H.; Chen, B.; Tung, C-H.; Wu, L-Z. J. Org. Chem. 2011, 76, 1444.

532. (a) Atwal, K. S.; Swanson, B. N.; Unger, S. E.; Floyd, D. M.; Moreland, S.; Hedberg, A.; O'Reilly, B. C. J. Med. Chem. 1991, 34, 806. (b) Rovnyak, G. C.; Atwal, K. S.; Hedberg, A.; Kimball, S. D.; Moreland, S.; Gougoutas, J. Z.; O’Reilly, B. C.; Schwartz, J.; Malley, M. F. J. Med. Chem. 1992, 35, 3254. (c) Grover, G. J.; Dzwonczyk, S.; McMullen, D. M.; Normandin, D. E.; Parham, C. S.; Sleph, P. G.; Moreland, S. J. Cardiovasc. Pharmacol. 1995, 26, 289. (d) Rovnyak, G. C.; Kimball, S. D.; Beyer, B.; Cucinotta, G.; DiMarco, J. D.; Gougoutas, J.; Hedberg, A.; Malley, M.; McCarthy, J. P.; Zhang, R.; Moreland, S. J. Med. Chem. 1995, 38, 119.

533. Gong, L. Z.; Chen, X. H.; Xu, X. Y. Chem.-Eur. J. 2007, 13, 8920.

534. Atwal, K. S.; Swanson, B. N.; Unger, S. E.; Floyd, D. M.; Moreland, S.; Hedberg, A.; O'Reilly, B. C. J. Med. Chem. 1991, 34, 806.

535. (a) Maliga, Z.; Kapoor, T. M.; Mitchison, T. J. Chem. Biol. 2002, 9, 989. (b) DeBonis, S.; Simorre, J. P.; Crevel, I.; Lebeau, L.; Skoufias, D. A.; Blangy, A.; Ebel, C.; Gans, P.; Cross, R.; Hackney, D. D.; Wade, R. H.; Kozielski, F. Biochemistry 2003, 42, 338.

536. Olszewski, A.; Weiss, G. A. J. Am. Chem. Soc. 2005, 127, 12178.

537. Blasco, M. A.; Thumann, S.; Wittmann, J.; Giannis, A.; Groger, H. Bioorg. Med. Chem. Lett. 2010, 20, 4679.

538. (a) Dondoni, A.; Massi, A.; Sabbatini, S.; Bertolasi, V. J. Org. Chem. 2002, 67, 6979. (b) Dondoni, A.; Massi, A.; Sabbatini, S. Tetrahedron Lett. 2002, 43, 5913. 
539. Huang, Y.; Yang, F.; Zhu, C. J. Am. Chem. Soc. 2005, 127, 16386.

540. (a) Chen, X-H.; Xu, X-Y.; Liu, H.; Cun, L-F.; Gong, L-Z. J. Am. Chem. Soc. 2006, 128, 14802. (b) Li, N.; Chen, X-H.; Song, J.; Luo, S-W.; Fan, W.; Gong, L-Z. J. Am. Chem. Soc. 2009, 131, 15301.

541. (a) Wu, Y-Y.; Chai, Z; Liu, X-Y.; Zhao, G.; Wang, S-W. Eur. J. Org. Chem. 2009, 6, 904. (b) Kappe, C. O. Acc. Chem. Res. 2000, 33, 879. (c) Saha, S.; Moorthy, J. N. J. Org. Chem. 2011, 76, 396. (d) Xin, J.; Chang, L.; Hou, Z.; Shang, D.; Liu, X.; Feng, X. Chem. Eur. J. 2008, 14, 3177. (e) Wang, Y.; Yu, J.; Miao, Z.; Chen, R. Org. Biomol. Chem. 2011, 9, 3050. (f) Olvera, R. D.; Demare, P.; Regla, I.; Juaristi, E. Arkivoc 2008, (vi), 61. (g) Singh, K.; Singh, S. Tetrahedron 2009, 65, 4106. (h) Goss, J. M.; Schaus, S. E. J. Org. Chem. 2008, 73, 7651.

542. Wan, J-P.; Wang, C.; Pan, Y. Tetrahedron 2011, 67, 922.

\section{Authors' Biographies}

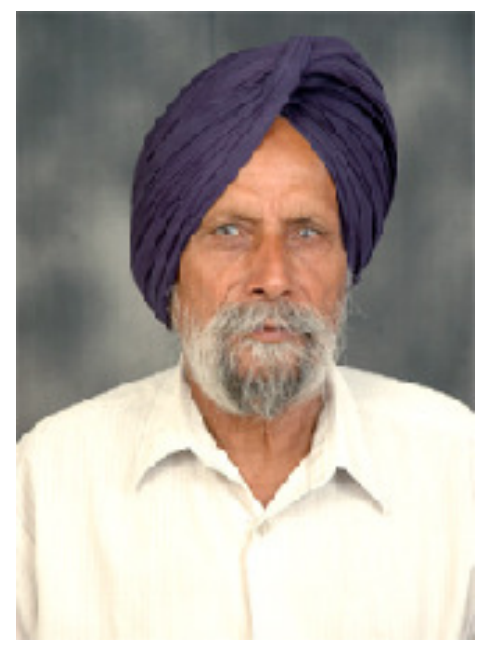

Jagir S. Sandhu was born in 1942 in Amritsar, Punjab. He is a senior scientist of Indian National Science Academy (INSA), New Delhi. He received his B.Sc in 1962 from Punjab University and subsequently got M.Sc. in 1965 and has awarded his Ph.D. degree in chemistry in 1969 from Punjabi University Patiala. He did his post doctoral work at Indiana University Bloomington, Indiana in USA with Prof. E. Compaign in the area of synthetic organic chemistry. In 1976, Dr. Sandhu joined regional research laboratory (RRL), Jorhat (CSIR) as Head of Medicinal Chemistry Division and in stages he arose to the position of Director, RRL Jorhat, Assam from where he retired in 2002. He established the chemistry of pharmacologically significant privileged molecules based on Uracil, Benzopyrans, other related $\mathrm{N} \& \mathrm{O}$ 
Heterocycles and also contribute to green chemistry namely functional group transformations based on catalysts developments, including enzymes, ionic liquids etc. using green techniques like ultrasound, microwave irradiation, grinding etc. as a result of these researches $27 \mathrm{Ph} . \mathrm{D}$ students received their degrees and are established in academic and industry institutions and abroad also. His broad area of research is heterocyclic chemistry, cycloadditions 1-3 (1,3 dipolar), 1-4, inter \& intera molecular ones. He has published 255 research papers in reputed journals, apart from some, patents as well as reviews and Books. He is life member of several learnt bodies of this country like, chemical society, chemical research society of India and is Fellow of National Academy of science Allahabad (FNASc) and is also fellow of Indian Science Academy (FNA), New Delhi.

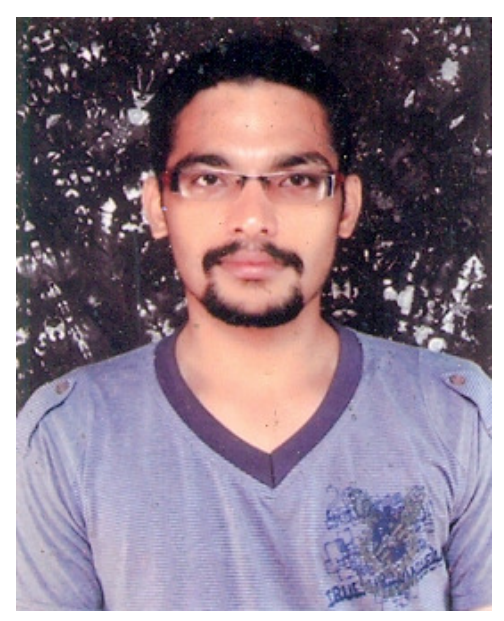

Suresh is a research associate of organic chemistry at Punjabi University, Patiala, Punjab. He was born in 1983 in Jind, Haryana and received both B.Sc degree (2003) and M.Sc degree (2005) form Kurukshetra University, Kurukshetra. He is senior research fellow (SRF) on Council of Science and Industrial Research (CSIR) major research project and pursuing Ph.D degree under the supervision of Dr. Jagir S. Sandhu, FNA, INSA Sr. Scientist from 2007 onwards. His research interests are in heterocycles based on pyrimidines, related heterocycles and functional group transformations mainly focused to green chemistry. 